Série des Documents de Travail

$n^{\circ}$ 2013-24

The Implementation
of Stabilization Policy

O. LOISEL ${ }^{1}$

December, 2013

Les documents de travail ne reflètent pas la position du CREST et n'engagent que leurs auteurs.

Working papers do not reflect the position of CREST but only the views of the authors.

1 CREST (ENSAE). Address : CREST, 15 Boulevard Gabriel Péri, 92245 Malakoff Cedex, France. Email : olivier.loisel@ensae.fr 


\title{
The Implementation of Stabilization Policy
}

\author{
Olivier Loisel*
}

3 December 2013

\begin{abstract}
This paper addresses the issue of what path for the economy a policymaker can implement as the unique local equilibrium, given her observation set. I consider a broad class of locally linearizable dynamic stochastic discrete-time infinite-horizon rational-expectations models, which includes most existing DSGE models. I consider various alternative observation sets for the policymaker, depending on what she observes of the private sector's actions, its expectations, and exogenous shocks, and allowing for observation lags of any length. For each observation set and any local path that is consistent with the structural equations and this observation set, I provide conditions for the existence of a policyinstrument rule that is consistent with this observation set and implements that path as the unique local equilibrium. I also show how to design this rule arithmetically, and thus obtain its coefficients as rational functions of the parameters of the model and path considered. I apply these results to, and derive some of their implications for, the implementation of optimal monetary policy, monetary policy based on constant-interest-rate forecasts, debt-stabilizing tax policy, and optimal tax policy.
\end{abstract}

Keywords: dynamic stochastic rational-expectations models, stabilization policy, local equilibrium determinacy, local path implementation.

JEL codes: E52, E61.

*CREST (ENSAE), Malakoff, France. Address: CREST, 15 boulevard Gabriel Péri, 92245 Malakoff Cédex, France. Email address: olivier.loisel@ensae.fr. I am grateful to Gadi Barlevy, Fabio Canova, Roger Farmer, Marc Giannoni, Jesper Lindé, Patrick Pintus, Carl Walsh, Tao Zha, colleagues at CREST, and especially Behzad Diba, Christian Hellwig, and Michael Woodford, for useful comments at various stages of this long project. The usual disclaimer applies. 


\section{Introduction}

It is common practice nowadays to model macroeconomic stabilization policy by a policy-instrument rule ensuring local equilibrium determinacy, i.e. local equilibrium existence and uniqueness, in a locally linearizable dynamic stochastic discrete-time infinite-horizon rational-expectations model. For instance, monetary policy is routinely modeled as an interest-rate rule ensuring local equilibrium determinacy in a dynamic stochastic general-equilibrium (DSGE) model. By ensuring local equilibrium determinacy, such a rule enables the policymaker to preclude the kind of macroeconomic fluctuations that, according to Clarida, Galí, and Gertler (2000), may have occurred in the U.S. before 1979.

Despite the widespread nature of this practice, very little is known about what paths for the economy the policymaker can implement as the unique local equilibrium with such rules, given her observation set. The goal of this paper is to address this question in a broad class of locally linearizable dynamic stochastic discrete-time infinite-horizon rational-expectations models, which includes most existing DSGE models. For any policymaker's observation set, I define a feasible path as a local path that is consistent with the structural equations and the policymaker's observation set, and an implementable path as a feasible path that can be implemented as the unique local equilibrium by a policy-instrument rule that is consistent, both in and out of equilibrium, with the policymaker's observation set. The contribution of the paper is essentially threefold. First, I (partially) characterize the subset of implementable paths, within the set of feasible paths, for various alternative policymaker's observation sets. Second, for each implementable path, I show how to design a corresponding policy-instrument rule arithmetically, i.e. with a finite number of arithmetic operations (addition, subtraction, multiplication, and division), and thus obtain its coefficients as rational functions of the structural and implementable-path parameters. Third, I apply these general results to, and derive their implications for, the implementation of some specific stabilization policies, namely optimal monetary policy, monetary policy based on constant-interest-rate forecasts, debt-stabilizing tax policy, and optimal tax policy, in some specific models.

The observation sets that I consider for the policymaker may include four kinds of variables: her own past actions (i.e., past policy instruments), the private sector's past actions, its past expectations, and current and past exogenous shocks. ${ }^{1}$ The private sector's actions should be interpreted in a broad sense: in most applications, they will typically include both quantities and prices. The private sector's expectations are its expectations of its future actions or the future policy instrument. ${ }^{2}$ I argue in the paper that the timing assumption that is best suited to the study of stabilization-policy implementation requires the policymaker to play before the private sector at each date. Accordingly, none of the policymaker's observation sets that I consider includes any private sector's current action or expectation, and none of the policy-instrument rules that I design involve any private sector's current action or expectation either. In the terminology first used by Svensson (1999), these rules are explicit.

It is well known, since Sargent and Wallace (1975), that when the policymaker only observes exogenous shocks and her own actions, no feasible path may be implementable. I show that, in this case, adding the

\footnotetext{
${ }^{1}$ Examples of observable exogenous shocks include exogenous policy measures, foreign developments (considered as exogenous from the point of view of a small open economy), technological inventions, weather conditions, and natural disasters.

${ }^{2}$ Admittedly, the private sector's expectations are thoughts and, unless she manages to monitor and correctly interpret private agents' brain activity, the policymaker cannot directly observe them. Instead, she may be able to infer them from her observation of some private sector's actions, like financial-market prices and survey responses. For simplicity and without any loss in generality, I abstract from these financial markets and surveys, and proceed as if she could directly observe those expectations.
} 
private sector's actions to her observation set - however long the lag with which she observes them overturns the result and makes all feasible paths implementable. ${ }^{3}$ I also show that, under a fairly weak condition, all feasible paths are implementable when the policymaker observes actions and expectations, again possibly with lags, but not exogenous shocks. Finally, I show that, when the policymaker observes only actions, again possibly with lags, there exist some feasible paths that are not implementable, even when there is one more action in her observation set than exogenous shocks in the model. In particular, this can be the case of the path that is Ramsey-optimal from Woodford's (1999a) "timeless perspective" in Svensson and Woodford's (2005) New Keynesian model with policy transmission lags for empirically relevant values of its structural parameters.

Most of the policy-instrument rules encountered in the literature, at least in the context of DSGE models of the monetary transmission mechanism, manage to ensure local equilibrium determinacy by requiring the policy instrument to react out of equilibrium to the private sector's current actions or expectations. ${ }^{4}$ By preventing the policymaker from reacting out of equilibrium to these current actions or expectations, my timing assumption thus puts her behind the curve. Policy implementation lags (which compel her to choose unconditionally in advance the value of her policy instrument, as in Schmitt-Grohé and Uribe, 1997) or observation lags, when present, put her still further behind the curve by preventing her from reacting out of equilibrium to the private sector's recent actions or expectations. I find nonetheless that, in many cases, neither this timing assumption nor those policy implementation or observation lags reduce the policymaker's ability to control the economy, in the sense that they do not prevent any feasible path from being implementable. In essence, this result comes from the fact that, in models that raise non-trivial local-equilibrium-indeterminacy issues, the private sector's current actions depend directly on its expectation of its future actions and, therefore, indirectly on its expectation of the future policy instrument. Now, by imposing an out-of-equilibrium reaction of the current policy instrument to the private sector's past actions, a policy-instrument rule also imposes an out-of-equilibrium reaction of the private sector's expectation of the future policy instrument to its current actions. The latter reaction can be viewed as the feedback mechanism that enables the policymaker to control the economy.

On the methodological front, for each implementable path, I show how to design a corresponding policyinstrument rule arithmetically, i.e. with a finite number of arithmetic operations (addition, subtraction, multiplication, and division). This method of designing policy-instrument rules does not require, in particular, the determination of any polynomial roots, nor equivalently the determination of any matrix eigenvalues. Therefore, it does not require the use of any existing algorithm to solve locally linearizable dynamic stochastic discrete-time infinite-horizon rational-expectations models (e.g., the algorithms developed by Klein, 2000, and Sims, 2002). Instead, it uses Bézout's identity, the Euclidean division, and Cramer's rule, all of which involve a finite number of arithmetic operations, to directly transform the polynomials characterizing the structural equations and the implementable path considered into the polynomials characterizing the policy-instrument rule. This arithmetic-designability property implies that, for each model, policy instrument, observation set, and implementable path, the coefficients of the policy-instrument rule can be explicitly expressed as rational functions of the structural and implementable-path parameters, i.e. as fractions of polynomial functions of these parameters. In turn, these rational functions lend themselves easily to analytical manipulation. For instance, their derivatives

\footnotetext{
${ }^{3}$ In so doing, I extend and generalize McCallum's (1981) original result, which is in essence that, in this case, adding some private sector's actions or expectations to the policymaker's observation set may make some feasible paths implementable.

${ }^{4}$ For instance, this is typically the case of Taylor's (1993) popular interest-rate rule, and its extensions, in DSGE models of the monetary transmission mechanism. These rules require the policymaker to play simultaneously with the private sector or after it at each date. In Svensson's (1999) terminology, they are implicit.
} 
can be computed in order to determine how the coefficients of the policy-instrument rule respond to an arbitrarily small change in the value of the structural or implementable-path parameters.

This method of designing policy-instrument rules can be readily applied to any model encompassed by my general framework, any stabilization-policy instrument, any policymaker's observation set of a kind that I consider, and any path that is implementable for these model, policy instrument, and observation set. Of course, unless the specific model, observation set, and implementable path considered are particularly simple, these policy-instrument rules will typically be more complex than the policy-instrument rules commonly considered in the literature, like Taylor's (1993) interest-rate rule, in the sense of involving a larger number of variables. This greater complexity does not matter at all in the rational-expectations paradigm. However, it might raise concerns about the relevance of this paradigm in that context. Can these rules be viewed as more than interesting theoretical benchmarks to which to compare simple rules in rational-expectations models? I believe that they can. Actual decision procedures used by real-world policymakers amount to complex rules involving many inputs, yet this complexity does not seem to raise concerns of this kind. Svensson $(2003,2011)$ argues that, even within the realm of rational-expectations models, the central bank's complex reaction function need not be communicated to the private sector. Similarly, it can be argued that all that the private sector needs to know, for such a rule to effectively implement a given path as the unique local equilibrium, is the path itself (which the policymaker can communicate through the publication of conditional economic forecasts) and the existence of such a rule, but not the rule itself. One contribution of this paper is precisely to identify conditions under which there exists such a rule, and conditions under which there does not.

I apply these general results to some specific models, policy instruments, and feasible paths, namely to optimal monetary policy in Svensson and Woodford's (2005) model, monetary policy based on constantinterest-rate forecasts in Leitemo's (2003) model, debt-stabilizing tax policy in Schmitt-Grohé and Uribe's (1997) model, and optimal tax policy in one of the models considered by Correia, Farhi, Nicolini, and Teles (2013). Some of these applications shed new light on earlier results obtained in the literature. For instance, Schmitt-Grohé and Uribe (1997) consider, in the standard neoclassical growth model, a labor-income tax-rate rule that balances the government's budget or stabilizes the expected future stock of public debt, find that this rule leads to local equilibrium indeterminacy for many admissible and empirically relevant values of the structural parameters, and conclude by warning against the use of labor-income taxes to balance the budget or stabilize the expected future stock of public debt. I show that, in the same model, for empirically plausible observation sets of the tax authority, all feasible paths along which the government's budget is balanced or the expected future stock of public debt is stabilized are implementable for all admissible values of the structural parameters. This result implies that Schmitt-Grohé and Uribe's (1997) point is not against debt-stabilizing labor-income-tax policy per se, but only against one specific way of implementing this policy. Similarly, I show that, in Leitemo's (2003) model, for empirically plausible observation sets of the central bank, the constant-interest-rate forecast targeting path is implementable for all admissible values of the structural parameters (except possibly a set of values of measure zero). This result implies that, contrary to what a cursory reading of Leitemo's (2003) findings may suggest, monetary policy based on constant-interest-rate forecasts does not inherently generate local equilibrium indeterminacy.

Throughout the paper, I make three assumptions that are standard either in the general literature on stabilization policy, or in the more specific literature on optimal stabilization policy. First, I assume the existence of a commitment technology that ensures the credibility of policy-instrument rules both in and 
out of equilibrium. Second, I implicitly assume that the policymaker knows the value of the model's structural parameters, i.e. I abstract from any consideration of model uncertainty. And third, I focus on local equilibrium determinacy, that is to say that I abstract from the possible existence of non-local equilibria. In the context of interest-rate rules, as argued by Cochrane (2011), there is usually no solid economic reason to assume away the existence of non-local equilibria. The most common policy proposal to eliminate these equilibria, made initially by Christiano and Rostagno (2001) and Benhabib, SchmittGrohé, and Uribe (2002), discussed by Woodford (2003a, Chapter 2), and used notably by Atkeson, Chari, and Kehoe (2010), consists in switching from an interest-rate rule ensuring local equilibrium determinacy to a money-growth rule (possibly accompanied by a non-Ricardian fiscal policy) when the economy goes outside a specified neighborhood of the steady state considered. The interest-rate rules that I design (when the policy instrument is the interest rate) fit naturally into this proposal, insofar as they are followed by the central bank inside the specified neighborhood. In particular, they are thus consistent with Atkeson, Chari, and Kehoe's (2010) "sophisticated" approach to monetary policy.

This paper is not the first one to design explicit policy-instrument rules implementing a given feasible path as the unique local equilibrium. Some existing papers - e.g., Evans and Honkapohja (2006), Svensson and Woodford (2005, Subsections 4.1 and 4.3) - algebraically design explicit policy-instrument rules that are consistent with a given feasible path, but find that these rules fail to implement that path as the unique local equilibrium for many admissible and empirically relevant values of the structural parameters. To my knowledge, only one of them - namely, Svensson and Woodford (2005, Subsections 3.6 and 4.2, and Section 5) - algebraically designs explicit policy-instrument rules that implement a given feasible path as the unique local equilibrium for all admissible values of the structural parameters. However, each of these papers considers a specific simple model (typically the basic New Keynesian model or one of its variants), a specific stabilization-policy instrument (typically the interest rate), a specific feasible path (typically the timeless-perspective Ramsey-optimal feasible path), and, in effect, a specific policymaker's observation set (typically such that the policymaker's observation constraint is slack in the optimization programme defining the timeless-perspective Ramsey-optimal feasible path). My contribution is therefore to extend and generalize their results in terms of model, policy instrument, observation set, and feasible path. Making this contribution requires to develop a new - arithmetic method of designing policy-instrument rules, as Svensson and Woodford's (2005) algebraic method can be applied only to simple models, observation sets, and implementable paths.

Nor is this paper the first one to design, in a general framework, policy specifications that are consistent with a given feasible path and with no other local path satisfying the structural equations. Indeed, Giannoni and Woodford (2010) design, in a broad class of models, "target criteria" that are consistent with the timeless-perspective Ramsey-optimal path and with no other local path satisfying the structural equations. Moreover, these criteria have two additional properties that may facilitate their communication to the public: they involve only the past, current, or expected future values of the variables featuring in the objective function considered (when this function is purely quadratic), and their coefficients are independent of certain structural parameters (in particular, the parameters governing the stochastic process of the exogenous disturbances). ${ }^{5}$ However, these criteria are typically not explicit policy-instrument rules, and sometimes not even policy-instrument rules. ${ }^{6}$ They are not meant to describe how timeless-

\footnotetext{
${ }^{5}$ These two properties have been emphasized, to various degrees, in Giannoni and Woodford (2002, 2003, 2005, 2010), Svensson (1997, 2003, 2011), Svensson and Woodford (2005), and Woodford (2003a, Chapters 4, 7, and 8, 2007). In these papers, such criteria may be called "target rules," "targeting rules," or "specific targeting rules."

${ }^{6}$ Similarly, the policy-instrument rules that I design in a more restrictive but still fairly general framework in Loisel (2009), which not only implement any given path as the unique local equilibrium, but also eliminate equilibrium trajectories
} 
perspective Ramsey-optimal stabilization policy could be implemented, but are instead proposed as, in Svensson and Woodford's (2005) terminology, a "higher-level policy specification." In this optimal-policy context, my contribution is therefore to determine whether and how these criteria can be operationally implemented, i.e. whether and how the policymaker can set the policy instrument conditionally on her observation set to implement, as the unique local equilibrium, the path that is Ramsey-optimal from the timeless perspective subject to her observation constraint - whether this observation constraint is slack, as implicitly assumed by Giannoni and Woodford (2010), or binding.

Finally, this paper is also related to Bassetto $(2002,2004,2005)$. Indeed, like the latter, it tackles implementation problems in macroeconomics by requiring that the policymaker's out-of-equilibrium behavior be feasible - i.e., in my case, consistent with her observation set. However, there are three important differences. First, this paper addresses a general local-equilibrium-determinacy issue, while Bassetto's papers address some specific global-equilibrium-determinacy issues. Second, the constraints faced out of equilibrium by the policymaker are only informational in this paper, while they are both informational and physical in Bassetto's papers. Third, in this paper, playing after the private sector at each date would give the policymaker the advantage of being able to react out of equilibrium to the private sector's current actions or expectations. In Bassetto's papers, it gives her the same advantage, but also a disadvantage, as the private sector's current actions may then reduce the set of her possible actions.

The rest of the paper is organized as follows. Section 1 justifies the assumption that the policymaker does not observe the private sector's date- $t$ actions and expectations when setting her date- $t$ policy instrument. Section 2 illustrates, in the basic New Keynesian model, some of the results obtained in the paper. Section 3 presents a broad class of locally linearizable dynamic stochastic discrete-time infinitehorizon rational-expectations models, which includes most existing DSGE models. Section 4 provides, in this class of models, for various alternative policymaker's observation sets, and for any feasible path, conditions for this path to be implementable, and shows how to design a corresponding policy-instrument rule arithmetically. Section 5 applies these general results to, and derive their implications for, the implementation of some specific stabilization policies in some specific models. I then conclude and provide a technical appendix.

\section{Portrait of the Policymaker as the First Mover}

Throughout the paper, I will assume that the policymaker plays before the private sector at each date. This section justifies that assumption.

One key difference between a policymaker and a private sector is that the latter is made of many more individuals than the former. For the sake of the argument, suppose that the private sector is made of a continuum of individuals, while the policymaker is a single individual. Suppose further that each individual takes decisions at a finite frequency, due to information-collecting, information-processing, and decision-making frictions, and that private agents' decisions are not synchronized with each other. Then, the private sector takes its decisions in continuous time, while the policymaker takes hers at discrete times. ${ }^{7}$

\footnotetext{
gradually leaving the neighborhood of the steady state considered, are typically implicit, not explicit.

${ }^{7}$ In crisis times, these frictions may weaken, and the frequency of policy decisions increase. For instance, central banks have swiftly taken decisions outside their regularly scheduled meetings at the height of the recent financial crisis. Even in such circumstances, however, financial-market participants - considered collectively - still take decisions more frequently than central banks. Moreover, I focus in this paper on policy-instrument rules that ensure local equilibrium determinacy,
} 
To translate this timing of events into discrete time, one needs to define (i) a date as a given period of time, (ii) the value of the policy instrument at a given date as its average value during the corresponding period, and (iii) the private sector's decisions at a given date as the aggregation of private agents' decisions over the corresponding period. There are four alternative ways to define a date, and therefore as many candidate discrete-time translations of this timing of events. First, one can define a date as the period of time between two consecutive policy decisions. In this case, the value of the policy instrument at a given date is its constant value during the corresponding period, and the policymaker plays before the private sector at each date. Second, one can define a date as a period of time that includes exactly one policy decision, taken neither at the beginning nor at the end of this period. In this case, however, the continuous-time within-period timing of events has no faithful discrete-time order-of-moves translation: at each date, the policymaker plays neither entirely before nor entirely after the private sector, nor do they truly play simultaneously with each other. Third, one can define a date as a period of time that includes several policy decisions. At the limit, when the period includes an infinity of policy decisions, the policymaker and the private sector play simultaneously with each other at each date. However, this case is ill-suited to the study of the implementation of stabilization policy, which is the issue that I address in this paper. Indeed, what matters from my perspective is how the policymaker should set her policy instrument at each policy decision, not how she should set the average value of her policy instrument over a period that includes several policy decisions. Fourth, one can define a date as a period of time that includes sometimes one policy decision, sometimes none. However, by restricting the set of feasible paths in non-trivial and unusual ways, this case would both complicate the analysis and hinder the comparison with existing stabilization-policy models (in which the policy instrument can typically change at each date).

To sum up, the discrete-time order of moves that is best suited to the study of the implementation of stabilization policy, while allowing the policymaker to play at each date, is the one in which the policymaker plays before the private sector at each date. Note that this order of moves is not necessarily the one most commonly considered in the literature. For instance, as I argue in Subsection 2.2, most analyses of monetary policy based on a DSGE model implicitly assume that the central bank plays simultaneously with the private sector or after it at each date, because the interest-rate rules that they consider require the interest rate to react out of equilibrium to the private sector's current actions or expectations. In this context, such a timing choice can be justified by the fact that these models are aimed at being estimated on macroeconomic data, and that these data correspond to periods of time (typically quarters) that include at least one policy decision taken neither at the beginning nor at the end of the period. Therefore, there is no faithful discrete-time order-of-moves translation of the continuoustime within-period timing of events, as discussed above, and one may as well assume that the central bank plays simultaneously with the private sector or after it at each date. However, these papers do not address - nor are they aimed at addressing - the issue of monetary-policy implementation. By contrast, most analyses of monetary policy that address this issue or a closely related issue, from Poole (1970) and Sargent and Wallace (1975) to King and Wolman (2004), Svensson and Woodford (2005), and Atkeson, Chari, and Kehoe (2010), explicitly assume, as I do, that the central bank plays before the private sector at each date. ${ }^{8}$

\footnotetext{
and hence are meant to apply only in "normal times."

${ }^{8}$ One notable exception is provided by Eggertsson and Swanson (2008), who consider the same model as King and Wolman (2004), but assume instead that the central bank and the private sector play simultaneously with each other. In order to enable the date- $t$ interest rate to react to date- $t$ aggregate variables, they introduce a Walrasian auctioneer to whom the central bank submits an interest-rate schedule that is a function of these variables.
} 


\section{A New Keynesian Illustration}

This section illustrates, in the well known basic New Keynesian model, some of the general results obtained in the rest of the paper. I consider, in this model, several alternative observation sets for the central bank. For each observation set, I do three things. First, I determine the path that is Ramsey-optimal from Woodford's (1999a) timeless perspective subject to the central bank's observation constraint, i.e., in the terminology introduced at the beginning of the paper, the timeless-perspective Ramsey-optimal feasible path. Second, I provide conditions for this feasible path to be implementable. Third, when it is implementable, I design some corresponding interest-rate rules. The method used to design interest-rate rules in this section is a brute-force guess-and-verify method - arguably the one that comes most naturally to mind, but one that can be applied only to simple models, observation sets, and implementable paths, and therefore not the one that will be used to design policy-instrument rules in the rest of the paper. One of the goals of the section is precisely to illustrate this limitation of that guess-and-verify method.

I refer the reader to Woodford (2003a, Chapters 2, 4, and 6) and Galí (2008, Chapter 3) for a detailed presentation of the basic New Keynesian model. I restrict the analysis to the neighborhood of the zero-inflation steady state, log-linearize the equilibrium conditions in this neighborhood, and express all endogenous and exogenous variables as log-deviations from their values at that steady state. The model's agents are, in alphabetical order, a central bank $(\mathcal{C B})$, Nature $(\mathcal{N})$, and a private sector $(\mathcal{P S})$. Time is discrete, indexed by $t \in \mathbb{Z}$. At each date $t, \mathcal{C B}$ sets the interest rate $i_{t} ; \mathcal{N}$ draws the realizations of two i.i.d. exogenous shocks $\nu_{t}$ and $u_{t}$ such that $\mathbb{E}\left\{\nu_{t} u_{t-k}\right\}=0$ for any $k \in \mathbb{Z}$, where $\mathbb{E}\{$. $\}$ denotes the unconditional-mean operator; and $\mathcal{P S}$ sets the inflation rate $\pi_{t}$ and the output level $y_{t}$, which I will refer to as $\mathcal{P S}$ 's actions, and forms its expectations of its own future actions and of $\mathcal{C B}$ 's future interest rate. ${ }^{9}$ The only assumption that I make at this stage about the chronological order of these moves is that, at each date $t \in \mathbb{Z}, \mathcal{P S}$ plays after $\mathcal{C B}$ and $\mathcal{N}$ : after $\mathcal{C B}$, for the reasons I have put forward in Section 1; and after $\mathcal{N}$, in order to allow exogenous shocks to affect $\mathcal{P} \mathcal{S}$ 's actions and expectations contemporaneously.

\subsection{Structural Equations}

The behavior of $\mathcal{P S}$ is described by the following two structural equations:

$$
\begin{aligned}
y_{t} & =\mathbb{E}_{t}\left\{y_{t+1}\right\}-\sigma\left(i_{t}-\mathbb{E}_{t}\left\{\pi_{t+1}\right\}\right)+\nu_{t}, \\
\pi_{t} & =\beta \mathbb{E}_{t}\left\{\pi_{t+1}\right\}+\kappa\left(y_{t}-\phi \nu_{t}\right)+u_{t},
\end{aligned}
$$

where $\beta, \kappa, \phi, \sigma$ are such that $0<\beta<1, \kappa>0,0 \leq \phi<1, \sigma>0$, and $\mathbb{E}_{t}\{$.$\} denotes the rational-$ expectations operator conditionally on the observation set of $\mathcal{P S}$ when it sets $\pi_{t}$ and $y_{t}$ (with the convention $\mathbb{E}_{t}\left\{\pi_{t}\right\}=\pi_{t}$ and $\left.\mathbb{E}_{t}\left\{y_{t}\right\}=y_{t}\right){ }^{10} \quad$ I assume that this observation set, which I note $O_{t}^{\mathcal{P} \mathcal{S}}$, is made of all previous moves of all players: $O_{t}^{\mathcal{P} S}=\left\{\pi^{t-1}, y^{t-1}, i^{t}, \mathbb{E}^{t-1}\{\pi\}, \mathbb{E}^{t-1}\{y\}, \mathbb{E}^{t-1}\{i\}, \nu^{t}, u^{t}\right\}$, where, throughout the paper, for any variable $z$ and any date $t, z^{t}$ denotes the history of variable $z$ until

\footnotetext{
${ }^{9} \mathcal{N}$ also draws the realization of some sunspot shocks (as opposed to the fundamental shocks $\nu_{t}$ and $u_{t}$ ) on which $\mathcal{P} \mathcal{S}$ 's actions could depend in equilibrium if the interest-rate rule did not ensure local equilibrium determinacy. However, the goal of this section is precisely to design interest-rate rules that ensure local equilibrium determinacy (among other properties), so that these sunspot shocks will play no role in equilibrium. Therefore, for expositional clarity, I omit mention of these shocks in the main text, like the bulk of the related literature.

${ }^{10}$ These two structural equations are usually expressed in terms of the welfare-relevant output gap $x_{t} \equiv y_{t}-\phi \nu_{t}$, instead of the output level $y_{t}$. I do not write them in this familiar form because (i) it is more convenient, in the context of this paper, to express them in terms of some $\mathcal{P} \mathcal{S}$ 's actions that are directly observed by $\mathcal{C B}$, and (ii) I will naturally assume that $\mathcal{C B}$ directly observes the output level, not the welfare-relevant output gap.
} 
date $t$ included, i.e. $\left(z_{t-j}\right)_{j \in \mathbb{N}}$, while $\mathbb{E}^{t-1}\{z\}$ denotes the history of the private sector's expectations

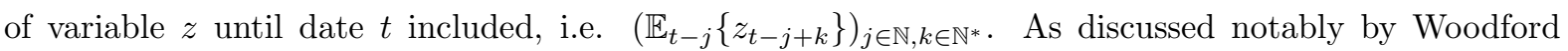
(2003a, Chapters 2, 4, and 6), each of the two exogenous shocks $\nu_{t}$ and $u_{t}$ can be interpreted in at least two different ways: $\nu_{t}$ may represent a fiscal-policy shock (exogenous variations in government purchases) or a preference shock (exogenous shifts in the consumption-utility function or in both the consumption-utility and the labor-disutility functions), while $u_{t}$ may represent another fiscal-policy shock (exogenous variations in distorting taxes on output, employment, or wage income) or a market-power shock (exogenous variations in the elasticity of substitution between differentiated goods). I will use these interpretations alternatively in Subsections 2.4 to 2.7 to justify my assumptions about $\mathcal{C B}$ 's observation set.

\section{$2.2 \quad$ Interest-Rate Rule}

Let $O_{t}^{\mathcal{C B}}$ denote the observation set of $\mathcal{C B}$ when it sets $i_{t}$. Since $\mathcal{C B}$ is assumed to play before $\mathcal{P S}$ at each date $t \in \mathbb{Z}$, all the sets $O_{t}^{\mathcal{C B}}$ that I will consider are included in the superset $\bar{O}_{t}^{\mathcal{C B}}$ made of $\mathcal{P} \mathcal{S}$ 's past actions and expectations, $\mathcal{C B}$ 's past interest rates, and $\mathcal{N}$ 's current and past exogenous shocks: $O_{t}^{\mathcal{C B}} \subset \bar{O}_{t}^{\mathcal{C B}} \equiv O_{t}^{\mathcal{P} \mathcal{S}} \backslash\left\{i_{t}\right\}=\left\{\pi^{t-1}, y^{t-1}, i^{t-1}, \mathbb{E}^{t-1}\{\pi\}, \mathbb{E}^{t-1}\{y\}, \mathbb{E}^{t-1}\{i\}, \nu^{t}, u^{t}\right\}$. The behavior of $\mathcal{C B}$ is described by an interest-rate rule. In the following, I will focus on interest-rate rules that express $i_{t}$ as a time-invariant function of a finite number of elements of $\bar{O}_{t}^{\mathcal{C B}}$ :

$$
\begin{aligned}
i_{t}= & \sum_{j=1}^{n^{e}} \sum_{k=1}^{n^{e}}\left(e_{j, k}^{\pi} \mathbb{E}_{t-j}\left\{\pi_{t-j+k}\right\}+e_{j, k}^{y} \mathbb{E}_{t-j}\left\{y_{t-j+k}\right\}+e_{j, k}^{i} \mathbb{E}_{t-j}\left\{i_{t-j+k}\right\}\right) \\
& +\sum_{j=1}^{n^{f}}\left(f_{j}^{\pi} \pi_{t-j}+f_{j}^{y} y_{t-j}\right)+\sum_{j=1}^{n^{g}} g_{j} i_{t-j}+\sum_{k=0}^{n^{h}}\left(h_{k}^{\nu} \nu_{t-k}+h_{k}^{u} u_{t-k}\right),
\end{aligned}
$$

where $\left(n^{e}, n^{f}, n^{g}\right) \in \mathbb{N}^{* 3}, n^{h} \in \mathbb{N}$, and all $e_{j, k}^{\pi}, e_{j, k}^{y}, e_{j, k}^{i}, f_{j}^{\pi}, f_{j}^{y}, g_{j}, h_{k}^{\nu}, h_{k}^{u}$ are real numbers.

The key feature of interest-rate rules of type (3) is that they involve no $\mathcal{P} \mathcal{S}$ 's current actions or expectations, consistently with my assumption that $\mathcal{C B}$ plays before $\mathcal{P} \mathcal{S}$ at each date. In the terminology first used by Svensson (1999), they are explicit. By contrast, most of the interest-rate rules considered in the DSGE literature are extensions of Taylor's (1993) rule that not only involve some $\mathcal{P} \mathcal{S}$ 's current actions or expectations, but also require the interest rate to react out of equilibrium to these current actions or expectations. ${ }^{11}$ Therefore, they require $\mathcal{C B}$ to observe the value taken by these current actions or expectations when setting the interest rate, and hence to play simultaneously with $\mathcal{P} \mathcal{S}$ or after it at each date. In Svensson's (1999) terminology, they are implicit. ${ }^{12}$

\subsection{Timeless-Perspective Ramsey-Optimal Feasible Path}

In this section, for the sake of illustration, I focus on a specific feasible path, namely, the timelessperspective Ramsey-optimal feasible path. This path can be defined as the limit of the date- $t_{0}$ Ramseyoptimal feasible path as $t_{0} \rightarrow-\infty$. The date- $t_{0}$ Ramsey-optimal feasible path is, in turn, defined as the

\footnotetext{
${ }^{11}$ Following the related literature, I implicitly define (i) the set of local paths as the set of paths that constantly remain in the neighborhood of the steady state considered, (ii) the set of equilibrium paths as the set of local paths that satisfy the structural equations and the policy-instrument rule at all dates, and (iii) the set of out-of-equilibrium paths as the set of local paths that satisfy the structural equations at all dates, but not the policy-instrument rule at all dates.

${ }^{12}$ The explicit vs. implicit terminology is also used by Svensson (2003) and Svensson and Woodford (2005), among others. Explicit interest-rate rules can also be encountered in the literature under the alternative names of "(explicit or implied) reaction functions" (Svensson, 1999, 2003; Svensson and Woodford, 2005; Woodford, 2003a, Chapter 7, 2007), "fundamentals-based reaction functions" (Evans and Honkapohja, 2006), "policy functions" (Svensson, 2011), and "backward-looking rules."
} 
state-contingent path for the endogenous variables that maximizes households' welfare at date $t_{0}$ subject to the structural equations and $\mathcal{C B}$ 's observation constraint. I assume for simplicity that the steady state considered is efficient (due to an employment or production subsidy offsetting the monopolisticcompetition distortion), so that the second-order approximation of the date- $t$ welfare loss function in the neighborhood of this steady state is of the form $L_{t}=\mathbb{E}_{t}\left\{\sum_{k=0}^{+\infty} \beta^{k}\left[\left(\pi_{t+k}\right)^{2}+\lambda\left(y_{t+k}-\phi \nu_{t+k}\right)^{2}\right]\right\}$, where $\lambda>0$.

\subsection{When $\mathcal{C B}$ Observes Past Actions and Current and Past Shocks}

I first consider the case in which $\mathcal{C B}$ observes all past actions, all current and past shocks, and no past expectations, i.e. $O_{t}^{\mathcal{C B}}=\left\{\pi^{t-1}, y^{t-1}, i^{t-1}, \nu^{t}, u^{t}\right\}$. The observation of all current and past shocks by $\mathcal{C B}$ can be justified by the interpretation of these shocks as fiscal-policy shocks (as discussed in Subsection 2.1) and the assumption that the government publicly announces its fiscal-policy decisions $\nu_{t}$ and $u_{t}$ at the beginning of period $t$, just before $\mathcal{C B}$ sets the interest rate. In this case, the timeless-perspective Ramsey-optimal feasible path is characterized by the following proposition:

Proposition 1 (timeless-perspective Ramsey-optimal feasible path, in the basic New Keynesian model, when $\mathcal{C B}$ observes past actions and current and past shocks): in the basic New Keynesian model, when $O_{t}^{\mathcal{C B}}=\left\{\pi^{t-1}, y^{t-1}, i^{t-1}, \nu^{t}, u^{t}\right\}$, the timeless-perspective Ramsey-optimal feasible path can be written in the following VARMA form:

$$
\begin{gathered}
{\left[\begin{array}{l}
\pi_{t} \\
y_{t} \\
i_{t}
\end{array}\right]=\left[\begin{array}{ccc}
0 & \frac{\lambda(1-\mu)}{\kappa} & 0 \\
0 & \mu & 0 \\
0 & \frac{-(\kappa-\lambda \sigma) \mu(1-\mu)}{\kappa \sigma} & 0
\end{array}\right]\left[\begin{array}{l}
\pi_{t-1} \\
y_{t-1} \\
i_{t-1}
\end{array}\right]+\left[\begin{array}{cc}
0 & \mu \\
\phi & \frac{-\kappa \mu}{\lambda} \\
\frac{1-\phi}{\sigma} & \frac{(\kappa-\lambda \sigma \mu(1-\mu)}{\lambda \sigma}
\end{array}\right]\left[\begin{array}{l}
\nu_{t} \\
u_{t}
\end{array}\right]} \\
+\left[\begin{array}{cc}
\frac{-\lambda \phi(1-\mu)}{\kappa} & 0 \\
\frac{-\phi \mu}{(\kappa-\lambda \sigma) \phi \mu(1-\mu)} & 0 \\
\kappa \sigma & 0
\end{array}\right]\left[\begin{array}{l}
\nu_{t-1} \\
u_{t-1}
\end{array}\right],
\end{gathered}
$$

where $\left.\mu \equiv \frac{\lambda+\beta \lambda+\kappa^{2}-\sqrt{\left(\lambda+\beta \lambda+\kappa^{2}\right)^{2}-4 \beta \lambda^{2}}}{2 \beta \lambda} \in\right] 0 ; 1[$.

Proof: see, e.g., Evans and Honkapohja (2006).

As is well known since Clarida, Galí, and Gertler (1999) and Woodford (1999a), the timeless-perspective Ramsey-optimal feasible path (4) is inertial in the presence of cost-push shocks, in the sense that it makes $\pi_{t}, y_{t}$, and $i_{t}$ depend on $u^{t-1}$. This inertia enables $\mathcal{C B}$ to make $\mathcal{P} \mathcal{S}$ 's current expectation of the future inflation rate $\mathbb{E}_{t}\left\{\pi_{t+1}\right\}$ depend negatively on the current cost-push shock $u_{t}$, and thus to relax the constraint imposed by the Phillips curve (2), in order to bring both the current inflation rate $\pi_{t}$ and the current welfare-relevant output gap $y_{t}-\phi \nu_{t}$ closer to zero. This inertia result has been viewed as a possible explanation for the observed smoothness of the interest-rate paths chosen by central banks over time. I will come back to this issue in the next subsection.

It is not straightforward to find an interest-rate rule of type (3) that involves no expectations and implements the timeless-perspective Ramsey-optimal feasible path (4) as the unique local equilibrium. One natural candidate is

$$
i_{t}=\frac{1-\phi}{\sigma} \nu_{t}+\sum_{k=0}^{+\infty} \frac{(\kappa-\lambda \sigma) \mu(1-\mu)}{\lambda \sigma} \mu^{k} u_{t-k},
$$


whose right-hand side is the value, as a function of only exogenous shocks, taken by the interest rate on the path (4) at date $t$. By construction, this rule is an interest-rate rule that involves no expectations and is consistent with the path (4). However, it is not of type (3), since it involves an infinite sum of current and past exogenous shocks. Moreover, as shown for instance by Woodford (2003a, Chapter 4), for any admissible values of the structural parameters $\beta, \kappa, \lambda, \phi$, and $\sigma$, this rule fails to ensure local equilibrium determinacy, i.e. fails to implement the path (4) as the unique local equilibrium. Another natural candidate is

$$
i_{t}=\frac{-(\kappa-\lambda \sigma) \mu(1-\mu)}{\kappa \sigma} y_{t-1}+\frac{1-\phi}{\sigma} \nu_{t}+\frac{(\kappa-\lambda \sigma) \mu(1-\mu)}{\lambda \sigma} u_{t}+\frac{(\kappa-\lambda \sigma) \phi \mu(1-\mu)}{\kappa \sigma} \nu_{t-1},
$$

which corresponds to the last line of the VARMA representation (4) of the timeless-perspective Ramseyoptimal feasible path. By construction, this rule is an interest-rate rule of type (3) that involves no expectations and is consistent with the path (4). However, as shown numerically by Evans and Honkapohja (2006) and discussed by Woodford (2003a, Chapter 7, 2007), for many admissible and empirically relevant values of the structural parameters $\beta, \kappa, \lambda, \phi$, and $\sigma$, this rule fails to ensure local equilibrium determinacy, i.e. fails to implement the path (4) as the unique local equilibrium. To my knowledge, there is no other attempt in the literature to find an interest-rate rule of type (3) that involves no expectations and implements the path (4) as the unique local equilibrium in the basic New Keynesian model. The following proposition aims at filling this gap in the literature by providing a non-empty parametric family of such rules:

Proposition 2 (implementation of the timeless-perspective Ramsey-optimal feasible path, in the basic New Keynesian model, when $\mathcal{C B}$ observes past actions and current and past shocks): any interest-rate rule of type $i_{t}=a \pi_{t-1}+b y_{t-1}+c \nu_{t}+d u_{t}+e \nu_{t-1}+f u_{t-1}$, where

$$
\begin{gathered}
a<\min \left\{-\frac{\kappa^{2} \sigma \mu+(1+\beta)\left[2 \kappa \mu+(\kappa-\lambda \sigma) \mu^{2}(1-\mu)\right]}{\beta \lambda \sigma\left(1-\mu^{2}\right)}, \frac{\kappa^{2} \sigma \mu+(1-\beta)(\kappa-\lambda \sigma) \mu^{2}(1-\mu)}{\beta \lambda \sigma(1-\mu)^{2}}\right\}, \\
b=\frac{-\lambda(1-\mu)}{\kappa \mu} a-\frac{(\kappa-\lambda \sigma) \mu(1-\mu)}{\kappa \sigma}, c=\frac{1-\phi}{\sigma}, d=\frac{(\kappa-\lambda \sigma) \mu(1-\mu)}{\lambda \sigma}, e=-\phi b, \text { and } f=-a,
\end{gathered}
$$

is such that the path (4) is the unique stationary solution of the system made of the IS equation (1), the Phillips curve (2), and this rule.

Proof: see Appendix A.1. This proposition is obtained as the outcome of a guess-and-verify procedure: guess that there exist some interest-rate rules of type $i_{t}=a \pi_{t-1}+b y_{t-1}+c \nu_{t}+d u_{t}+e \nu_{t-1}+f u_{t-1}$ with $(a, b, c, d, e, f) \in \mathbb{R}^{6}$ that implement the path (4) as the unique local equilibrium, and verify that this is indeed the case. Appendix A.1 simply shows that the condition on $(a, b, c, d, e, f)$ stated in Proposition 2 is sufficient for (i) the system made of the IS equation, the Phillips curve, and such an interest-rate rule to have a unique stationary solution $\left(\pi_{t}, y_{t}, i_{t}\right)_{t \in \mathbb{Z}}$, and (ii) the path (4) to be one stationary solution of this system.

In the terminology that I have introduced at the beginning of this paper, Proposition 2 implies that when $\mathcal{C B}$ observes all past actions, all current and past shocks, and no past expectations, the timelessperspective Ramsey-optimal feasible path is implementable for all admissible values of the structural parameters $\beta, \kappa, \lambda, \phi$, and $\sigma$. To understand how this proposition works, it is useful to decompose it into two results. The first result is that, for any admissible values of the structural parameters, there exists an interest-rate rule of type (3) involving no expectations and ensuring local equilibrium determinacy. In the context of the basic New Keynesian model, this result is not new: it has been obtained, 
for instance, by Bullard and Mitra (2002) and Carlstrom and Fuerst (2002). The second - stronger result is that, for any admissible values of the structural parameters, there exists an interest-rate rule of type (3) involving no expectations and implementing the timeless-perspective Ramsey-optimal feasible path as the unique local equilibrium. It might be thought at first sight that this second result (concerning the implementation of the timeless-perspective Ramsey-optimal feasible path) is a straightforward consequence of the first one (concerning local equilibrium determinacy), on the ground that adding an exogenous term to an interest-rate rule does not affect its ability to ensure local equilibrium determinacy and can make the interest rate take the same in-equilibrium value as on the path considered. Such a reasoning, however, would be wrong for two reasons.

First, the exogenous term that would need to be added to an arbitrary interest-rate rule of type (3) involving no expectations and ensuring local equilibrium determinacy, for the interest rate to take the same in-equilibrium value as on the path (4), would typically be written as an infinite sum of current and past exogenous shocks, so that the resulting rule would typically not be of type (3), as this type necessarily involves a finite (possibly degenerate) sum of these shocks. I overcome this difficulty by designing an interest-rate rule that not only ensures local equilibrium determinacy, but also makes the system made of the structural equations and itself admit the unique eigenvalue of the path (4) as one of its eigenvalues. ${ }^{13}$ In other words, I choose coefficients $a$ and $b$ not only such that the number of unstable eigenvalues of this system is 2 , but also such that the value of its stable eigenvalue is $\mu .^{14}$

Second, the unique local equilibrium implemented by such an interest-rate rule would not necessarily be the path (4). Indeed, even though the interest rate would, by construction, take the same in-equilibrium value under such an interest-rate rule as on the path (4), the inflation rate and the output level might not, as follows from Galî's (2011) analysis. ${ }^{15}$ I overcome this difficulty by designing an interest-rate rule that is satisfied on the path (4). Therefore, the path (4) is one stationary solution of the system made of the structural equations and this rule. Since this rule ensures local equilibrium determinacy, that system has a unique stationary solution, which must then coincide with the path (4).

Note finally that none of the interest-rate rules designed in Proposition 2 satisfies the long-run Taylor principle considered by Woodford (2003a, Chapter 4). Indeed, this principle states that if the inflation rate were permanently higher by one percentage point, then the system made of the Phillips curve (2) and the interest-rate rule should make the interest rate permanently higher by more than one percentage point, i.e. $a+\frac{1-\beta}{\kappa} b>1$. Now, as clear from Appendix A.1, all the interest-rate rules designed in Proposition 2 are, on the contrary, such that $a+\frac{1-\beta}{\kappa} b<1$. In fact, as clear from Condition (A.3) in Appendix A.1, for interest-rate rules of type $i_{t}=a \pi_{t-1}+b y_{t-1}+e_{t}$, where $e_{t}$ is an exogenous term, the long-run Taylor principle is not a necessary condition for local equilibrium determinacy in the basic New Keynesian model, even when both $a$ and $b$ are restricted to be positive. By contrast, for interest-rate rules of type $i_{t}=a \pi_{t}+b y_{t}+e_{t}$, where $e_{t}$ is again an exogenous term, this principle is a necessary

\footnotetext{
${ }^{13}$ Throughout the paper, by "eigenvalues" of a dynamic rational-expectations system, I mean the eigenvalues of the matrix that characterizes the corresponding deterministic system written in Blanchard and Kahn's (1980) form; and by "eigenvalues" of a path written in a VARMA form, I mean the eigenvalues of the matrix that characterizes the VAR part of this VARMA representation.

${ }^{14}$ Throughout the paper, I use the terms "stable" and "unstable" in the same sense as in the related literature, to mean "of modulus strictly lower than one" and "of modulus higher than or equal to one" respectively.

${ }^{15}$ Galí (2011) designs algebraically, in the same basic New Keynesian model (though without $\nu$ shocks), for any admissible value of the structural parameters and any exogenously specified interest-rate path, several interest-rate rules ensuring local equilibrium determinacy and such that the interest rate follows this path in each of the corresponding unique local equilibria. He then illustrates numerically, for specific values of the structural parameters and a specific (constant) exogenous interestrate path, that these equilibria differ from each other in terms of the path followed by the inflation rate and the output level.
} 
condition for local equilibrium determinacy in the same model when both $a$ and $b$ are restricted to be positive, as shown for instance by Woodford (2003a, Chapter 4).

\subsection{When $\mathcal{C B}$ Observes Past Actions and Past Shocks}

I now turn to the case in which $\mathcal{C B}$ observes all past actions, all past shocks, no current shocks, and no past expectations, i.e. $O_{t}^{\mathcal{C B}}=\left\{\pi^{t-1}, y^{t-1}, i^{t-1}, \nu^{t-1}, u^{t-1}\right\}$. The observation of all past shocks and no current shocks by $\mathcal{C B}$ can be justified by, again, the interpretation of these shocks as fiscal-policy shocks and, this time, the assumption that the government publicly announces its fiscal-policy decisions $\nu_{t}$ and $u_{t}$ at the beginning of period $t$, just after $\mathcal{C B}$ sets the interest rate. In this case, the timeless-perspective Ramsey-optimal feasible path is characterized by the following proposition:

Proposition 3 (timeless-perspective Ramsey-optimal feasible path, in the basic New Keynesian model, when $\mathcal{C B}$ observes past actions and past shocks): in the basic New Keynesian model, when $O_{t}^{\mathcal{C B}}=\left\{\pi^{t-1}, y^{t-1}, i^{t-1}, \nu^{t-1}, u^{t-1}\right\}$, the timeless-perspective Ramsey-optimal feasible path can be written in the following VARMA form:

$$
\left[\begin{array}{l}
\pi_{t} \\
y_{t} \\
i_{t}
\end{array}\right]=\left[\begin{array}{ccc}
0 & \frac{\lambda(1-\mu)}{\kappa} & 0 \\
0 & \mu & 0 \\
0 & \frac{-(\kappa-\lambda \sigma) \mu(1-\mu)}{\kappa \sigma} & 0
\end{array}\right]\left[\begin{array}{c}
\pi_{t-1} \\
y_{t-1} \\
i_{t-1}
\end{array}\right]+\boldsymbol{\Omega}_{0}\left[\begin{array}{c}
\nu_{t} \\
u_{t}
\end{array}\right]+\boldsymbol{\Omega}_{1}\left[\begin{array}{c}
\nu_{t-1} \\
u_{t-1}
\end{array}\right]+\boldsymbol{\Omega}_{2}\left[\begin{array}{c}
\nu_{t-2} \\
u_{t-2}
\end{array}\right],
$$

where $\boldsymbol{\Omega}_{0}, \boldsymbol{\Omega}_{1}$, and $\boldsymbol{\Omega}_{2}$ are $3 \times 2$ matrices whose elements are generically non-zero real numbers, except the two elements in the last line of $\boldsymbol{\Omega}_{0}$, which are equal to zero. ${ }^{16}$

Proof: see Appendix A.2.

Interestingly, unlike (4), the timeless-perspective Ramsey-optimal feasible path (5) is inertial even in response to $\nu$ shocks, in the sense that it makes $\pi_{t}, y_{t}$, and $i_{t}$ depend on $\nu^{t-1}$. To understand this new optimal-inertia result, consider for simplicity the case in which there are no cost-push shocks, i.e. $u_{t}=0$ for all $t \in \mathbb{Z}$. Since $i_{t}$ cannot react to $\nu_{t}$ (as $\nu_{t} \notin O_{t}^{\mathcal{C B}}$ ), the IS equation (1) prevents $\mathcal{C B}$ from achieving $\left(y_{t}, \mathbb{E}_{t}\left\{y_{t+1}\right\}, \mathbb{E}_{t}\left\{\pi_{t+1}\right\}\right)=\left(\phi \nu_{t}, 0,0\right)$. In such a context, this inertia enables $\mathcal{C B}$ to make $\mathbb{E}_{t}\left\{y_{t+1}\right\}+\sigma \mathbb{E}_{t}\left\{\pi_{t+1}\right\}$ depend negatively on $\nu_{t}$, and thus to relax the constraint imposed by the IS equation (1), in order to bring $y_{t}$ closer to $\phi \nu_{t}$ - and therefore, via the Phillips curve (2), to bring $\pi_{t}$ closer to zero.

Thus, Proposition 3 provides a new explanation for the observed smoothness of interest-rate paths chosen by central banks over time. Unlike the existing explanations of this stylized fact that also rest on a timeless-perspective Ramsey-optimal monetary policy, this new explanation does not rest on the existence of cost-push shocks (as in the previous subsection), nor on the existence of non-negligible transaction frictions that would make the second-order approximation of the instantaneous welfare loss function include a term in $i_{t}^{2}$ (as in Woodford, 1999b, 2000, 2003a, Chapter 7, 2003b). ${ }^{17}$ Instead, it rests here on the existence of an $\nu$ shock and the assumption that $\mathcal{C B}$ observes the past values but not the current value of this shock. Moreover, this new optimal-inertia result can be easily shown to obtain

\footnotetext{
${ }^{16}$ Throughout the paper, I use letters in bold to denote vectors and matrices that have (at least potentially) more than one element.

${ }^{17}$ Svensson $(2003$, p. 462) questions the quantitative relevance of these transaction frictions and the implied optimal inertia.
} 
for other shocks that also affect the natural rate of interest, like productivity shocks, provided that $\mathcal{C B}$ observes the past values - or infers them from its observation set - but not the current value of these shocks. ${ }^{18}$

Naturally, since the path (5) is absent from the literature, there has not been any attempt to find an interest-rate rule of type (3) that (i) involves no past expectations and no current shocks, and (ii) implements this path as the unique local equilibrium in the basic New Keynesian model. The following proposition aims at filling this gap in the literature by providing a non-empty parametric family of such rules:

Proposition 4 (implementation of the timeless-perspective Ramsey-optimal feasible path, in the basic New Keynesian model, when $\mathcal{C B}$ observes past actions and past shocks): any interest-rate rule of type $i_{t}=a \pi_{t-1}+b y_{t-1}+c \nu_{t-1}+d u_{t-1}+e \nu_{t-2}+f u_{t-2}$, where

$$
\begin{aligned}
& a<\min \left\{-\frac{\kappa^{2} \sigma \mu+(1+\beta)\left[2 \kappa \mu+(\kappa-\lambda \sigma) \mu^{2}(1-\mu)\right]}{\beta \lambda \sigma\left(1-\mu^{2}\right)}, \frac{\kappa^{2} \sigma \mu+(1-\beta)(\kappa-\lambda \sigma) \mu^{2}(1-\mu)}{\beta \lambda \sigma(1-\mu)^{2}}\right\}, \\
& b=\frac{-\lambda(1-\mu)}{\kappa \mu} a-\frac{(\kappa-\lambda \sigma) \mu(1-\mu)}{\kappa \sigma}, \text { and }\left[\begin{array}{l}
c \\
d \\
e \\
f
\end{array}\right]=\left[\begin{array}{l}
\boldsymbol{\Omega}_{0}^{\prime} \\
\boldsymbol{\Omega}_{1}^{\prime}
\end{array}\right]\left[\begin{array}{c}
-a \\
\frac{\lambda(1-\mu)}{\kappa \mu} a \\
0
\end{array}\right]+\left[\begin{array}{l}
\boldsymbol{\Omega}_{1}^{\prime} \\
\boldsymbol{\Omega}_{2}^{\prime}
\end{array}\right]\left[\begin{array}{l}
0 \\
0 \\
1
\end{array}\right],
\end{aligned}
$$

is such that the path (5) is the unique stationary solution of the system made of the IS equation (1), the Phillips curve (2), and this rule.

Proof: see Appendix A.3.

This proposition implies that when $\mathcal{C B}$ observes all past actions, all past shocks, no current shocks, and no past expectations, the timeless-perspective Ramsey-optimal feasible path is implementable for all admissible values of the structural parameters. It is obtained with the same guess-and-verify method as Proposition 2, and it can be explained and interpreted in the same way as Proposition 2. Note that the coefficients $a$ and $b$ satisfy the same constraints in both propositions. Indeed, as explained in the previous subsection, these coefficients are chosen in order to (i) ensure local equilibrium determinacy, and (ii) make the system made of the IS equation, the Phillips curve, and the interest-rate rule admit the eigenvalues of the path (5) as its own eigenvalues. Now, (i) the conditions for local equilibrium determinacy are the same in both cases, since the two parametric families of interest-rate rules defined in Propositions 2 and 4 have the same deterministic structure (of type $i_{t}=a \pi_{t-1}+b y_{t-1}$ ), and (ii) the two paths (4) and (5) have the same eigenvalues (in fact, the same unique eigenvalue $\mu$ ), since they have the same autoregressive structure.

\footnotetext{
${ }^{18}$ In the case of productivity shocks, the assumption that $\mathcal{C B}$ does not observe the period- $t$ shock at the beginning of period $t$ seems reasonable. Moreover, $\mathcal{C B}$ can infer the past productivity shocks from its observation of past output and employment levels. As an illustration, remove the i.i.d. fiscal-policy or preference shocks $\left(\nu_{t}\right)_{t \in \mathbb{Z}}$ from the model, and replace them with i.i.d. productivity shocks $\left(a_{t}\right)_{t \in \mathbb{Z}}$. Accordingly, introduce the production function $y_{t}=(1-\alpha) n_{t}+a_{t}$, remove $\nu_{t}$ from the IS equation (1), replace $\phi \nu_{t}$ by $\psi a_{t}$ in the Phillips curve (2), and replace $\phi \nu_{t+k}$ by $\psi a_{t+k}$ for $k \in \mathbb{N}$ in the welfare loss function, where $0<\alpha<1, \psi>0$, and $n_{t}$ denotes the employment level at date $t$. Then, the values taken by $\pi_{t}$ and $i_{t}$ on the corresponding timeless-perspective Ramsey-optimal feasible path can be straightforwardly obtained by replacing $\nu_{t-k}$ by $\frac{-\psi}{1-\phi} a_{t-k}$ for $k \in \mathbb{N}$ in (A.24) and (A.25) in Appendix A.2, while the values taken by $y_{t}$ and $n_{t}$ on this path can be residually obtained with the Phillips curve (2) and the production function. Clearly, this path is inertial, in the sense that it makes the $\pi_{t}, y_{t}, n_{t}$, and $i_{t}$ depend on $a^{t-1}$.
} 


\subsection{When $\mathcal{C B}$ Observes Past Actions and Past Expectations}

I then consider the case in which $\mathcal{C B}$ observes all past actions, all past expectations, and no current or past shocks, i.e. $O_{t}^{\mathcal{C B}}=\left\{\pi^{t-1}, y^{t-1}, i^{t-1}, \mathbb{E}^{t-1}\{\pi\}, \mathbb{E}^{t-1}\{y\}, \mathbb{E}^{t-1}\{i\}\right\}$. The non-observation of any current or past shock by $\mathcal{C B}$ can be justified by the interpretation of these shocks as preference and marketpower shocks (as discussed in Subsection 2.1). The observation of $\mathcal{P} \mathcal{S}$ 's expectations by $\mathcal{C B}$ deserves a short explanation. Strictly speaking, expectations are thoughts and, unless $\mathcal{C B}$ manages to monitor and correctly interpret private agents' brain activity, it cannot directly observe their expectations. In reality, central banks do not observe private agents' expectations, but may infer them from financial-market prices and survey responses. The proper way to proceed would be to explicitly introduce these financialmarket prices and survey responses as additional $\mathcal{P} \mathcal{S}$ 's actions, which $\mathcal{C B}$ would observe and from which it would infer the value taken, whether in or out of equilibrium, by $\mathcal{P} \mathcal{S}$ 's expectations. ${ }^{19}$ For simplicity and without any loss in generality, I proceed instead as if $\mathcal{C B}$ directly observed $\mathcal{P} \mathcal{S}$ 's expectations. I then get the following proposition:

Proposition 5 (timeless-perspective Ramsey-optimal feasible path and its implementation, in the basic New Keynesian model, when $\mathcal{C B}$ observes past actions and past expectations): in the basic New Keynesian model, when $O_{t}^{\mathcal{C B}}=\left\{\pi^{t-1}, y^{t-1}, i^{t-1}, \mathbb{E}^{t-1}\{\pi\}, \mathbb{E}^{t-1}\{y\}, \mathbb{E}^{t-1}\{i\}\right\}$, the timeless-perspective Ramsey-optimal feasible path is the path (5), and any interest-rate rule of type $i_{t}=\sum_{j=1}^{2}\left(\alpha_{j}^{\pi} \pi_{t-j}+\alpha_{j}^{y} y_{t-j}+\alpha_{j}^{i} i_{t-j}+\alpha_{j}^{e \pi} \mathbb{E}_{t-j}\left\{\pi_{t-j+1}\right\}+\alpha_{j}^{e y} \mathbb{E}_{t-j}\left\{y_{t-j+1}\right\}\right)$ with

$$
\left[\begin{array}{c}
\alpha_{1}^{\pi} \\
\alpha_{1}^{y} \\
\alpha_{1}^{i} \\
\alpha_{1}^{e \pi} \\
\alpha_{1}^{e y}
\end{array}\right]=\left[\begin{array}{l}
a \\
b \\
0 \\
0 \\
0
\end{array}\right]+\boldsymbol{\Omega}\left[\begin{array}{l}
c \\
d
\end{array}\right] \text { and }\left[\begin{array}{c}
\alpha_{2}^{\pi} \\
\alpha_{2}^{y} \\
\alpha_{2}^{i} \\
\alpha_{2}^{e \pi} \\
\alpha_{2}^{e y}
\end{array}\right]=\boldsymbol{\Omega}\left[\begin{array}{l}
e \\
f
\end{array}\right] \text {, where }(i) \quad \boldsymbol{\Omega} \equiv\left[\begin{array}{cc}
0 & 1 \\
1 & -\kappa(1-\phi) \\
\sigma & \kappa \phi \sigma \\
-\sigma & -(\beta+\kappa \phi \sigma) \\
-1 & -\kappa \phi
\end{array}\right]
$$

and (ii) $a, b, c, d, e$, and $f$ meet the conditions stated in Proposition 4, is such that the path (5) is the unique stationary solution of the system made of the IS equation (1), the Phillips curve (2), and this rule.

Proof: for each $j \in\{1,2\}$, the IS equation (1) and the Phillips curve (2) taken at dates $t-j$ can be used to express $\nu_{t-j}$ and $u_{t-j}$ as functions of $\pi_{t-j}, y_{t-j}, i_{t-j}, \mathbb{E}_{t-j}\left\{\pi_{t-j+1}\right\}$, and $\mathbb{E}_{t-j}\left\{y_{t-j+1}\right\}$. The replacement of $\nu_{t-1}, u_{t-1}, \nu_{t-2}$, and $u_{t-2}$ by these expressions in the interest-rate rule $(R)$ stated in Proposition 4 leads to the interest-rate rule $\left(R^{\prime}\right)$ stated in Proposition 5. This operation is neutral from the local-equilibrium-determinacy point of view because the system made of $(1),(2)$, and $(R)$ taken at all dates $t \in \mathbb{Z}$ is equivalent to the system made of (1), (2), and ( $R^{\prime}$ ) taken at all dates $t \in \mathbb{Z}$. Proposition 5 follows.

Proposition 5 implies that when $\mathcal{C B}$ observes all past actions, all past expectations, and no current or past shocks, the timeless-perspective Ramsey-optimal feasible path is implementable for all admissible values of the structural parameters. This proposition also designs a parametric family of corresponding

\footnotetext{
${ }^{19}$ For instance, in the basic New Keynesian model at hand, suppose that, in addition to the market for nominal one-period bonds, whose price is $i_{t}$, there is a market for inflation-indexed one-period bonds, whose price is $r_{t}$. Then the vector of $\mathcal{P} \mathcal{S}$ 's date- $t$ actions becomes $\mathbf{Y}_{t}=\left[\begin{array}{ccc}\pi_{t} & y_{t} & r_{t}\end{array}\right]^{\prime}$, and the system of structural equations becomes the system made of the IS equation (1), the Phillips curve (2), and the Fisher equation $r_{t}=i_{t}-\mathbb{E}_{t}\left\{\pi_{t+1}\right\}$. For any $k \in \mathbb{N}^{*}$, if $\left\{r_{t-k}, i_{t-k}\right\} \subset O_{t}^{\mathcal{C B}}$, then, using the Fisher equation taken at date $t-k, \mathcal{C B}$ can infer at date $t$, from its observation of $r_{t-k}$ and $i_{t-k}$, the value taken, whether in or out of equilibrium, by the short-term expectation $\mathbb{E}_{t-k}\left\{\pi_{t-k+1}\right\}$.
} 
interest-rate rules, i.e. interest-rate rules that (i) involve only past actions and past expectations, and (ii) implement the path (5) as the unique local equilibrium. The design of such rules may not seem easy at first sight because introducing either actions or expectations into a given rule, in order to make it consistent with a given path, may not be neutral from the local-equilibrium-determinacy point of view. The trick is to (i) start from a rule that implements this path as the unique local equilibrium, but involves past actions and past shocks (namely the rule designed by Proposition 4), (ii) use the structural equations to express past shocks as functions of past actions and expectations, and (iii) replace the past shocks in this rule by those expressions.

\subsection{When $\mathcal{C B}$ Observes Past Actions}

I finally consider the case in which $\mathcal{C B}$ observes all past actions, no past expectations, and no current or past shocks, i.e. $O_{t}^{\mathcal{C B}}=\left\{\pi^{t-1}, y^{t-1}, i^{t-1}\right\}$. The non-observation of any current or past shock by $\mathcal{C B}$ can be justified by, again, the interpretation of these shocks as preference and market-power shocks, and its non-observation of any past expectation by the absence of adequate surveys or financial markets. In this case, $\mathcal{C B}$ can still react out of equilibrium to all past inflation rates, output levels, and interest rates. And it has one degree of freedom in its choice of an interest-rate rule consistent with the timeless-perspective Ramsey-optimal feasible path, given that it observes, for each past date, three endogenous variables whose values on this path depend on only two exogenous shocks. Therefore, it might be thought at first sight that, in this case as well, the timeless-perspective Ramsey-optimal feasible path is implementable for all admissible values of the structural parameters. The following proposition shows, however, that it may not:

Proposition 6 (timeless-perspective Ramsey-optimal feasible path and its implementation, in the basic New Keynesian model, when $\mathcal{C B}$ observes past actions): in the basic New Keynesian model, when $O_{t}^{\mathcal{C B}}=\left\{\pi^{t-1}, y^{t-1}, i^{t-1}\right\}$, the timeless-perspective Ramsey-optimal feasible path is the path (5), and there exists $\left(\alpha_{j}^{\pi}, \alpha_{j}^{y}, \alpha_{j}^{i}\right)_{1 \leq j \leq 2} \in \mathbb{R}^{6}$ such that the following two statements are equivalent to each other:

(a) there exists an interest-rate rule of type (3) such that (i) $\forall(j, k) \in\left\{1, \ldots, n^{e}\right\}^{2}, e_{j, k}^{\pi}=e_{j, k}^{y}=e_{j, k}^{i}=0$, (ii) $\forall k \in\left\{1, \ldots, n^{h}\right\}, h_{k}^{\nu}=h_{k}^{u}=0$, and (iii) this path is the unique stationary solution of the system made of the IS equation (1), the Phillips curve (2), and that rule,

(b) the system made of the IS equation (1), the Phillips curve (2), and the interest-rate rule $i_{t}=$ $\sum_{j=1}^{2}\left(\alpha_{j}^{\pi} \pi_{t-j}+\alpha_{j}^{y} y_{t-j}+\alpha_{j}^{i} i_{t-j}\right)$ has a unique stationary solution.

Proof: see Appendix A.4. In short, Appendix A.4 first shows that there exists $\left(\alpha_{j}^{\pi}, \alpha_{j}^{y}, \alpha_{j}^{i}\right)_{1 \leq j \leq 2} \in \mathbb{R}^{6}$ such that the set of interest-rate rules that (i) involve only past actions, and (ii) are consistent with the path (5), is the set $\left\{K(L)\left[i_{t}-\sum_{j=1}^{2}\left(\alpha_{j}^{\pi} \pi_{t-j}+\alpha_{j}^{y} y_{t-j}+\alpha_{j}^{i} i_{t-j}\right)\right]=0 \mid K(L) \in \mathbb{R}[L]\right\}$, where $L$ denotes the lag operator and $\mathbb{R}[L]$ the set of polynomials in $L$ with real-number coefficients. It then shows that the ability of an interest-rate rule of type $K(L)\left[i_{t}-\sum_{j=1}^{2}\left(\alpha_{j}^{\pi} \pi_{t-j}+\alpha_{j}^{y} y_{t-j}+\alpha_{j}^{i} i_{t-j}\right)\right]$ to ensure local equilibrium determinacy does not depend on $K(L)$, so that either all the rules of this set ensure local equilibrium determinacy, or none of them does.

For any calibration of the model's structural parameters, I can determine numerically, using the algebraic expression of $\left(\alpha_{j}^{\pi}, \alpha_{j}^{y}, \alpha_{j}^{i}\right)_{1 \leq j \leq 2}$ provided in Appendix A.4, whether the timeless-perspective Ramseyoptimal feasible path is implementable when $\mathcal{C B}$ observes only past actions. Let me consider, for instance, 
Table 1: Galí's (2008, Chapter 3) and Woodford's (2003a, Chapter 4) calibrations of the basic New Keynesian model ${ }^{21}$

\begin{tabular}{clcc}
\hline \hline \multicolumn{1}{c}{ Deep parameters } & & \\
\hline \hline Notation & Description & Value in G & Value in W \\
\hline$\alpha$ & Labor elasticity of output & 0.67 & 0.75 \\
$\beta$ & Discount factor & 0.99 & 0.99 \\
$\gamma$ & Intertemporal elasticity of substitution & 1.00 & - \\
$\varepsilon$ & Steady-state elasticity of substitution between diff. goods & 6.00 & 7.88 \\
$\chi$ & Inverse of Frisch labor supply elasticity & 1.00 & 0.11 \\
$s$ & Steady-state ratio of government purchases to output & 0.00 & - \\
$\theta$ & Probability of not being allowed to change one's price & 0.67 & 0.67 \\
\hline \hline \multicolumn{1}{c}{ Reduced-form parameters } & & \\
\hline \hline Notation & Formula & Value in G & Value in W \\
\hline$\sigma$ & $(1-s) \gamma$ & 1.000 & 6.250 \\
$\kappa$ & $\frac{(1-\theta)(1-\beta \theta)[\alpha+(1+\chi-\alpha) \sigma]}{\sigma \theta\left[\alpha+\left(1+\chi \mathbb{1}_{W}-\alpha\right) \varepsilon\right]}$ & 0.125 & 0.022 \\
$\lambda$ & $\frac{\kappa}{\varepsilon}$ & 0.021 & 0.003 \\
$\phi$ & $\alpha\left(1-\mathbb{1}_{c l}\right)$ & 0.335 or 0 & 0.250 or 0 \\
\hline \hline
\end{tabular}

Galî's (2008, Chapter 3) and Woodford's (2003a, Chapter 4) calibrations of the basic New Keynesian model, which are described in Table 1. Moreover, for each of these two calibrations, let me consider two alternative cases. In the first case, $\nu_{t}$ comes from shifts in both the consumption-utility and the labor-disutility functions $\left(\mathbb{1}_{c l}=1\right.$ in Table 1$)$, and $u_{t}$ from variations in the elasticity of substitution between differentiated goods. In the second case, $\nu_{t}$ comes from shifts in the sole consumption-utility function $\left(\mathbb{1}_{c l}=0\right.$ in Table 1$)$, and $u_{t}$ from both variations in the elasticity of substitution between differentiated goods and shifts in the labor-disutility function. ${ }^{20}$ I find that, in both cases and for both calibrations, the timeless-perspective Ramsey-optimal feasible path is implementable when $\mathcal{C B}$ observes only past actions. I have also considered a grid of theoretically admissible values for the deep structural parameters (i.e. a grid of values for $\alpha, \beta, \gamma, \varepsilon, \chi, s$, and $\theta$ such that $0<\alpha \leq 1,0<\beta<1, \gamma>0$, $\varepsilon>1, \chi>0,0<s<1$, and $0<\theta<1$ ), and have obtained non-implementability only for empirically implausible values of these parameters. Finally, I have considered model-inconsistent values for $\lambda$, as often done in the applied literature (in which $\mathcal{C B}^{\text {'s }}$ loss function is not required to coincide with the welfare loss function), and have then obtained non-implementability for empirically relevant values of the deep structural parameters (e.g. for some of Table 1's values when $\lambda=1$ ).

\footnotetext{
${ }^{20}$ For expositional clarity, I have so far omitted mention of $\mathcal{P S}$ 's other actions (i.e., at date $t$, the consumption level $c_{t}$, hours worked $n_{t}$, and the real wage $w_{t}$ ) and the intra-temporal structural equations (i.e. the goods-market-clearing condition, the production function, and the labor vs. leisure trade-off). Clearly, this omission does not matter for the results of Subsections 2.4 to 2.6. Neither does it matter for the results of this subsection, because, in each of the two cases that I consider, whether the timeless-perspective Ramsey-optimal feasible path is implementable or not does not depend on whether $O_{t}^{\mathcal{C B}}$ includes or does not include $c^{t-1}, n^{t-1}$, and $w^{t-1}$ in addition to $\pi^{t-1}, y^{t-1}$, and $i^{t-1}$. Indeed, in the first case, the intra-temporal structural equations imply that $c_{t}, n_{t}$, and $w_{t}$ are proportional to $y_{t}$. And in the second case, they imply that $c_{t}$ and $n_{t}$ are proportional to $y_{t}$, and that $w_{t}$ is the only action (of $\mathcal{P S}$ or $\mathcal{C B}$ ) whose value on the timeless-perspective Ramsey-optimal feasible path does not depend only on $\nu^{t}$ and $u^{t}$ (as it also depends on a non-degenerate weighted sum of the consumption-utility and labor-disutility shocks at date $t$ ).

${ }^{21}$ The period is a quarter. "G" and "W" refer respectively to Galí's (2008, Chapter 3) and Woodford's (2003a, Chapter 4) calibrations, and $\mathbb{1}_{W}$ takes the value 0 in $G$ and 1 in $W$. The reason why the formula for $\kappa$ differs between $G$ and $W$ is that Galí (2008, Chapter 3) considers a single kind of labor, while Woodford (2003a, Chapter 4) considers differentiated kinds of labor. Finally, $\mathbb{1}_{c l}$ takes the value 0 when the exogenous shock $\nu_{t}$ comes from variations in government purchases or shifts in the consumption-utility function, and 1 when it comes from shifts in both the consumption-utility and the labor-disutility functions.
} 


\subsection{Discussion}

In this section, I have investigated, in the basic New Keynesian model, for several alternative central bank's observation sets, under which conditions - if any - the timeless-perspective Ramsey-optimal feasible path is implementable. However, even sticking with this model (the basic New Keynesian model) and that kind of feasible path (timeless-perspective Ramsey-optimal feasible paths), I have left aside interesting cases in terms of $\mathcal{C B}$ 's observation set. For instance, $\mathcal{C B}$ may not observe recent inflation rates and output levels, due to macroeconomic-data publication lags. In this case, $\mathcal{C B}$ cannot react out of equilibrium to these recent actions, which puts it further behind the curve, so that the conditions for the timeless-perspective Ramsey-optimal feasible path to be implementable are unclear at first sight. Moreover, this model (the basic New Keynesian model) and that kind of feasible path (timelessperspective Ramsey-optimal feasible paths) are specific in many ways. The analysis conducted in this section provides little clue as to what the implementability conditions would look like, for similar alternative policymaker's observation sets, in the same model for another kind of feasible path, in another model for the same kind of feasible path, or in another model for another kind of feasible path.

The brute-force guess-and-verify method that I have used to establish Propositions 2 and 4 (as well as, indirectly, Proposition 5) cannot be used to address these questions. Indeed, this method requires to (i) consider a given parametric family of policy-instrument rules, (ii) derive inequality conditions for these rules to ensure local equilibrium determinacy, i.e. for the characteristic polynomial of the implied system (made of the structural equations and the rule considered) to have a given number of unstable roots, (iii) derive equality conditions for these rules to be consistent with the feasible path considered, and (iv) show that there exist some rules meeting these inequality and equality conditions for all admissible values of the structural and feasible-path parameters (or else consider a larger parametric family of rules and start again). Now, the computations become intractable in practice as soon as the degree of the system's characteristic polynomial reaches the value four or five. ${ }^{22}$ Of course, this method could still be used to design policy-instrument rules numerically, but no general result could then be established.

In the next two sections, I will be able to address these questions, and generalize the results so far obtained in a specific example, by using a method to design policy-instrument rules arithmetically, i.e. by deriving their coefficients from the structural and implementable-path parameters with a finite number of arithmetic operations (addition, subtraction, multiplication, and division).

\section{The General Model}

This section presents the general dynamic stochastic discrete-time infinite-horizon rational-expectations model that will be used in the next section. I assume that this model admits at least one steady state in the neighborhood of which its equilibrium conditions are log-linearizable. I restrict the analysis to the neighborhood of this steady state, log-linearize the equilibrium conditions in that neighborhood, and express all endogenous and exogenous variables as log-deviations from their values at that steady state.

The model's agents are Nature $(\mathcal{N})$, a policymaker $(\mathcal{P} \mathcal{M})$, and a private sector $(\mathcal{P} \mathcal{S})$. Time is discrete,

\footnotetext{
${ }^{22}$ In the context of the basic New Keynesian model, the degree of this polynomial is equal to (i) three for the interest-rate rules considered in Propositions 2 and 4, (ii) two or three for all the interest-rate rules considered by Woodford (2003a, Chapter 4), and (iii) five or more, in the presence of observation lags (even of minimal length, i.e. of length one), for interest-rate rules that are consistent both with these observation lags and with the corresponding timeless-perspective Ramsey-optimal feasible path.
} 
indexed by $t \in \mathbb{Z}$. At each date $t$, the agents - taken in alphabetical order - move as follows: $\mathcal{N}$ draws the realization of exogenous shocks, $\mathcal{P} \mathcal{M}$ sets the policy instrument, and $\mathcal{P} \mathcal{S}$ sets its actions and forms its expectations of its own future actions and $\mathcal{P} \mathcal{M}$ 's future policy instrument. ${ }^{23}$ The only assumption that I make at this stage about the chronological order of these moves is that, at each date $t \in \mathbb{Z}$, $\mathcal{P S}$ plays after $\mathcal{N}$ and $\mathcal{P} \mathcal{M}$ : after $\mathcal{N}$, in order to allow exogenous shocks to affect $\mathcal{P} \mathcal{S}$ 's actions and expectations contemporaneously; and after $\mathcal{P} \mathcal{M}$, for the reasons I have put forward in Section 1.

\subsection{Exogenous Disturbances}

The behavior of $\mathcal{N}$ consists in drawing, at each date $t \in \mathbb{Z}$, the realization of $\varepsilon_{t}$, which is a centered white-noise $M_{2}$-dimension vector of exogenous shocks such that $M_{2} \in \mathbb{N}^{*}$ and $\mathbb{E}\left\{\varepsilon_{t} \varepsilon_{t-k}^{\prime}\right\}=\mathbf{0}$ for any $k \in \mathbb{Z}^{*}$. I then introduce the $M_{1}$-dimension vector of exogenous disturbances $\boldsymbol{\xi}_{t}$, where $M_{1} \in \mathbb{N}^{*}$, which follows a centered stationary finite-order VARMA process driven by $\varepsilon_{t}$ :

$$
\mathbf{A}_{1}(L) \boldsymbol{\xi}_{t}=\mathbf{A}_{2}(L) \varepsilon_{t}
$$

$$
\text { with } \underset{\left(M_{1} \times M_{1}\right)}{\mathbf{\mathbf { A } _ { 1 }}}(L) \equiv \sum_{k=0}^{n^{a_{1}}} \mathbf{A}_{1, k} L^{k} \text { and } \underset{\left(M_{1} \times M_{2}\right)}{\mathbf{A}_{2}(L)} \equiv \sum_{k=0}^{n^{a_{2}}} \mathbf{A}_{2, k} L^{k}
$$

where $\left(n^{a_{1}}, n^{a_{2}}\right) \in \mathbb{N}^{2}$, all $\mathbf{A}_{1, k}$ and $\mathbf{A}_{2, k}$ have real numbers as elements, $\mathbf{A}_{1}(0)$ is invertible, and all the roots of $\left|X^{n^{a_{1}}} \mathbf{A}_{1}\left(X^{-1}\right)\right|$ are stable (i.e. of modulus strictly lower than one). ${ }^{24}$ For simplicity and without any loss in generality, I also assume that $\mathbf{A}_{1}(L)$ is diagonal.

\subsection{Structural Equations}

Let $i_{t}$ denote the policy instrument at date $t$, and $\mathbf{Y}_{t}^{a}$ the $N^{a}$-dimension vector of $\mathcal{P} \mathcal{S}$ 's actions at date $t$, where $N^{a} \in \mathbb{N}^{*}$. Let $\mathbb{E}_{t}\{$.$\} denotes the rational-expectations operator conditionally on the observation$ set of $\mathcal{P} \mathcal{S}$ when it sets $\mathbf{Y}_{t}^{a}$ (with the convention $\mathbb{E}_{t}\left\{\mathbf{Y}_{t}^{a}\right\}=\mathbf{Y}_{t}^{a}$ ). I assume that this observation set, which I note $O_{t}^{\mathcal{P S}}$, is made of all previous moves of all players: $O_{t}^{\mathcal{P} S}=\left\{\mathbf{Y}^{a, t-1}, i^{t}, \mathbb{E}^{t-1}\left\{\mathbf{Y}^{a}\right\}, \mathbb{E}^{t-1}\{i\}, \varepsilon^{t}\right\}$. Let $\mathbf{Y}_{t}^{e}$ an $N^{e}$-dimension vector of expectations formed by $\mathcal{P S}$ at date $t$, where $N^{e} \in \mathbb{N}$, i.e. a vector whose elements belong to the set made of the elements of $\mathbb{E}_{t}\left\{\mathbf{Y}_{t+k}^{a}\right\}$ and $\mathbb{E}_{t}\left\{i_{t+k}\right\}$ for $k \in \mathbb{N}^{*}$. Finally, let $\mathbf{Y}_{t} \equiv\left[\begin{array}{cc}\mathbf{Y}_{t}^{a \prime} & \mathbf{Y}_{t}^{e \prime}\end{array}\right]^{\prime}$. The behavior of $\mathcal{P S}$ is described the following $N \equiv N^{a}+N^{e}$ structural equations:

$$
\mathbb{E}_{t}\left\{\mathbf{B}(L) \mathbf{Y}_{t}+\mathbf{C}(L) i_{t}\right\}+\mathbf{D}(L) \boldsymbol{\xi}_{t}=\mathbf{0}
$$

with $\underset{(N \times N)}{\mathbf{B}(L)} \equiv \sum_{k=-m^{b}}^{n^{b}} \mathbf{B}_{k} L^{k}, \underset{(N \times 1)}{\mathbf{C}(L)} \equiv \sum_{k=-m^{c}}^{n^{c}} \mathbf{C}_{k} L^{k}$, and $\underset{\left(N \times M_{1}\right)}{\mathbf{D}(L)} \equiv \sum_{k=0}^{n^{d}} \mathbf{D}_{k} L^{k}$,

where $\left(m^{b}, m^{c}, n^{b}, n^{c}, n^{d}\right) \in \mathbb{N}^{5} ;$ all $\mathbf{B}_{k}, \mathbf{C}_{k}$, and $\mathbf{D}_{k}$ have real numbers as elements; $L$ denotes the lag operator; and $\mathbf{0}$, throughout the paper, denotes a vector or a matrix whose elements are all equal to zero (and whose dimension depends on the specific context in which it is used).

Note that the system of structural equations (7) explicitly features an arbitrary finite number of lags and expected leads of $\mathcal{P} \mathcal{S}$ 's actions, its expectations, and the policy instrument. As is well known, this system could be rewritten in an equivalent reduced form with no lag and only one expected lead of a vector of endogenous variables: $\widetilde{\mathbf{B}}_{-1} \mathbb{E}_{t}\left\{\widetilde{\mathbf{Y}}_{t+1}\right\}+\widetilde{\mathbf{B}}_{0} \widetilde{\mathbf{Y}}_{t}+\widetilde{\mathbf{C}}_{t}+\widetilde{\mathbf{D}} \widetilde{\boldsymbol{\xi}}_{t}=\mathbf{0}$, where $\widetilde{\mathbf{B}}_{-1}, \widetilde{\mathbf{B}}_{0}, \widetilde{\mathbf{C}}$, and $\widetilde{\mathbf{D}}$ are

\footnotetext{
${ }^{23}$ These actions should be interpreted in a broad sense: in most applications, they will typically include both quantities and prices.

${ }^{24}$ As in Section 2, for expositional clarity, I omit mention of sunspot shocks (as opposed to the fundamental shocks $\boldsymbol{\varepsilon}_{t}$ ) in the main text, like the bulk of the related literature.
} 
matrices that have real numbers as elements, and $\widetilde{\mathbf{Y}}_{t}, \widetilde{i}_{t}$, and $\widetilde{\boldsymbol{\xi}}_{t}$ are vectors whose elements belong to the set made of the elements of, respectively, $\mathbb{E}_{t}\left\{\mathbf{Y}_{t+k}\right\}, \mathbb{E}_{t}\left\{i_{t+k}\right\}$, and $\mathbb{E}_{t}\left\{\boldsymbol{\xi}_{t+k}\right\}$ for $k \in \mathbb{Z}$. As a matter of fact, it is typically in this reduced form that the existing literature (e.g., Svensson, 1999, 2011; Giannoni and Woodford, 2002; Woodford, 2003a, Chapter 8) writes a general system of structural equations. I depart from the existing literature in this respect because I want to distinguish between $\mathcal{P} \mathcal{S}$ 's actions and its expectations, between its past and its current actions or expectations, and between the past and the expected future policy instruments, since I will assume that $\mathcal{P M}$ may observe the ones but not the others when she sets the current policy instrument. This reduced form, which mixes up all these actions and expectations with each other within the same vectors $\widetilde{\mathbf{Y}}_{t}$ and $\widetilde{i}_{t}$, would therefore be ill-suited for my purpose.

For each $j \in\{1, \ldots, N\}$, let $\mathbf{e}_{j}$ denote the $N$-element vector whose $j^{\text {th }}$ element is equal to one and whose other elements are equal to zero. Let $J_{B}$ denote the set of $j \in\{1, \ldots, N\}$ such that $\mathbf{e}_{j}^{\prime} \mathbf{B}(L) \neq \mathbf{0}$, and $J_{C}$ the set of $j \in\{1, \ldots, N\}$ such that $\mathbf{e}_{j}^{\prime} \mathbf{C}(L) \neq 0$. Let $m_{j}^{b} \equiv-\min \left[k \in\left\{-m^{b}, \ldots, n^{b}\right\}, \mathbf{e}_{j}^{\prime} \mathbf{B}_{k} \neq \mathbf{0}\right]$ for each $j \in J_{B}$, and $m_{j}^{c} \equiv-\min \left[k \in\left\{-m^{c}, \ldots, n^{c}\right\}, \mathbf{e}_{j}^{\prime} \mathbf{C}_{k} \neq 0\right]$ for each $j \in J_{C}$. Lastly, when $J_{B}=\{1, \ldots, N\}$, let $\widehat{\mathbf{B}}(L) \equiv\left[\begin{array}{lll}L^{m_{1}^{b}} \mathbf{e}_{1} & \cdots & L^{m_{N}^{b}} \mathbf{e}_{N}\end{array}\right] \mathbf{B}(L)$. I make the following four assumptions on $\mathbf{B}(L)$ and $\mathbf{C}(L)$ :

Assumption 1: $J_{B}=\{1, \ldots, N\}$.

Assumption 2: $\forall j \in\{1, \ldots, N\}, m_{j}^{b} \geq 0$.

Assumption 3: $\widehat{\mathbf{B}}(0)$ is invertible.

Assumption 4: $J_{C} \neq \varnothing$.

These assumptions are not restrictive at all. Indeed, any relevant model should satisfy Assumptions 1 and 2 for the structural equations to represent $\mathcal{P} \mathcal{S}$ 's choice of $\mathbf{Y}_{t}$, and Assumption 4 for the policy instrument to have an effect on this choice. Then, given Assumptions 1, 2 and 4, Assumption 3 is made without any loss in generality, since any system of type (6) and (7) satisfying Assumptions 1, 2 and 4 but not Assumption 3 can straightforwardly be rewritten in an equivalent form of type (6) and (7) either satisfying Assumptions 1 to 4, or not satisfying Assumption 1.

\subsection{Comparison with Existing DSGE Models}

Many - if not most - existing DSGE models are such that their exogenous disturbances can be written in a locally linearized form of type (6), and their structural equations in a locally linearized form of type (7) that satisfies Assumptions 1 to 4. Consider, for instance, the basic New Keynesian model considered in the previous section, which is the backbone of most existing DSGE models. Its exogenous disturbances are trivially of type (6) with $M_{1}=M_{2}=2$, and its structural equations (1) and (2) are already written in a form of type (7) with $N^{a}=2$ and $N^{e}=0$ that satisfies Assumptions 1 to 4 for all admissible values of the structural parameters $\beta, \kappa, \lambda, \phi$, and $\sigma$. Now consider a typical medium-scale DSGE model, such as Smets and Wouters' (2007): its exogenous disturbances are of type (6) with $M_{1}=M_{2}=6$ (ignoring the monetary-policy disturbance), and its structural equations are easily shown to be already written in a form of type (7) with $N^{a}=13$ and $N^{e}=0$ that satisfies Assumptions 1 to 4 for all admissible values of its structural parameters, except a set of values of measure zero. ${ }^{25}$

\footnotetext{
${ }^{25}$ As can be easily checked, these structural equations, as they are written by Smets and Wouters (2007), satisfy Assumption 3 unless $\left(1-c_{1}\right)\left[z_{1} z_{y}-\alpha \phi_{p} z_{1}-(1-\alpha) \phi_{p}\right]+c_{2} c_{y}=0$, where $c_{1}, c_{2}, c_{y}, z_{1}, z_{y}, \alpha$, and $\phi_{p}$ are reduced-form parameters, and the set of structural-parameter values such that this equality is satisfied is of measure zero.
} 
In particular, my general framework allows for three interesting features. First, it allows for the presence of policy transmission lags of the kind considered by, e.g., Rotemberg and Woodford (1999), Woodford (2003a, Chapter 5), Christiano, Eichenbaum, and Evans (2005), and Svensson and Woodford (2005). In these models, $\mathcal{P S}$ decides unconditionally in advance on its actions, so that the structural equations make its current actions depend on its past expectations. My framework allows for the presence of such lags - whatever their length - because $\mathcal{P} \mathcal{S}$ 's past expectations may feature in the structural equations (7) when $N^{e} \geq 1$. In the literature, policy transmission lags are often considered in the case of monetary policy, given the delay with which an unexpected monetary-policy decision affects the economy.

Second, it allows for the presence of policy implementation lags of the kind considered by Schmitt-Grohé and Uribe (1997, Section III). These lags compel $\mathcal{P} \mathcal{M}$ to choose unconditionally in advance the value of her policy instrument. My framework captures the presence of such lags of an arbitrary length $l \in \mathbb{N}^{*}$ when all variables of type $\mathbb{E}_{t}\left\{i_{t+k}\right\}$ with $k \in \mathbb{Z}$ in the structural equations (7) are such that $k \leq-l$, i.e. when $\forall j \in\{1, \ldots, N\}, m_{j}^{c} \leq-l$. In the literature, policy implementation lags are most often considered in the case of fiscal policy, given the relatively long period of time that is typically observed in practice between the announcement and the implementation of fiscal-policy decisions. ${ }^{26}$

Third, it allows for the presence of news shocks of the kind considered by Christiano, Motto, and Rostagno (2013). To see this possibility, consider for simplicity the case in which $M_{1}=1 .{ }^{27}$ Assume that the unique element of $\boldsymbol{\xi}_{t}$, noted $\xi_{t}$, follows the stochastic process $\sum_{k=0}^{n^{a_{1}}} a_{1, k} \xi_{t-k}=\sum_{k=0}^{n^{a_{2}}} a_{2, k} \varphi_{k, t-k}$, where $n^{a_{1}} \in \mathbb{N}$, $n^{a_{2}} \in \mathbb{N}^{*}$, all $a_{1, k}$ and $a_{2, k}$ are real numbers, $a_{1,0} \neq 0$, all the roots of $\sum_{k=0}^{n^{a_{1}}} a_{1, k} X^{n^{a_{1}}-k}$ are stable, and, for each $k \in\left\{0, \ldots, n^{a_{2}}\right\}, \varphi_{k, t-k}$ is a shock whose realization is observed by $\mathcal{P S}$ at date $t-k$. For each $k \in\left\{1, \ldots, n^{a_{2}}\right\}, \varphi_{k, t}$ is a "news shock" in the sense that it gives to $\mathcal{P} \mathcal{S}$ at date $t$ some information about $\xi_{t+k}$ without affecting $\xi_{t+j}$ for $j \in\{0, \ldots, k-1\}$. Assume further that the vector of date- $t$ news shocks $\boldsymbol{\varphi}_{t} \equiv\left[\begin{array}{lll}\varphi_{1, t} & \cdots & \varphi_{n^{a_{2}, t}}\end{array}\right]$ is such that $\mathbb{E}\left\{\boldsymbol{\varphi}_{t} \boldsymbol{\varphi}_{t-k}^{\prime}\right\}=\mathbb{1}_{k=0} \mathbf{W}$ for any $k \in \mathbb{Z}$, where $\mathbf{W}$ is a symmetric positive semi-definite $\left(n^{a_{2}}+1\right) \times\left(n^{a_{2}}+1\right)$ matrix. Then, the stochastic process of $\xi_{t}$ can easily be rewritten in a form of type (6) with $\mathbf{A}_{1}(L) \equiv\left[\sum_{k=0}^{n^{a_{1}}} a_{1, k} L^{k}\right], \mathbf{A}_{2}(L) \equiv\left[\begin{array}{llll}a_{2,0} & a_{2,1} L & \cdots & a_{2, n^{a_{2}}} L^{n^{a_{2}}}\end{array}\right] \mathbf{W}^{\frac{1}{2}}$, and $\varepsilon_{t} \equiv \mathbf{W}^{-\frac{1}{2}} \varphi_{t}$.

\subsection{Policy-Instrument Rule}

Let $O_{t}^{\mathcal{P} \mathcal{M}}$ denote the observation set of $\mathcal{P} \mathcal{M}$ when she sets $i_{t}$. Since $\mathcal{P} \mathcal{M}$ is assumed to play before $\mathcal{P} \mathcal{S}$ at each date $t \in \mathbb{Z}$, all the sets $O_{t}^{\mathcal{P} \mathcal{M}}$ that I will consider are included in the set $\bar{O}_{t}^{\mathcal{P} \mathcal{M}}$ made of $\mathcal{P} \mathcal{S}$ 's past actions and expectations, $\mathcal{P} \mathcal{M}$ 's past policy instruments, and $\mathcal{N}$ 's current and past exogenous shocks: $O_{t}^{\mathcal{P} \mathcal{M}} \subset \bar{O}_{t}^{\mathcal{P} \mathcal{M}} \equiv O_{t}^{\mathcal{P} \mathcal{S}} \backslash\left\{i_{t}\right\}=\left\{\mathbf{Y}^{a, t-1}, i^{t-1}, \mathbb{E}^{t-1}\left\{\mathbf{Y}^{a}\right\}, \mathbb{E}^{t-1}\{i\}, \varepsilon^{t}\right\}$. The behavior of $\mathcal{P} \mathcal{M}$ is described by a policy-instrument rule. Throughout the rest of the paper, I will focus on policy-instrument rules that express $i_{t}$ as a time-invariant function of a finite number of elements of $\bar{O}_{t}^{\mathcal{P} \mathcal{M}}$, and I find it convenient to write them in a form that involves the vector $\mathbf{Y}_{t}$, rather than the vector $\mathbf{Y}_{t}^{a}$ :

$$
\mathbf{E}_{1}(L) \mathbb{E}_{t}\left\{\mathbf{E}_{2}\left(L^{-1}\right)\left[\begin{array}{ll}
\mathbf{Y}_{t}^{\prime} & i_{t}
\end{array}\right]^{\prime}\right\}+\mathbf{F}(L) \mathbf{Y}_{t}+G(L) i_{t}+\mathbf{H}(L) \varepsilon_{t}=0
$$

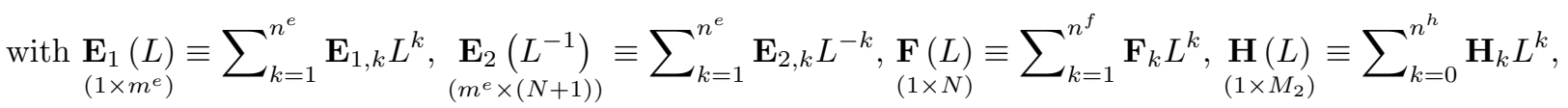

\footnotetext{
${ }^{26}$ For instance, defining conservatively the date of announcement of a bill as the date when the bill becomes law, Morten and Ravn (2010) find that around half of the "exogenous" U.S. tax bills since World War II were announced more than one quarter ahead of their implementation date, and that the median implementation lag (i.e. the median period of time between the announcement date and the implementation date) for these bills is six quarters.

${ }^{27}$ The reasoning can straightforwardly be generalized to cases in which $M_{1}>1$. Christiano, Motto, and Rostagno (2013) consider news shocks about future technology, monetary-policy, government-spending, equity, and risk disturbances.
} 
and $G(L) \equiv \sum_{k=0}^{n^{g}} g_{k} L^{k}$, where $\left(m^{e}, n^{e}, n^{f}\right) \in \mathbb{N}^{* 3},\left(n^{g}, n^{h}\right) \in \mathbb{N}^{2}$, all $g_{k}$ are real numbers, $g_{0} \neq 0$, and all $\mathbf{E}_{1, k}, \mathbf{E}_{2, k}, \mathbf{F}_{k}, \mathbf{H}_{k}$ have real numbers as elements. The key feature of policy-instrument rules of type (8) is that they involve no $\mathcal{P} \mathcal{S}$ 's current actions or expectations, i.e. they are such that $\mathbf{E}_{1}(0)=\mathbf{0}$ and $\mathbf{F}(0)=\mathbf{0}$, consistently with my assumption that $\mathcal{P} \mathcal{M}$ plays before $\mathcal{P S}$ at each date $t \in \mathbb{Z}$. In the terminology first used by Svensson (1999), they are explicit. As I have argued in Subsection 2.2 in the context of DSGE models of the monetary transmission mechanism, most of the policy-instrument rules considered in the literature, on the contrary, do involve some $\mathcal{P} \mathcal{S}$ 's current actions or expectations, and do require $\mathcal{P} \mathcal{M}$ to play after $\mathcal{P} \mathcal{S}$ at each date $t \in \mathbb{Z}$. In Svensson's (1999) terminology, they are implicit.

\subsection{Superset of Feasible Paths}

Since all the observation sets $O_{t}^{\mathcal{P M}}$ that I will consider are included in the set $\bar{O}_{t}^{\mathcal{P M}}$ defined in the previous subsection, all the feasible paths that I will consider belong to the superset of paths that are consistent with the structural equations and the observation set $\bar{O}_{t}^{\mathcal{P} \mathcal{M}}$. For simplicity, I focus on paths that can be written in a finite-order centered stationary VARMA process driven by the vector of exogenous shocks $\varepsilon_{t} \cdot{ }^{28}$ And, for convenience, I consider paths for $\left[\begin{array}{cc}\mathbf{Y}_{t}^{\prime} & i_{t}\end{array}\right]^{\prime}$, rather than $\left[\begin{array}{cc}\mathbf{Y}_{t}^{a \prime} & i_{t}\end{array}\right]^{\prime}$. Therefore, the superset of feasible paths is the set of paths that can be written in the form

$$
\begin{gathered}
\mathbf{S}(L)\left[\begin{array}{ll}
\mathbf{Y}_{t}^{\prime} & i_{t}
\end{array}\right]^{\prime}=\mathbf{T}(L) \varepsilon_{t} \\
\text { with } \underset{((N+1) \times(N+1))}{\mathbf{S}(L)} \equiv \sum_{k=0}^{n^{s}} \mathbf{S}_{k} L^{k} \text { and } \underset{\left((N+1) \times M_{2}\right)}{\mathbf{T}(L)} \equiv \sum_{k=0}^{n^{t}} \mathbf{T}_{k} L^{k},
\end{gathered}
$$

where $\left(n^{s}, n^{t}\right) \in \mathbb{N}^{2}$; all $\mathbf{S}_{k}$ and $\mathbf{T}_{k}$ have real numbers as elements; $\mathbf{S}_{0}$ is the $(N+1) \times(N+1)$ identity matrix $\mathbf{I}_{N+1}$; all the roots of $\left|X^{n^{s}} \mathbf{S}\left(X^{-1}\right)\right|$ are stable; and $\mathbf{S}(L)$ and $\mathbf{T}(L)$ are such that the path $\left(\mathbf{Y}_{t}, i_{t}\right)_{t \in \mathbb{Z}}$ defined by (9) satisfies the structural equations (7). ${ }^{29}$ For each $j \in\{1, \ldots, N+1\}$, let $\mathbf{v}_{j}$ denote the $(N+1)$-element vector whose $j^{\text {th }}$ element is equal to one and whose other elements are equal to zero. Without any loss in generality, I also impose $\mathbf{S}(L)$ to be such that if $N^{e} \geq 1$, then $\left[\begin{array}{llll}\mathbf{v}_{1} & \ldots & \mathbf{v}_{N^{a}}\end{array}\right]^{\prime} \mathbf{S}(L)\left[\begin{array}{lll}\mathbf{v}_{N^{a}+1} & \ldots & \mathbf{v}_{N}\end{array}\right]=\mathbf{0}$, so that the first $N^{a}$ lines of $(9)$ do not involve any $\mathcal{P} \mathcal{S}$ 's expectations.

\section{Design of Policy-Instrument Rules}

In this section, I provide, in the general model of the previous section, for various alternative $\mathcal{P} \mathcal{M}$ 's observation sets, and for any feasible path, conditions for this path to be implementable. In addition, when it is implementable, I show how to design some corresponding policy-instrument rules arithmetically.

\subsection{A Preliminary Result}

Before proceeding, let me establish a preliminary result that will prove useful to design policy-instrument rules in the rest of the paper. Let $\mathbb{R}[X]$ denote the set of polynomials in $X$ with real-number coefficients,

\footnotetext{
${ }^{28}$ This focus on finite-order VARMA processes is not restrictive in the sense that, as straightforwardly follows from Blanchard and Kahn's (1980) analysis, the unique stationary solution of a linear time-invariant finite-order rationalexpectations model with finite-order VARMA disturbances necessarily follows a finite-order VARMA process, provided that this model can be written in Blanchard and Kahn's (1980) form.

${ }^{29}$ In fact, Assumptions 1 to 4 do not guarantee that the system of structural equations (7) has at least one stationary solution $\left(\mathbf{Y}_{t}, i_{t}\right)_{t \in \mathbb{Z}}$. Naturally, the results that I will obtain in the next section make sense only if it does.
} 
and, for each $j \in\{1, \ldots, N+1\}$, let $\Delta_{j}(X) \in \mathbb{R}[X]$ denote the determinant of the $N \times N$ matrix obtained by removing its $j^{\text {th }}$ column from $N \times(N+1)$ matrix $X^{\max \left\{n^{b}, n^{c}\right\}}\left[\mathbf{B}\left(X^{-1}\right) \mid \mathbf{C}\left(X^{-1}\right)\right]$. I then get the following lemma:

Lemma 1: for any policy-instrument rule of type (8) such that

$$
\begin{gathered}
\mathbf{E}_{1}(L)=\mathbf{0}, \\
\text { if } \max _{j \in J_{C}}\left(m_{j}^{c}-m_{j}^{b}\right) \geq 1, \text { then } \forall k \in\left\{1, \ldots, \max _{j \in J_{C}}\left(m_{j}^{c}-m_{j}^{b}\right)\right\} \cap\left\{1, \ldots, n^{f}\right\}, \mathbf{F}_{k}=\mathbf{0},
\end{gathered}
$$

the system made of the structural equations (7) and this rule can be written in Blanchard and Kahn's (1980) form with $m \equiv \sum_{j=1}^{N} m_{j}^{b}$ non-predetermined variables, and its non-zero eigenvalues are (and have the same multiplicity as) the non-zero roots of polynomial

$$
X^{\max \left\{n^{f}, n^{g}\right\}}\left[\sum_{j=1}^{N}(-1)^{N+1-j} \mathbf{F}\left(X^{-1}\right) \mathbf{e}_{j} \Delta_{j}(X)+G\left(X^{-1}\right) \Delta_{N+1}(X)\right] .
$$

Proof: see Appendix B.1. $\mathbf{\square}$ In short, Appendix B.1 essentially uses (i) Assumption 3 to show that the system mentioned in Lemma 1 has exactly $m$ non-predetermined variables, and (ii) a standard result of time-series analysis (see, e.g., Hamilton, 1994, Chapter 10, Proposition 10.1) to show that the non-zero eigenvalues of this system are the non-zero roots of (12).

Most of the policy-instrument rules of type (8) satisfying (10) that I will design in the rest of the paper also satisfy (11), so that I will be conveniently able to use Lemma 1 to design them. ${ }^{30}$

\subsection{When $\mathcal{P} \mathcal{M}$ Observes Actions and Shocks, Possibly with Lags}

I first consider the case in which $\mathcal{P} \mathcal{M}$ observes all actions, all shocks, and no expectations. In addition, I allow for the possibility that $\mathcal{P} \mathcal{M}$ observes the actions of $\mathcal{P} \mathcal{S}$ and shocks with some lags. More specifically, I assume that there exists $\left(l_{Y}, l_{\varepsilon}\right) \in \mathbb{N}^{2}$ such that $l_{\varepsilon} \leq l_{Y}+1$ and $O_{t}^{\mathcal{P M}}=\left\{\mathbf{Y}^{a, t-1-l_{Y}}, i^{t-1}, \varepsilon^{t-l_{\varepsilon}}\right\} .^{31}$ When $l_{Y}=l_{\varepsilon}=0$, there are no observation lags (as, for instance, in Subsection 2.4). When $l_{Y} \geq 1$ or $l_{\varepsilon} \geq 1, \mathcal{P} \mathcal{M}$ does not observe recent actions or shocks (as, for instance, in Subsection 2.5, where $l_{Y}=0$ and $l_{\varepsilon}=1$ ). The existence of observation lags can be justified by that of macroeconomic-data publication lags. ${ }^{32}$ McCallum (1999), in particular, argues that policy-instrument rules for monetary policy need to take such lags into account to be "operational," and Orphanides (2001) points out that interest-rate rules commonly considered in the literature involve variables that are, in fact, not known with any accuracy until several quarters later. These publication lags may apply not only to $\mathcal{P} \mathcal{S}$ 's actions, but also to exogenous shocks, for instance to foreign macroeconomic data (considered as exogenous from the point of view of a small open economy).

\footnotetext{
${ }^{30}$ Most DSGE models without policy transmission lags are such that $\max \left\{m_{j}^{c}-m_{j}^{b} \mid j \in J_{C}\right\} \leq 0$, so that all policyinstrument rules of type (8) meet condition (11) in these models. However, DSGE models with policy transmission lags are often such that $\max \left\{m_{j}^{c}-m_{j}^{b} \mid j \in J_{C}\right\} \geq 1$. Such is the case, for instance, of Svensson and Woodford's (2005) model, as apparent from Subsection 5.1's analysis.

${ }^{31}$ The analysis can be straightforwardly extended to the case in which $\mathcal{P} \mathcal{M}$ observes only some actions of $\mathcal{P} \mathcal{S}$, instead of all of them, i.e. to the case in which there exist $\left(l_{Y}, l_{\varepsilon}\right) \in \mathbb{N}^{2}$ and a set $I$ such that $l_{\varepsilon} \leq l_{Y}+1, \varnothing \varsubsetneqq I \varsubsetneqq\left\{1, \ldots, N^{a}\right\}$, and $O_{t}^{\mathcal{P} \mathcal{M}}=\left\{\mathbf{e}_{i}^{\prime} \mathbf{Y}^{t-1-l_{Y}}, i^{t-1}, \varepsilon^{t-l_{\varepsilon}} \mid i \in I\right\}$. In practice, one example of $\mathcal{P} \mathcal{S}$ 's action that $\mathcal{P} \mathcal{M}$ may not observe, even with a $\mathrm{lag}$, is the capital utilization rate in the service sector.

${ }^{32}$ Some variables, most notably some financial-market variables, can clearly be observed without any lag by the policymaker. However, as argued by Leeper, Sims and Zha (1996, p. 40) and Rotemberg and Woodford (1999, pp. 93-94), the policymaker may still react with some lags to these variables because of data-processing and decision-making frictions.
} 
I assume, mostly for convenience, that observation lags on shocks are not too large relatively to observation lags on $\mathcal{P S}$ 's actions, i.e. $l_{\varepsilon} \leq l_{Y}+1 .{ }^{33}$ Of course, when $l_{\varepsilon} \leq l_{Y}$, if $\mathcal{P} \mathcal{M}$ manages to implement a given feasible path as the unique local equilibrium, then she will be able to recover, at each date $t \in \mathbb{Z}$, from her observation of the exogenous shocks $\varepsilon^{t-l_{\varepsilon}}$, the value taken in equilibrium by the actions of $\mathcal{P} \mathcal{S}$ that she does not observe, namely $\mathbf{Y}_{t-l_{\varepsilon}-k}$ for $k \in\left\{0, \ldots, l_{Y}-l_{\varepsilon}\right\}$. However, the relevant question is whether her inability to react out of equilibrium to these actions prevents her from implementing that path as the unique local equilibrium in the first place.

In this case, the rules of type (8) that are consistent with $O_{t}^{\mathcal{P} \mathcal{M}}$ are those that satisfy (10),

$$
\begin{aligned}
& \text { if } N^{e} \geq 1, \text { then } \mathbf{F}(L)\left[\begin{array}{lll}
\mathbf{e}_{N^{a}+1} & \ldots & \mathbf{e}_{N}
\end{array}\right]=\mathbf{0}, \\
& \text { if } l_{Y} \geq 1, \text { then } \forall k \in\left\{1, \ldots, l_{Y}\right\} \cap\left\{1, \ldots, n^{f}\right\}, \mathbf{F}_{k}\left[\begin{array}{lll}
\mathbf{e}_{1} & \ldots & \mathbf{e}_{N^{a}}
\end{array}\right]=\mathbf{0} \text {, } \\
& \text { and if } l_{\varepsilon} \geq 1 \text {, then } \forall k \in\left\{0, \ldots, l_{\varepsilon}-1\right\} \cap\left\{0, \ldots, n^{h}\right\}, \mathbf{H}_{k}=\mathbf{0} \text {, }
\end{aligned}
$$

while the feasible paths, i.e. the paths of type (9) that are consistent with $O_{t}^{\mathcal{P}}$, are those that satisfy

$$
\begin{aligned}
\text { if } N^{e} \geq 1, \text { then } & \mathbf{v}_{N+1}^{\prime} \mathbf{S}(L)\left[\begin{array}{lll}
\mathbf{v}_{N^{a}+1} & \ldots & \mathbf{v}_{N}
\end{array}\right]=\mathbf{0}, \\
\text { if } l_{Y} \geq 1, \text { then } & \forall k \in\left\{1, \ldots, l_{Y}\right\} \cap\left\{1, \ldots, n^{s}\right\}, \mathbf{v}_{N+1}^{\prime} \mathbf{S}_{k}\left[\begin{array}{lll}
\mathbf{v}_{1} & \ldots & \mathbf{v}_{N^{a}}
\end{array}\right]=\mathbf{0}, \\
\text { and if } l_{\varepsilon} \geq 1, & \text { then } \quad \forall k \in\left\{0, \ldots, l_{\varepsilon}-1\right\} \cap\left\{0, \ldots, n^{t}\right\}, \mathbf{v}_{N+1}^{\prime} \mathbf{T}_{k}=\mathbf{0} .
\end{aligned}
$$

Let $\mathcal{D}(X) \in \mathbb{R}[X]$ denote the greatest common divisor, defined up to a non-zero real-number multiplicative scalar, of all non-zero $\Delta_{j}(X)$ for $j \in\left\{1, \ldots, N^{a}\right\} \cup\{N+1\}$. I then obtain the following proposition, which generalizes Propositions 2 and 4 to any policy, any model, any observation-lag length, and any feasible path:

Proposition 7 (feasible-path implementation, in the general model, when $\mathcal{P} \mathcal{M}$ observes actions and shocks, possibly with lags): if $\mathcal{D}(X)$ has at most $m$ unstable roots (taking into account their multiplicity), then, for any $\left(l_{Y}, l_{\varepsilon}\right) \in \mathbb{N}^{2}$ such that $l_{\varepsilon} \leq l_{Y}+1$, for any path $\left(\mathbf{Y}_{t}, i_{t}\right)_{t \in \mathbb{Z}}$ of type (9) satisfying (16), (17), and (18), there exists an arithmetically designable policy-instrument rule of type (8) satisfying (10), (13), (14), and (15), such that this path $\left(\mathbf{Y}_{t}, i_{t}\right)_{t \in \mathbb{Z}}$ is the unique stationary solution of the system made of the structural equations (7) and that rule.

Proof: see Appendix B.2. In short, Appendix B.2 proceeds as follows. It first applies Bézout's identity to $\left(\Delta_{i}(X)\right)_{i \in\left\{1, \ldots, N^{a}\right\} \cup\{N+1\}}$, i.e. it shows the existence of $\left(\mathcal{U}_{i}(X)\right)_{i \in\left\{1, \ldots, N^{a}\right\} \cup\{N+1\}} \in \mathbb{R}[X]^{N^{a}+1}$ such that $\sum_{i \in\left\{1, \ldots, N^{a}\right\} \cup\{N+1\}} \mathcal{U}_{i}(X) \Delta_{i}(X)=\mathcal{D}(X) .{ }^{34}$ It then uses the Euclidian division to design, from $\left(\mathcal{U}_{i}(X)\right)_{i \in\left\{1, \ldots, N^{a}\right\} \cup\{N+1\}}$, some $\mathbf{F}(L)$ and $G(L)$ such that (i) $\mathbf{F}(L)$ satisfies (11), (13), and (14), (ii) (12) has exactly $m$ unstable roots (taking into account their multiplicity), and (iii) (12) admits the roots of $\left|X^{n^{s}} \mathbf{S}\left(X^{-1}\right)\right|$ as stable roots. At this stage, given Lemma 1, any policy-instrument rule of type (8) with $\mathbf{E}_{1}(L)=\mathbf{0}$ and these $\mathbf{F}(L)$ and $G(L)$ is such that (i) the system made of the structural equations (7) and

\footnotetext{
${ }^{33}$ Clearly, all the implementation lags described in Subsection 3.3 can be equivalently rewritten as observation lags. The reverse, however, is not true because no implementation lags can mimick the effects of observation lags of lengths $l_{Y}$ and $l_{\varepsilon}$ such that $l_{Y} \neq l_{\varepsilon}$.

${ }^{34}$ Bézout's identity is named after Étienne Bézout (1730-1783), who extended to polynomials a result first obtained for integers by Claude-Gaspard Bachet de Méziriac (1581-1638). It is sometimes unnamed and presented as a corollary of the Euclidean algorithm (see, e.g., Prasolov, 2004, Chapter 2, Theorem 2.1.1). I have searched for papers in economics that use Bézout's identity, and have found only d'Autume (1990) and Loisel (2009). D'Autume (1990) uses it to characterize the set of solutions of a linear scalar equation with rational expectations and ARMA disturbances. And in Loisel (2009), I use it to design policy-instrument rules that are typically not of type (8).
} 
this rule has at most one stationary solution, since it meets Blanchard and Kahn's (1980) order condition (but not necessarily their rank condition), and (ii) the eigenvalues of the feasible path (9) considered are also eigenvalues of this system. Finally, Appendix B.2 uses Cramer's rule to residually choose $\mathbf{H}(L)$ satisfying (15) and such the feasible path (9) considered is one, and hence the unique, stationary solution of this system. ${ }^{35}$

Proposition 7 provides, when $\mathcal{P} \mathcal{M}$ observes actions and shocks (possibly with lags), for each feasible path, a sufficient condition for this path to be implementable. In addition, it says that, when this condition is met, a corresponding policy-instrument rule can be designed arithmetically. To understand how this proposition works, it is useful to decompose it into three results of increasing strength. The first result is that, for any system of structural equations of type (7) satisfying Assumptions 1 to 4 , for any $\mathcal{P} \mathcal{M}$ 's observation set of a kind considered in this subsection, under a certain condition that does not involve the observation-lag length $l_{Y} \in \mathbb{N}$, there exists a policy-instrument rule consistent with this observation set and ensuring local equilibrium determinacy. ${ }^{36}$ This result is not obvious. Most of the policy-instrument rules considered in the literature, at least in the context of DSGE models of the monetary transmission mechanism, manage to ensure local equilibrium determinacy by requiring the policy instrument to react out of equilibrium to $\mathcal{P} \mathcal{S}$ 's current actions or expectations. Against this background, by preventing $\mathcal{P} \mathcal{M}$ from reacting out of equilibrium to these current actions or expectations, my timing assumption thus puts $\mathcal{P} \mathcal{M}$ behind the curve. ${ }^{37}$ Policy implementation or observation lags, when present, put her still further behind the curve by preventing her from reacting out of equilibrium to $\mathcal{P} \mathcal{S}$ 's recent actions or expectations. $^{38}$ The way $\mathcal{P} \mathcal{M}$ can still ensure local equilibrium determinacy in such a situation is via $\mathcal{P S}$ 's expectation that she will go on following her policy-instrument rule in the future. Indeed, in models that raise non-trivial local-equilibrium-indeterminacy issues, the structural equations make $\mathbf{Y}_{t}$ depend on both $\mathbb{E}_{t}\left\{i_{t+j}\right\}$ and $\mathbb{E}_{t}\left\{\mathbf{Y}_{t+k}\right\}$ for some $j \in \mathbb{Z}$ and $k \in \mathbb{N}^{*}$. By recurrence, they also make $\mathbf{Y}_{t}$ depend on $\mathbb{E}_{t}\left\{\mathbf{Y}_{t+n k}\right\}$ for some $n \in \mathbb{N}^{*}$ such that $n k \geq-j+l_{Y}+1$. Therefore, they make $\mathbf{Y}_{t}$ depend indirectly on $\mathbb{E}_{t}\left\{i_{t+n k+j}\right\}$. Now, by imposing an out-of-equilibrium reaction of $i_{t}$ to $\mathbf{Y}_{t-(n k+j)}$, a policy-instrument rule also imposes an out-of-equilibrium reaction of $\mathbb{E}_{t}\left\{i_{t+n k+j}\right\}$ to $\mathbf{Y}_{t}$. The latter reaction can be viewed as the feedback mechanism that ensures local equilibrium determinacy. ${ }^{39}$

The second - stronger - result is that, for any system of structural equations of type (7) satisfying Assumptions 1 to 4, for any $\mathcal{P} \mathcal{M}$ 's observation set of a kind considered in this subsection, under the same condition as the one previously mentioned, all feasible paths are implementable. As in Subsection 2.4 (in the specific context of the basic New Keynesian model, no observation lags, and the timeless-

\footnotetext{
${ }^{35}$ Cramer's rule is named after Gabriel Cramer (1704-1752), who generalized to an arbitrary finite number of unknowns a result first obtained for two or three unknowns by Colin Maclaurin (1698-1746). This rule expresses the unique solution of a system of linear equations with as many equations as unknowns as a function of the determinants of some matrices.

${ }^{36}$ The simplest way to illustrate this result is to consider the limit of Section 2's basic New Keynesian model as the degree of price stickiness tends towards zero and the elasticity of substitution between differentiated goods towards infinity (see, e.g., Galí, 2008, Chapter 2, for a detailed presentation of this "classical monetary model"). In the deterministic version of the resulting model, the only structural equation that involves $\mathcal{P} \mathcal{S}$ 's expectations is the Fisher equation $\mathbb{E}_{t}\left\{\pi_{t+1}\right\}-i_{t}=0$ and, for any $l_{Y} \in \mathbb{N}$, the rule $i_{t}=\alpha \pi_{t-l_{Y}-1}$ with $-1<\alpha<1$ clearly ensures local equilibrium determinacy.

${ }^{37}$ In Eggertsson and Swanson's (2008, p. 3) words: "if the government moves first, this curbs its ability to react to the behavior of the private sector in that period, limiting its ability to exclude self-fulfilling prophecies."

${ }^{38}$ If anything, the existing literature suggests that such lags may reduce the policymaker's ability to ensure local equilibrium determinacy. For instance, Benhabib (2004, Subsection 3.4) considers, in the context of a specific model, a parametric family of simple interest-rate rules that are consistent with observation lags of arbitrary length $l \in \mathbb{N}^{*}$. Holding constant the values of the model's parameters other than $l$, he finds numerically that local equilibrium indeterminacy arises for sufficiently large values of $l$.

${ }^{39}$ A similar idea is expressed in Rotemberg and Woodford (1999, p. 109) and Svensson and Woodford (2005, p. 75) in the context of models with monetary-policy transmission lags. In Rotemberg and Woodford's (1999, p. 109) words, for instance: "lags in the effects of a monetary policy change do not imply that an effective policy must be 'forward-looking.' The crucial insight is that there is no need for policy to be forward-looking as long as the private sector is." (The emphasis is theirs.)
} 
perspective Ramsey-optimal feasible path), it might be thought at first sight that this second result (concerning feasible-path implementation) is a straightforward consequence of the first one (concerning local equilibrium determinacy), on the ground that adding an exogenous term to a policy-instrument rule does not affect its ability to ensure local equilibrium determinacy and can make the policy instrument take the same in-equilibrium value as on the feasible path considered. Such a reasoning, however, would be wrong for essentially the same two reasons as in Subsection 2.4.

First, the exogenous term that would need to be added to an arbitrary policy-instrument rule of type (8) consistent with $O_{t}^{\mathcal{P M}}$ and ensuring local equilibrium determinacy, for the policy instrument to take the same in-equilibrium value as on the feasible path considered, would typically be written as an infinite sum of current and past exogenous shocks, so that the resulting rule would typically not be of type (8), as this type requires that $\mathbf{H}(L) \boldsymbol{\xi}_{t}$ be a finite (possibly degenerate) sum of these shocks. I overcome this difficulty by designing a policy-instrument rule that not only ensures local equilibrium determinacy, but also makes the system made of the structural equations and itself admit the eigenvalues of the feasible path considered as (at least some of) its own eigenvalues. I do this by using Bézout's identity to choose not only the number of unstable eigenvalues of this system, but also the values of (at least some of) its stable eigenvalues.

Second, the unique local equilibrium implemented by such a policy-instrument rule would not necessarily be the feasible path considered. Indeed, even though the policy instrument would, by construction, take the same in-equilibrium value under such a policy-instrument rule as on this path, $\mathcal{P} \mathcal{S}$ 's actions might not, as follows from Galí's (2011) analysis. ${ }^{40}$ I overcome this difficulty by designing a policy-instrument rule that is satisfied on the feasible path considered. Therefore, this path is one stationary solution of the system made of the structural equations and this rule. Since this rule ensures local equilibrium determinacy, that system has a unique stationary solution, which must then coincide with that path.

Finally, the third and last result encapsulated in Proposition 7 is that the policy-instrument rule in question is arithmetically designable, that is to say that $\mathbf{F}(L), G(L)$, and $\mathbf{H}(L)$ can be - and, in Appendix B.2, are - derived from $\mathbf{A}_{1}(L), \mathbf{A}_{2}(L), \mathbf{B}(L), \mathbf{C}(L), \mathbf{D}(L), \mathbf{S}(L)$, and $\mathbf{T}(L)$ with a finite number of arithmetic operations (addition, subtraction, multiplication, and division). In particular, the design of this rule does not require the determination of any polynomial roots (except trivially roots of polynomials of degree one), nor equivalently the determination of any matrix eigenvalues (except trivially eigenvalues of one-by-one matrices). ${ }^{41}$ Therefore, it does not require the use of any existing algorithm to solve locally linearizable dynamic stochastic discrete-time infinite-horizon rational-expectations models (e.g., the algorithms developed by Klein, 2000, and Sims, 2002). Instead, it uses Bézout's identity, the Euclidean division, and Cramer's rule, all of which involve a finite number of arithmetic operations, to directly transform the polynomials characterizing the structural equations and the implementable path considered into the polynomials characterizing the policy-instrument rule.

This arithmetic-designability property implies that, for each model and each implementable path, the coefficients of the policy-instrument rule in question can be expressed as explicit functions of the structural and implementable-path parameters. More precisely, each element of $\mathbf{F}(L), G(L)$, and $\mathbf{H}(L)$ can

\footnotetext{
${ }^{40}$ Galí (2011) shows that the multiplicity result he obtains in the context of Section 2's basic New Keynesian model (as I relate in Footnote 15) extends to Smets and Wouters' (2007) typical medium-scale DSGE model as well.

${ }^{41} \mathrm{As}$ is well known, generically speaking, polynomial roots cannot be arithmetically determined for polynomial degrees strictly higher than one. Nor can they be algebraically determined - i.e., determined with a finite number of algebraic operations (addition, subtraction, multiplication, division, exponentiation with rational exponents) - for polynomial degrees strictly higher than four, as established by the Abel-Ruffini theorem, which is named after Paolo Ruffini (1765-1822) and Niels Henrik Abel (1802-1829).
} 
be expressed as a rational function of the elements of $\mathbf{A}_{1}(L), \mathbf{A}_{2}(L), \mathbf{B}(L), \mathbf{C}(L), \mathbf{D}(L), \mathbf{S}(L)$, and $\mathbf{T}(L)$, i.e. as a fraction of polynomial functions of these elements (with real-number coefficients). ${ }^{42}$ Of course, unless the specific model and implementable path considered are particularly simple, these rational functions will involve, by most standards, an unusually large number of terms in the numerator and the denominator. However, their manipulation with a symbolic-computation software should raise no practical difficulty, for instance to compute their derivatives and determine how the coefficients of the policy-instrument rule respond to an arbitrarily small change in the value of the structural or implementable-path parameters.

The implementability condition stated in Proposition 7 is that $\mathcal{D}(X)$ should have at most $m$ unstable roots. ${ }^{43}$ How restrictive is this condition? The following proposition provides an answer to this question:

\section{Proposition 8 (a "weak Sargent-Wallace property" as a sufficient condition for Proposition} 7's implementability condition to be met): if the system of structural equations (7) has at least one stationary solution $\left(\mathbf{Y}_{t}\right)_{t \in \mathbb{Z}}$ whatever the exogenous stationary process for $\left(i_{t}\right)_{t \in \mathbb{Z}}$, then $\mathcal{D}(X)$ has at most $m$ unstable roots (taking into account their multiplicity).

Proof: suppose that the system of structural equations $(7)$ has at least one stationary solution $\left(\mathbf{Y}_{t}\right)_{t \in \mathbb{Z}}$ whatever the exogenous stationary process for $\left(i_{t}\right)_{t \in \mathbb{Z}}$. Consider the policy-instrument rule $i_{t}=\Phi_{t}$, where $\Phi_{t}$ follows an arbitrary exogenous stationary process. This rule is of type $(8)$ with $\mathbf{F}(L)=\mathbf{0}$ and $G(L)=1$, and satisfies (10) and (11). Therefore, Lemma 1 implies that (i) the system made of (7) and this rule can be written in Blanchard and Kahn's (1980) form with $m$ non-predetermined variables, and (ii) the non-zero eigenvalues of this system are (and have the same multiplicity as) the non-zero roots of (12), i.e. the non-zero roots of $\Delta_{N+1}(X)$. Since this system has - by assumption - at least one stationary solution $\left(\mathbf{Y}_{t}\right)_{t \in \mathbb{Z}}$, Blanchard and Kahn's (1980) order condition implies that $\Delta_{N+1}(X)$ has at most $m$ unstable roots (taking into account their multiplicity), and hence so does $\mathcal{D}(X)$. Proposition 8 follows.

Proposition 8 says that Proposition 7's implementability condition is met as soon as the system of structural equations $(7)$ has at least one stationary solution $\left(\mathbf{Y}_{t}\right)_{t \in \mathbb{Z}}$ whatever the exogenous stationary

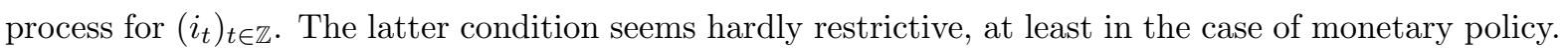
Indeed, it is well known that existing DSGE models of the monetary transmission mechanism typically have what Giannoni and Woodford (2002) and Woodford (2003a, Chapter 8) call the "Sargent-Wallace property," after Sargent and Wallace (1975), that is to say that interest-rate rules expressing the interest rate as a function of only exogenous shocks typically lead to local equilibrium multiplicity in these models. Proposition 8 therefore implies that these models typically meet Proposition 7's implementability condition. Proposition 7 then implies that all feasible paths are typically implementable in these models when $\mathcal{P} \mathcal{M}$ observes actions and shocks, possibly with lags.

That said, only particularly simple models, such as Section 2's basic New Keynesian model, have been analytically shown to have this Sargent-Wallace property for all admissible values of their structural

\footnotetext{
${ }^{42}$ In the general case, though, the coefficients of the policy-instrument rule in question cannot easily be expressed as rational functions of the structural and implementable-path parameters, because the precise number and nature of arithmetic operations involved depend on the specific model and implementable path at hand in a non-trivial way.

${ }^{43}$ This condition is sufficient, but not necessary, essentially because when $\max \left\{m_{j}^{c}-m_{j}^{b} \mid j \in J_{C}\right\} \geq 1+l_{Y}$, there may exist a rule of type (8) satisfying (10), (13), and (14), but not (11), such that the system made of the structural equations (7) and this rule has more than $m$ non-predetermined variables.
} 
parameters. ${ }^{44}$ More complex models have been checked to have this property only numerically, for some specific values of their structural parameters. Now, a formal proof that Proposition 7's implementability condition is met for all admissible values of the structural parameters would be of high value added, especially given my emphasis on arithmetically designable policy-instrument rules. Of course, such a proof can be established only on a model-specific basis. One way to establish it is to (i) use Euclid's algorithm to arithmetically derive $\mathcal{D}(X)$, and (ii) show that $\mathcal{D}(X)$ is of degree strictly lower than $m$ for all admissible values of the structural parameters (except possibly a set of values of measure zero). Another way to establish it is to (i) note that the roots of $\mathcal{D}(X)$, if any, are the solutions in $X$ of the system made of the equations $\Delta_{i}(X)=0$ for $i \in\left\{1, \ldots, N^{a}\right\} \cup\{N+1\}$, (ii) note that this system is nonlinear in the unknown $X$, but linear in the $K$ unknowns $X^{k}$ for $k \in\{1, \ldots, K\}$ with $K \equiv \max \left\{d_{\Delta_{i}(X)} \mid i \in\right.$ $\left.\left\{1, \ldots, N^{a}\right\} \cup\{N+1\}\right\}$ (where, for any $\mathcal{P}(X) \in \mathbb{R}[X], d_{\mathcal{P}}$ denotes the degree of $\mathcal{P}(X)$ ), and (iii) if $K<N^{a}+1$, then check analytically that this linear system has no solution $\left(X, X^{2}, \ldots, X^{K}\right)$, and hence that $\mathcal{D}(X)=1$, for all admissible values of the structural parameters (except possibly a set of values of measure zero). For instance, applying the latter method to Smets and Wouters' (2007) typical mediumscale DSGE model, one can easily show that $\mathcal{D}(X)=1$, and hence that Proposition 7's implementability condition is met, for all admissible values of the structural parameters of this model (except possibly a set of values of measure zero). Therefore, all feasible paths are implementable in this model, for all admissible values of its structural parameters (except possibly a set of values of measure zero), when the central bank observes actions and shocks, possibly with lags.

\subsection{When $\mathcal{P M}$ Observes Actions and Expectations, Possibly with Lags}

I now turn to the case in which $\mathcal{P} \mathcal{M}$ observes all actions, all expectations, and no shocks. In addition, I allow for the possibility that $\mathcal{P} \mathcal{M}$ observes $\mathcal{P S}$ 's actions and expectations with some lags. More specifically, I assume that there exists $l_{Y} \in \mathbb{N}$ such that $O_{t}^{\mathcal{P M}}=\left\{\mathbf{Y}^{a, t-1-l_{Y}}, i^{t-1}, \mathbb{E}^{t-1-l_{Y}}\left\{\mathbf{Y}^{a}\right\}, \mathbb{E}^{t-1-l_{Y}}\{i\}\right\}$. In this case, the rules of type (8) that are consistent with $O_{t}^{\mathcal{P M}}$ are those that satisfy

$$
\begin{aligned}
& \text { if } l_{Y} \geq 1 \text {, then } \forall k \in\left\{1, \ldots, l_{Y}\right\} \cap\left\{1, \ldots, n^{e}\right\}, \mathbf{E}_{1, k}=\mathbf{0}, \\
& \text { if } l_{Y} \geq 1 \text {, then } \forall k \in\left\{1, \ldots, l_{Y}\right\} \cap\left\{1, \ldots, n^{f}\right\}, \mathbf{F}_{k}=\mathbf{0}, \\
& \text { and } \mathbf{H}(L)=\mathbf{0},
\end{aligned}
$$

while the feasible paths, i.e. the paths of type (9) that are consistent with $O_{t}^{\mathcal{P} \mathcal{M}}$, are those that satisfy

$$
\begin{gathered}
\text { if } l_{Y} \geq 1 \text {, then } \forall k \in\left\{1, \ldots, l_{Y}\right\} \cap\left\{1, \ldots, n^{s}\right\}, \mathbf{v}_{N+1}^{\prime} \mathbf{s}_{k}\left[\begin{array}{lll}
\mathbf{v}_{1} & \ldots & \mathbf{v}_{N}
\end{array}\right]=\mathbf{0}, \\
\text { and } \mathbf{v}_{N+1}^{\prime} \mathbf{T}(L)=\mathbf{0} .
\end{gathered}
$$

I then obtain the following proposition, which generalizes Proposition 5 to any policy, any model, any observation-lag length, and any feasible path:

Proposition 9 (feasible-path implementation, in the general model, when $\mathcal{P M}$ observes actions and expectations, possibly with lags): if $\mathcal{D}(X)$ has at most $m$ unstable roots (taking into account their multiplicity), and if $\operatorname{rank}\left[\mathbf{A}_{2}(X)\right]=M_{2}$ and $\operatorname{rank}[\mathbf{D}(X)]=M_{1}$ over $\mathbb{R}[X]$, then, for any $l_{Y} \in \mathbb{N}$, for any path $\left(\mathbf{Y}_{t}, i_{t}\right)_{t \in \mathbb{Z}}$ of type (9) satisfying (22) and (23), there exists an arithmetically

\footnotetext{
${ }^{44}$ A proof that Section 2's basic New Keynesian model has this Sargent-Wallace property for all admissible values of its structural parameters can be found in, e.g., Woodford (2003a, Chapter 4).
} 
designable policy-instrument rule of type (8) satisfying (19), (20), and (21), such that this path $\left(\mathbf{Y}_{t}, i_{t}\right)_{t \in \mathbb{Z}}$ is the unique stationary solution of the system made of the structural equations (7) and that rule.

Proof: see Appendix B.3. In short, Appendix B.3 proceeds along the lines of the proof of Proposition 5 , but (i) starts from the policy-instrument rule designed by Proposition 7 when $l_{\varepsilon}=l_{Y}+1$, instead of the interest-rate rule stated in Proposition 4, and (ii) uses the condition that $\operatorname{rank}\left[\mathbf{A}_{2}(X)\right]=M_{2}$ and $\operatorname{rank}[\mathbf{D}(X)]=M_{1}$ over $\mathbb{R}[X]$, i.e. that $\forall\left(P_{1}(X), \ldots, P_{M_{2}}(X)\right) \in \mathbb{R}[X]^{M_{2}} \backslash(0, \ldots, 0), \mathbf{A}_{2}(X)\left[P_{1}(X) \quad \ldots\right.$ $\left.P_{M_{2}}(X)\right]^{\prime} \neq \mathbf{0}$, and $\forall\left(P_{1}(X), \ldots, P_{M_{1}}(X)\right) \in \mathbb{R}[X]^{M_{1}} \backslash(0, \ldots, 0), \mathbf{D}(X)\left[\begin{array}{lll}P_{1}(X) & \ldots & P_{M_{1}}(X)\end{array}\right]^{\prime} \neq \mathbf{0}$, to transform this rule into a rule involving only actions and expectations.

Proposition 9 provides, when $\mathcal{P} \mathcal{M}$ observes actions and expectations (possibly with lags), for each feasible path, two conditions that are jointly sufficient for this path to be implementable (and says that, when these conditions are met, a corresponding policy-instrument rule can be designed arithmetically). I have argued, in the previous section, that the first of these two implementability conditions (namely, that $\mathcal{D}(X)$ should have at most $m$ unstable roots) is hardly restrictive. My impression is that the second one (namely, that $\operatorname{rank}\left[\mathbf{A}_{2}(X)\right]=M_{2}$ and $\operatorname{rank}[\mathbf{D}(X)]=M_{1}$ over $\mathbb{R}[X]$ ) is no more restrictive in the absence of news shocks of the kind described in Subsection 3.3. In fact, I have been unable to find any existing DSGE model whose exogenous disturbances and structural equations (i) are of type (6) and (7) satisfying Assumptions 1 to 4, (ii) feature no news shocks of the kind described in Subsection 3.3, and (iii) do not meet this second implementability condition. Consider, for instance, Section 2's basic New Keynesian model: its exogenous disturbances and structural equations are such that

$$
\mathbf{A}_{2}(X)=\left[\begin{array}{ll}
1 & 0 \\
0 & 1
\end{array}\right] \text { and } \mathbf{D}(X)=\left[\begin{array}{cc}
1 & 0 \\
-\kappa \phi & 1
\end{array}\right]
$$

so that they trivially meet this condition. Now consider Smets and Wouters' (2007) typical medium-scale DSGE model: its exogenous disturbances and structural equations are such that

$$
\underset{(6 \times 6)}{\mathbf{A}_{2}(X)}=\left[\begin{array}{cccccc}
1 & 0 & 0 & 0 & 0 & 0 \\
0 & 1 & 0 & 0 & 0 & 0 \\
\rho_{g a} & 0 & 1 & 0 & 0 & 0 \\
0 & 0 & 0 & 1 & 0 & 0 \\
0 & 0 & 0 & 0 & 1-\mu_{p} X & 0 \\
0 & 0 & 0 & 0 & 0 & 1-\mu_{w} X
\end{array}\right] \text { and } \underset{(13 \times 6)}{\mathbf{D}(X)}=\left[\begin{array}{cccccc}
\phi_{p} & 0 & 0 & 0 & 0 & 0 \\
1 & 0 & 0 & 0 & 0 & 0 \\
0 & -c_{3} & 0 & 0 & 0 & 0 \\
0 & -1 & 0 & 0 & 0 & 0 \\
0 & 0 & 1 & 0 & 0 & 0 \\
0 & 0 & 0 & 1 & 0 & 0 \\
0 & 0 & 0 & k_{2} & 0 & 0 \\
0 & 0 & 0 & 0 & 1 & 0 \\
0 & 0 & 0 & 0 & 0 & 1 \\
\hline & & 0 & &
\end{array}\right],
$$

where $\left(c_{3}, k_{2}, \mu_{p}, \mu_{w}, \phi_{p}, \rho_{g a}\right) \in \mathbb{R}^{6}$, so that they also meet this condition. ${ }^{45}$ Given the discussion about the first implementability condition in the previous subsection, Proposition 9 therefore implies that all feasible paths are implementable in these two models, for all admissible values of their structural parameters (except possibly a set of values of measure zero), when $\mathcal{P} \mathcal{M}$ observes actions and expectations, possibly with lags. However, models with news shocks of the kind described in Subsection 3.3, such as Christiano, Motto, and Rostagno's (2013), typically do not meet this second implementability condition as they are typically such that $M_{1}<M_{2}$ and hence $\operatorname{rank}\left[\mathbf{A}_{2}(X)\right]<M_{2}$ over $\mathbb{R}[X]$ (as clear from the example given in Subsection 3.3).

\footnotetext{
${ }^{45}$ In this model, $\mathbf{A}_{2}(L) \neq \mathbf{A}_{2}(0)$ because the price-markup and wage-markup disturbances follow ARMA $(1,1)$ processes, and $\mathbf{A}_{2}(0)$ is not diagonal because the spending shock is a linear combination of the productivity shock and another white noise.
} 


\subsection{When $\mathcal{P} \mathcal{M}$ Observes Actions, Possibly with Lags}

I finally consider the case in which $\mathcal{P} \mathcal{M}$ observes all actions, no expectations, and no shocks. In addition, I allow again for the possibility that $\mathcal{P} \mathcal{M}$ observes $\mathcal{P S}$ 's actions with some lags. More specifically, I assume that there exists $l_{Y} \in \mathbb{N}$ such that $O_{t}^{\mathcal{P M}}=\left\{\mathbf{Y}^{a, t-1-l_{Y}}, i^{t-1}\right\}$. In this case, the rules of type (8) that are consistent with $O_{t}^{\mathcal{P} M}$ are those that satisfy (10), (13), (14) and (21), while the feasible paths, i.e. the paths of type (9) that are consistent with $O_{t}^{\mathcal{P}}$, are those that satisfy $(16),(17)$, and (23). I then obtain the following proposition, which generalizes Proposition 6 to any policy, any model, any observation-lag length, and any feasible path:

Proposition 10 (feasible-path implementation, in the general model, when $\mathcal{P} \mathcal{M}$ observes actions, possibly with lags): for any $l_{Y} \in \mathbb{N}$, for any path $\left(\mathbf{Y}_{t}, i_{t}\right)_{t \in \mathbb{Z}}$ of type (9) satisfying (16), (17), and (23), if $\operatorname{rank}\left\{\mathbf{T}(X)^{\prime}\left[\begin{array}{lll}\mathbf{v}_{1} & \ldots & \mathbf{v}_{N^{a}}\end{array}\right]\right\}=N^{a}$ over $\mathbb{R}[X]$, then the following two statements are equivalent to each other:

(a) there exists a policy-instrument rule of type (8) satisfying (10), (13), (14), and (21), such that this path $\left(\mathbf{Y}_{t}, i_{t}\right)_{t \in \mathbb{Z}}$ is the unique stationary solution of the system made of the structural equations (7) and that rule,

(b) the system made of the structural equations (7) and the last line of (9) has a unique stationary solution.

Proof: (b) trivially implies (a). To show that (a) implies (b), consider a given path of type (9) satisfying (17) and (23). The condition that $\operatorname{rank}\left\{\mathbf{T}(X)^{\prime}\left[\begin{array}{lll}\mathbf{v}_{1} & \ldots & \mathbf{v}_{N^{a}}\end{array}\right]\right\}=N^{a}$ over $\mathbb{R}[X]$ (i.e. that $\left.\forall\left(P_{1}(X), \ldots, P_{N^{a}}(X)\right) \in \mathbb{R}[X]^{N^{a}} \backslash(0, \ldots, 0), \sum_{j=0}^{N^{a}} P_{j}(X) \mathbf{T}(X)^{\prime} \mathbf{v}_{j} \neq \mathbf{0}\right)$ is necessary and sufficient for the system made of the first $N^{a}$ lines of (9), taken at all dates $t \in \mathbb{Z}$, to imply no equation without exogenous shocks. Therefore, under this condition, any rule of type (8) that (i) satisfies (10), (13), (14), and (21), and (ii) is consistent with the path considered, is necessarily of type

$$
K(L) \mathbf{v}_{N+1}^{\prime} \mathbf{S}(L)\left[\begin{array}{ll}
\mathbf{Y}_{t}^{\prime} & i_{t}
\end{array}\right]^{\prime}=0
$$

with $K(L) \equiv \sum_{j=0}^{n^{k}} k_{j} L^{j}$, where $n^{k} \in \mathbb{N}$, all $k_{j}$ are real numbers and, without any loss in generality, $k_{0}$ is normalized to one. Now, for any $K(L),(24)$ has a unique stationary solution in $\left(\mathbf{v}_{N+1}^{\prime} \mathbf{S}(L)\left[\mathbf{Y}_{t}^{\prime} \quad i_{t}\right]^{\prime}\right)_{t \in \mathbb{Z}}$, namely $\mathbf{v}_{N+1}^{\prime} \mathbf{S}(L)\left[\begin{array}{cc}\mathbf{Y}_{t}^{\prime} & i_{t}\end{array}\right]^{\prime}=0$, which corresponds to the last line of $(9)$. Therefore, for any $K(L)$, the system made of the structural equations (7) and the rule (24) has exactly the same stationary solutions in $\left(\mathbf{Y}_{t}, i_{t}\right)_{t \in \mathbb{Z}}$ as the system made of the structural equations (7) and the last line of (9). Proposition 10 follows.

An alternative way to understand this result is to note that, according to a standard result of timeseries analysis (see, e.g., Hamilton, 1994, Chapter 10, Proposition 10.1), for any $K(L)$, the eigenvalues of the system made of the structural equations (7) and the rule (24) are those of the system made of the structural equations $(7)$ and the last line of (9), plus the roots of polynomial $X^{n^{k}} K\left(X^{-1}\right)$. Thus, choosing some $K(L)$ amounts to choose some additional eigenvalues for the system without changing the number of its non-predetermined variables. If the system made of the structural equations (7) and the last line of (9) has strictly fewer non-predetermined variables than unstable eigenvalues, then no choice of $K(L)$ can make the resulting system meet Blanchard and Kahn's (1980) order condition. Alternatively, if the system made of the structural equations (7) and the last line of (9) has strictly more 
non-predetermined variables than unstable eigenvalues, then $K(L)$ can be chosen such that the resulting system satisfies Blanchard and Kahn's (1980) order condition, but this system will not meet Blanchard and Kahn's (1980) rank condition, because the additional unstable eigenvalues will not have the right eigenvectors.

Proposition 10 provides, when $\mathcal{P M}$ observes actions (possibly with lags), for each feasible path of a certain kind, a necessary and sufficient condition for this path to be implementable (in which case a corresponding policy-instrument rule is directly provided by the last line of the feasible path). The restriction on the kind of feasible paths considered (namely, that $\operatorname{rank}\left\{\mathbf{T}(X)^{\prime}\left[\begin{array}{lll}\mathbf{v}_{1} & \ldots & \mathbf{v}_{N^{a}}\end{array}\right]\right\}=N^{a}$ over $\mathbb{R}[X])$ requires that $M_{2} \geq N^{a}$, but is consistent with $M_{2}=N^{a}$. In the latter case, since $\mathcal{P} \mathcal{M}$ 's observation set includes $N^{a}+1$ actions ( $N^{a}$ actions of $\mathcal{P S}$, plus her own action), there is one more endogenous variable in $\mathcal{P} \mathcal{M}$ 's observation set than exogenous shocks in the model, so that there is one degree of freedom in the choice of a policy-instrument rule consistent with $\mathcal{P} \mathcal{M}$ 's observation set and the feasible path considered. It might be thought at first sight that this degree of freedom could be successfully exploited to find a policy-instrument rule that is consistent with $\mathcal{P} \mathcal{M}$ 's observation set and implements the feasible path considered as the unique local equilibrium. Proposition 10 shows that it cannot, and that the feasible path considered is not implementable when the policy-instrument rule corresponding to the last line of its VARMA representation (9) does not ensure local equilibrium determinacy. One example of feasible path that may not be implementable when $\mathcal{P} \mathcal{M}$ observes past actions is the timeless-perspective Ramsey-optimal feasible path. I have found in Subsection 2.7 that, in the basic New Keynesian model, this path seems to be non-implementable only for empirically implausible calibrations of that model. I will soon show in Subsection 5.1 that, in Svensson and Woodford's (2005) New Keynesian model with policy transmission lags, this path is not implementable for empirically relevant calibrations of that model.

\section{$5 \quad$ Applications and Implications}

In this section, I apply the general results of the previous section to some specific models, policy instruments, observation sets, and feasible paths. More precisely, for each model, policy instrument, and path considered, I check whether the conditions for this path to be implementable are met for empirically plausible policymaker's observation sets in that model. The outcome of this check may have interesting implications, which I highlight in due course.

\subsection{Optimal Monetary Policy}

I start by considering monetary policy and the timeless-perspective Ramsey-optimal feasible path as in Section 2, but no longer in the context of the basic New Keynesian model. Some fifteen years ago, Rotemberg and Woodford (1999, p. 103) wrote that "the construction of a feedback rule for the funds rate that implements the optimal allocation - that is not only consistent with it but also renders it the unique stationary equilibrium consistent with the proposed policy rule - remains a nontrivial problem." In essence, this statement is still valid today when the interest-rate rule is required to be explicit, i.e. when the central bank's $(\mathcal{C B})$ observation set does not include the private sector's $(\mathcal{P S})$ current actions and expectations. Indeed, as already mentioned in the introduction, to my knowledge, only Svensson and Woodford (2005, Subsections 3.6 and 4.2, and Section 5) manage to design, in a 
model with a forward-looking system of structural equations, explicit interest-rate rules that implement the timeless-perspective Ramsey-optimal feasible path as the unique local equilibrium for all admissible values of the structural parameters. ${ }^{46}$

The model considered by Svensson and Woodford (2005) is Section 2's basic New Keynesian model with one-period policy transmission lags and $\mathrm{AR}(1)$ disturbances. In this model, at each date $t, \mathcal{P S}$ sets the inflation rate $\pi_{t}$ and the welfare-relevant output gap $x_{t}$ according to the following IS equation and Phillips curve:

$$
\begin{aligned}
& x_{t}=\mathbb{E}_{t-1}\left\{x_{t+1}\right\}-\sigma\left(\mathbb{E}_{t-1}\left\{i_{t}\right\}-\mathbb{E}_{t-1}\left\{\pi_{t+1}\right\}-r_{t}^{n}\right) \\
& \pi_{t}=\beta \mathbb{E}_{t-1}\left\{\pi_{t+1}\right\}+\kappa \mathbb{E}_{t-1}\left\{x_{t}\right\}+u_{t}
\end{aligned}
$$

where $\beta, \kappa, \sigma$ are such that $0<\beta<1, \kappa>0, \sigma>0$, and $i_{t}$ denotes the interest rate set by $\mathcal{C B}$ at date $t$. The exogenous disturbances $r_{t}^{n}$ and $u_{t}$ follow the $\mathrm{AR}(1)$ processes characterized by

$$
\begin{aligned}
& r_{t}^{n}=\omega r_{t-1}^{n}+\eta_{t}, \\
& u_{t}=\rho u_{t-1}+\epsilon_{t},
\end{aligned}
$$

where $\omega$ and $\rho$ are such that $0 \leq \omega<1$ and $0 \leq \rho<1$, and $\eta_{t}$ and $\epsilon_{t}$ are i.i.d. exogenous shocks such that $\mathbb{E}\left\{\eta_{t} \epsilon_{t-k}\right\}=0$ for any $k \in \mathbb{Z} .{ }^{47}$ Finally, the date- $t$ welfare loss function is of the form $L_{t}=\mathbb{E}_{t}\left\{\sum_{k=0}^{+\infty} \beta^{k}\left[\left(\pi_{t+k}\right)^{2}+\lambda\left(x_{t+k}\right)^{2}\right]\right\}$, where $\lambda>0$. Svensson and Woodford (2005) determine the timeless-perspective Ramsey-optimal feasible path when $\mathcal{C B}$ 's observation set is such that $\mathcal{C B}$ 's observation constraint is slack in the optimization programme defining this path. ${ }^{48}$ They design three explicit interest-rate rules that implement this path as the unique local equilibrium for all admissible values of the structural parameters. All three rules involve past expectations of $\mathcal{P} \mathcal{S}$ and past exogenous disturbances. ${ }^{49}$ However, it seems reasonable, in the context of this model, to consider that $\mathcal{C B}$ may not observe $\mathcal{P} \mathcal{S}$ 's expectations or exogenous shocks. ${ }^{50}$ Therefore, the question arises as to whether the timeless-perspective Ramsey-optimal feasible path is still implementable in this model when $\mathcal{C B}$ observes only past actions and shocks, or only past actions and expectations, or only past actions.

This question cannot be addressed by using Svensson and Woodford's (2005) algebraic method of designing interest-rate rules, which is similar to the guess-and-verify method that I use in Section 2 , for the reason discussed in Subsection 2.8 (namely, because the degree of the characteristic polynomials of the systems to be considered would be too large for the computations to be analytically tractable). It can easily be answered, however, by applying the general results of the previous section, as I now do. Let me first rewrite the IS equation (25) and Phillips curve (26) in terms of the output level $y_{t}$, instead of the welfarerelevant output gap $x_{t}$, as it seems relevant to assume that $\mathcal{C B}$ directly observes the former, not the latter.

\footnotetext{
${ }^{46}$ I qualify a system of structural equations of type (7) satisfying Assumptions 1 to 4 as "forward-looking" when $\exists j \in$ $\{1, \ldots, N\}, m_{j}^{b}>0$, or $\exists j \in J_{C}, m_{j}^{c}>0$ (so that, due to Assumption 3, it cannot be rewritten in an equivalent form with $\forall j \in\{1, \ldots, N\}, m_{j}^{b} \leq 0$, and $\left.\forall j \in J_{C}, m_{j}^{c} \leq 0\right)$. Focusing on models with a forward-looking system of structural equations does not entail any loss in generality since it is only in these models that the policymaker faces a non-trivial local-equilibrium-indeterminacy problem. The set of such models includes, of course, most models built on microeconomic foundations.

${ }^{47}$ In Svensson and Woodford's (2005) original model, the mean $\bar{r}$ of $r_{t}^{n}$ is allowed to be non-zero. For simplicity and without any loss in generality, I set $\bar{r}=0$.

${ }^{48}$ Given that $i_{t}$ appears in the IS equation (25) and Phillips curve (26) only through the term $\mathbb{E}_{t-1}\left\{i_{t}\right\}$, such an observation set need not include any current endogenous or exogenous variable.

${ }^{49}$ The first rule involves $\mathbb{E}_{t-1}\left\{\pi_{t}\right\}, \mathbb{E}_{t-1}\left\{x_{t}\right\}, r_{t-1}^{n}, u^{t-1}$ (Subsection 3.6), the second one $\mathbb{E}_{t-1}\left\{\pi_{t}\right\}, \mathbb{E}_{t-1}\left\{x_{t}\right\}$, $\mathbb{E}_{t-2}\left\{x_{t-1}\right\}, r_{t-1}^{n}, u_{t-1}$ (Subsection 4.2), and the third one $\mathbb{E}_{t-1}\left\{\pi_{t}\right\}, \mathbb{E}_{t-1}\left\{x_{t}\right\}, \mathbb{E}_{t-2}\left\{x_{t-1}\right\}, r_{t-1}^{n}, u^{t-1}$ (Section 5 ).

${ }^{50}$ In this model, as mentioned in Svensson and Woodford (2005), $r_{t}^{n}$ may come from exogenous variations in a component of aggregate expenditure other than (predetermined) private consumption, and $u_{t}$ from exogenous measurement errors in the price index or exogenous variations in the markup of (non-predetermined) retail prices over (predetermined) wholesale prices.
} 
To that aim, consider exogenous variations in a component of aggregate expenditure other than private consumption, e.g. government purchases, and note $\nu_{t}$ their realization at date $t$. Following Woodford (2003a, Chapters 4 and 5), I get that $y_{t}=\mathbb{E}_{t-1}\left\{y_{t+1}\right\}-\sigma\left(\mathbb{E}_{t-1}\left\{i_{t}\right\}-\mathbb{E}_{t-1}\left\{\pi_{t+1}\right\}\right)+\nu_{t}-\mathbb{E}_{t-1}\left\{\nu_{t+1}\right\}$ and $x_{t}=y_{t}-\phi \nu_{t}$, where $0<\phi<1$. These two equations are consistent with the IS equation (25) and the $\operatorname{AR}(1)$ process (27) if $\nu_{t}$ follows the stationary $\operatorname{ARMA}(1,1)$ process characterized by $\nu_{t}=\omega \nu_{t-1}+\frac{\sigma}{1-\phi} \epsilon_{t}+\frac{\sigma \omega^{2}}{(1-\phi)(1-\omega)} \epsilon_{t-1}$. In this case, $\nu_{t}$ can be rewritten as $\nu_{t}=\frac{\sigma}{1-\phi} r_{t}^{n}+\frac{\sigma \omega^{2}}{(1-\phi)(1-\omega)} r_{t-1}^{n}$, and the IS equation (25) and Phillips curve (26) as

$$
\begin{aligned}
y_{t} & =\mathbb{E}_{t-1}\left\{y_{t+1}\right\}-\sigma\left(\mathbb{E}_{t-1}\left\{i_{t}\right\}-\mathbb{E}_{t-1}\left\{\pi_{t+1}\right\}-\frac{r_{t}^{n}}{1-\phi}\right) \\
\pi_{t} & =\beta \mathbb{E}_{t-1}\left\{\pi_{t+1}\right\}+\kappa \mathbb{E}_{t-1}\left\{y_{t}\right\}-\frac{\kappa \phi \sigma \omega}{(1-\phi)(1-\omega)} r_{t-1}^{n}+u_{t} .
\end{aligned}
$$

Then, the system made of (27), (28), (29), and (30) can easily be rewritten in a form of type (6) and (7) with $M_{1}=M_{2}=N^{a}=2, N^{e}=5, \boldsymbol{\xi}_{t}=\left[\begin{array}{ll}r_{t}^{n} & u_{t}\end{array}\right]^{\prime}, \varepsilon_{t}=\left[\begin{array}{ll}\eta_{t} & \epsilon_{t}\end{array}\right]^{\prime}, \mathbf{Y}_{t}^{a}=\left[\begin{array}{ll}\pi_{t} & y_{t}\end{array}\right]^{\prime}$, $\mathbf{Y}_{t}^{e}=\left[\begin{array}{lllll}\mathbb{E}_{t}\left\{\pi_{t+1}\right\} & \mathbb{E}_{t}\left\{\pi_{t+2}\right\} & \mathbb{E}_{t}\left\{y_{t+1}\right\} & \mathbb{E}_{t}\left\{y_{t+2}\right\} & \mathbb{E}_{t}\left\{i_{t+1}\right\}\end{array}\right]^{\prime}$,

$$
\begin{aligned}
& \mathbf{A}_{1}(L)=\left[\begin{array}{cc}
1-\omega L & 0 \\
0 & 1-\rho L
\end{array}\right], \quad \mathbf{A}_{2}(L)=\left[\begin{array}{ll}
1 & 0 \\
0 & 1
\end{array}\right], \quad \mathbf{C}(L)=\left[\begin{array}{lllllll}
0 & 0 & 0 & 0 & 0 & 0 & -L^{-1}
\end{array}\right]^{\prime},
\end{aligned}
$$

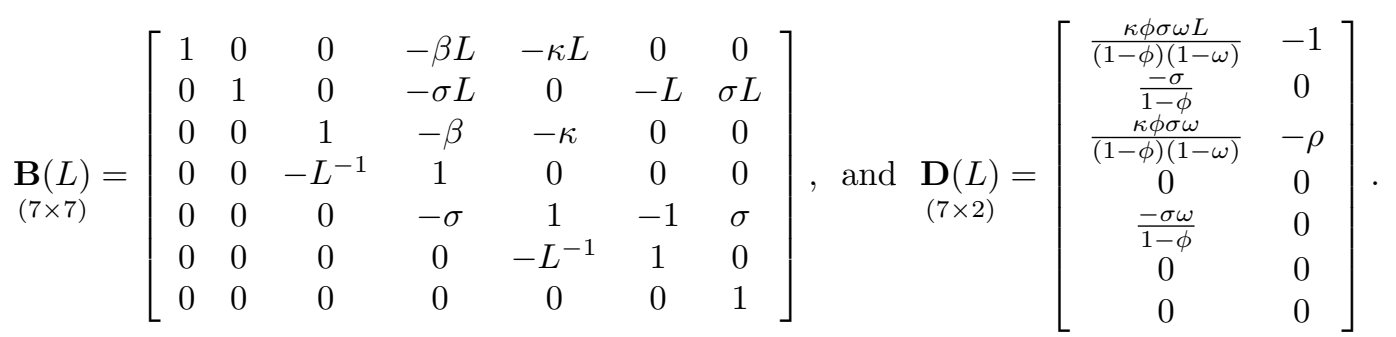

Moreover, it is easy to check that $\mathbf{B}(L)$ and $\mathbf{C}(L)$ satisfy Assumptions 1 to 4 for all admissible values of the structural parameters $\beta, \kappa, \phi, \rho, \sigma$, and $\omega$. Finally, it is also easy to check that (i) $\Delta_{1}(X)=\kappa \sigma X^{2}$, which implies that $\mathcal{D}(X)$ has no unstable roots, and (ii) $\operatorname{rank}\left[\mathbf{A}_{2}(X)\right]=M_{2}$ and $\operatorname{rank}[\mathbf{D}(X)]=M_{1}$ over $\mathbb{R}[X]$. Therefore, Svensson and Woodford's (2005) model meets Propositions 7 and 9's implementability conditions for all admissible values of its structural parameters. As a consequence, all feasible paths, in particular the timeless-perspective Ramsey-optimal feasible path, are implementable in this model, for all admissible values of its structural parameters, when $\mathcal{C B}$ observes past actions and shocks, or past actions and expectations, possibly with lags.

In order to determine whether the timeless-perspective Ramsey-optimal feasible path is also implementable in this model when $\mathcal{C B}$ observes only past actions (without lags), I first need to write this path in a form of type (9) satisfying (16) and (23). The following proposition addresses this need, focusing for expositional clarity on $\left[\begin{array}{ll}\mathbf{Y}_{t}^{a \prime} & i_{t}\end{array}\right]^{\prime}$, i.e. leaving aside $\mathbf{Y}_{t}^{e}$ :

Proposition 11 (timeless-perspective Ramsey-optimal feasible path, in Svensson and Woodford's (2005) model, when $\mathcal{C B}$ observes past actions): in Svensson and Woodford's (2005) model, there exists $\left(\alpha_{j}^{\pi}, \alpha_{j}^{y}, \alpha_{j}^{i}\right)_{1 \leq j \leq 3} \in \mathbb{R}^{9}$ such that, when $O_{t}^{\mathcal{C B}}=\left\{\pi^{t-1}, y^{t-1}, i^{t-1}\right\}$, the timeless-perspective 
Ramsey-optimal feasible path can be written in the following VARMA form:

$$
\begin{aligned}
& {\left[\begin{array}{l}
\pi_{t} \\
y_{t} \\
i_{t}
\end{array}\right]=\left[\begin{array}{ccc}
\rho+\mu & 0 & 0 \\
0 & \rho+\omega+\mu & 0 \\
\alpha_{1}^{\pi} & \alpha_{1}^{y} & \alpha_{1}^{i}
\end{array}\right]\left[\begin{array}{c}
\pi_{t-1} \\
y_{t-1} \\
i_{t-1}
\end{array}\right]+\left[\begin{array}{ccc}
-\rho \mu & 0 & 0 \\
0 & -\rho \omega-\rho \mu-\omega \mu & 0 \\
\alpha_{2}^{\pi} & \alpha_{2}^{y} & \alpha_{2}^{i}
\end{array}\right]\left[\begin{array}{c}
\pi_{t-2} \\
y_{t-2} \\
i_{t-2}
\end{array}\right]+\left[\begin{array}{c}
0 \\
0 \\
\alpha_{3}^{\pi}
\end{array}\right.} \\
& \left.\begin{array}{cc}
0 \omega \mu & 0 \\
\alpha_{3}^{y} & \alpha_{3}^{i}
\end{array}\right]\left[\begin{array}{c}
\pi_{t-3} \\
y_{t-3} \\
i_{t-3}
\end{array}\right]+\left[\begin{array}{cc}
0 & 1 \\
\frac{\sigma}{1-\phi} & 0 \\
0 & 0
\end{array}\right]\left[\begin{array}{l}
\eta_{t} \\
\epsilon_{t}
\end{array}\right]+\left[\begin{array}{cc}
0 & \frac{\rho \mu}{1-\beta \rho \mu}-\rho-\mu \\
\frac{-(\rho+\mu) \sigma}{1-\phi}-\frac{(1-\phi-\omega) \sigma \omega}{(1-\phi)(1-\omega)} & \frac{-\kappa \rho \mu}{\lambda(1-\beta \rho \mu)} \\
0
\end{array}\right]\left[\begin{array}{c}
\eta_{t-1} \\
\epsilon_{t-1}
\end{array}\right] \\
& +\left[\begin{array}{cc}
0 & \frac{-\beta \rho^{2} \mu^{2}}{1-\beta \rho \mu} \\
\frac{\rho \sigma \mu}{1-\phi}+\frac{(1-\phi-\omega)(\rho+\mu) \sigma \omega}{(1-\phi)(1-\omega)} & \frac{\kappa \rho \omega \mu}{\lambda(1-\beta \rho \mu)} \\
0 & 0
\end{array}\right]\left[\begin{array}{c}
\eta_{t-2} \\
\epsilon_{t-2}
\end{array}\right]+\left[\begin{array}{cc}
0 & 0 \\
\frac{-(1-\phi-\omega) \rho \sigma \omega \mu}{(1-\phi)(1-\omega)} & 0 \\
0 & 0
\end{array}\right]\left[\begin{array}{c}
\eta_{t-3} \\
\epsilon_{t-3}
\end{array}\right] \text {, }
\end{aligned}
$$

where $\left.\mu \equiv \frac{\lambda+\beta \lambda+\kappa^{2}-\sqrt{\left(\lambda+\beta \lambda+\kappa^{2}\right)^{2}-4 \beta \lambda^{2}}}{2 \beta \lambda} \in\right] 0 ; 1[$.

Proof: see Appendix A.5. In short, Appendix A.5 simply rewrites the timeless-perspective Ramseyoptimal feasible path determined by Svensson and Woodford (2005) in a VARMA form whose $\pi_{t}$ and $y_{t}$ lines involve no $\mathcal{P} \mathcal{S}$ 's expectations, and whose $i_{t}$ line involves no $\mathcal{P} \mathcal{S}$ 's expectations and no exogenous shocks.

The $2 \times 2$ matrix obtained by removing its last line from the $3 \times 2$ matrix multiplying [ $\left.\eta_{t} \quad \epsilon_{t}\right]^{\prime}$ in (31) is of rank two for all admissible values of the structural parameters. Therefore, any path of type (9) whose $\mathbf{Y}_{t}^{a}$ and $i_{t}$ lines are written as in (31) is such that $\operatorname{rank}\left\{\mathbf{T}(X)^{\prime}\left[\begin{array}{lll}\mathbf{v}_{1} & \ldots & \mathbf{v}_{N^{a}}\end{array}\right\}=N^{a}\right.$ over $\mathbb{R}[X]$. Proposition 10 then implies that the timeless-perspective Ramsey-optimal feasible path is implementable when $O_{t}^{\mathcal{C B}}=\left\{\pi^{t-1}, y^{t-1}, i^{t-1}\right\}$ if and only if the system made of (29), (30), and the last line of (31) has a unique stationary solution. For any calibration of the model's structural parameters, I can determine numerically, using the algebraic expression of $\left(\alpha_{j}^{\pi}, \alpha_{j}^{y}, \alpha_{j}^{i}\right)_{1 \leq j \leq 3}$ provided in Appendix A.5, whether this necessary and sufficient implementability condition is met. Since Svensson and Woodford (2005) do not calibrate their model, I set the parameters $\beta, \kappa, \lambda, \phi$, and $\sigma$ to their values in Galí's (2008, Chapter 3) or Woodford's (2003a, Chapter 4) calibration of the basic New Keynesian model described in Table 1, with $\mathbb{1}_{c l}=1$, and I consider a grid of values between zero and one for the parameters $\rho$ and $\omega$. The results that I obtain are presented in Figure 1, which shows that, in Svensson and Woodford's (2005) model, there exist admissible and empirically relevant values for the structural parameters such that the timeless-perspective Ramsey-optimal feasible path is not implementable when $\mathcal{C B}$ observes only past actions (without lags).

\subsection{Monetary Policy Based on Constant-Interest-Rate Forecasts}

A number of central banks in the world take their interest-rate decisions on the basis of macroeconomic projections that are conditional on the interest rate being constant during the projection period. Leitemo (2003) has formalized such a decision procedure by considering that, at each date, the central bank sets the interest rate at a value such that the forecast of the inflation rate, at a given horizon and conditional on the interest rate remaining at this value during the projection period, equals a given exogenous target. Leitemo (2003) shows, in a general model with a forward-looking system of structural equations, that this decision procedure can be represented by an implicit interest-rate rule. In the case where this rule ensures local equilibrium determinacy, let me define the constant-interest-rate forecast targeting 
Figure 1: Implementability of the timeless-perspective Ramsey-optimal feasible path in Svensson and Woodford's (2005) model when $\mathcal{C B}$ observes past actions

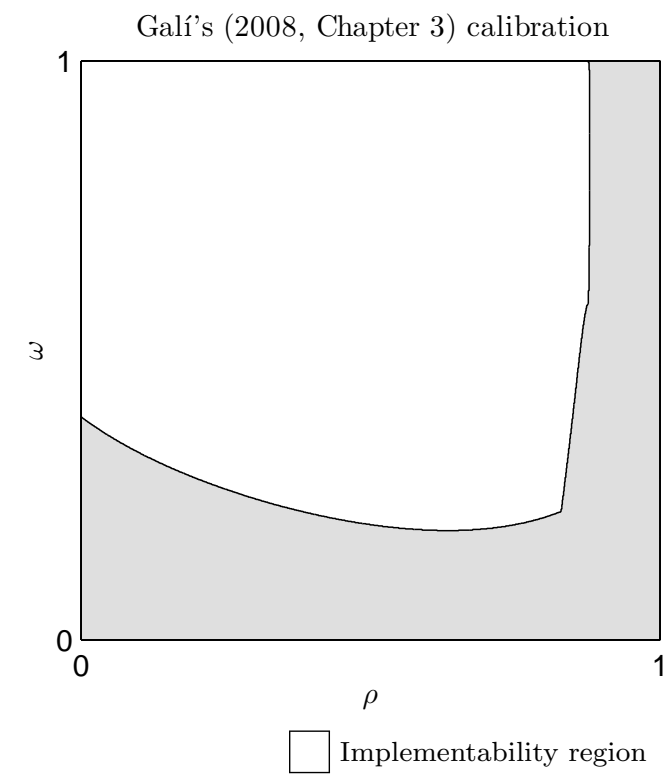

Woodford's (2003a, Chapter 4) calibration

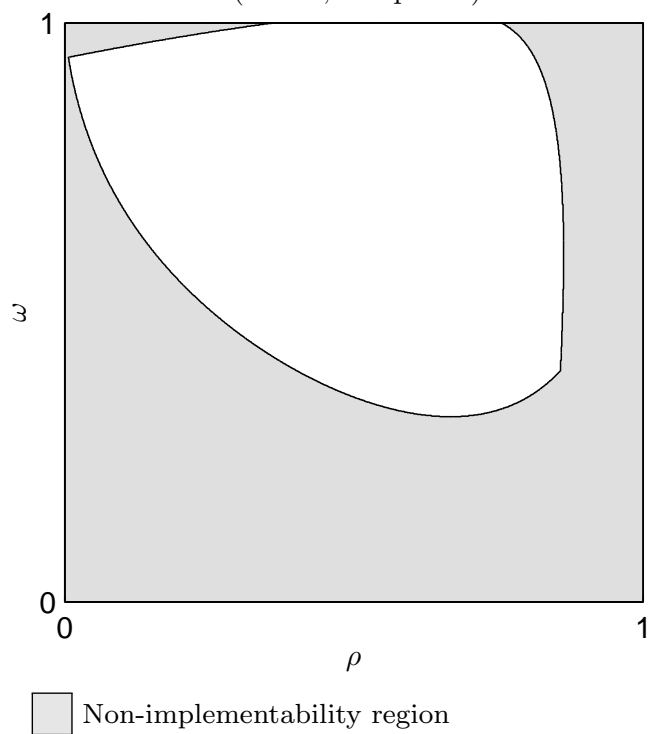

path as the unique local equilibrium under this rule. Does there exist an explicit interest-rate rule that implements this path as the unique local equilibrium?

To my knowledge, only Leitemo (2003) addresses this question in a model with a forward-looking system of structural equations. He considers a model with policy transmission lags in which, at each date $t, \mathcal{P} \mathcal{S}$ sets the inflation rate $\pi_{t}$ and the output gap $x_{t}$ according to the following structural equations:

$$
\begin{aligned}
& x_{t}=\rho_{0} x_{t-1}+\rho_{1} x_{t-2}-\beta \sum_{j=1}^{4}\left(i_{t-j}-\pi_{t-j}\right)+\eta_{t}, \\
& \pi_{t}=\alpha \mathbb{E}_{t-1}\left\{\pi_{t+1}\right\}+(1-\alpha) \pi_{t-1}+\gamma x_{t-1}+\epsilon_{t},
\end{aligned}
$$

where $\left(\alpha, \beta, \gamma, \rho_{0}, \rho_{1}\right) \in \mathbb{R}^{5}, i_{t}$ denotes the interest rate set by $\mathcal{C B}$ at date $t$, and $\eta_{t}$ and $\epsilon_{t}$ are i.i.d. exogenous shocks such that $\mathbb{E}\left\{\eta_{t} \epsilon_{t-k}\right\}=0$ for any $k \in \mathbb{Z} .^{51}$ He numerically obtains an explicit interestrate rule that is consistent with the constant-interest-rate forecast targeting path by solving, for $i_{t}$, the system made of (i) the structural equations (32) and (33) taken at dates $t+k$ for $k \geq 0$, (ii) the implicit interest-rate rule mentioned above, taken at dates $t+k$ for $k \geq 0$, and (iii) the stationarity (i.e., localness) restriction. This rule involves past actions, past expectations, and current shocks. ${ }^{52}$ However, Leitemo (2003) finds that, for the specific structural-parameter values that he considers, this rule fails to ensure local equilibrium determinacy, i.e. fails to implement that path as the unique local equilibrium. He concludes by warning against basing monetary policy on constant-interest-rate forecasts.

However, the fact that this explicit interest-rate rule fails to ensure local equilibrium determinacy does not imply that all the explicit interest-rate rules that are consistent with the constant-interest-rate forecast targeting path fail to ensure local equilibrium determinacy. So the question remains open as to whether this path is implementable in that model, for the specific structural-parameter values considered by Leitemo (2003) or for any other admissible structural-parameter values. This question can easily be

\footnotetext{
${ }^{51}$ Leitemo (2003) uses the notation $y_{t}$ to denote the output gap at date $t$. For reasons of consistency with the notations of Section 2 and Subsection 5.1, I use instead the notation $x_{t}$ for this variable, and $y_{t}$ for the output level at date $t$.

${ }^{52}$ Leitemo (2003) writes this rule in a form that is not of type (8) as it involves $\left(\pi_{t-j}\right)_{0 \leq j \leq 3},\left(x_{t-j}\right)_{0 \leq j \leq 1}$, and $\left(i_{t-j}\right)_{1 \leq j \leq 3}$. Using (32) and (33), one can straightforwardly rewrite it in a form of type (8) that involves $\left(\pi_{t-j}\right)_{1 \leq j \leq 4}$, $\left(x_{t-j}\right)_{1 \leq j \leq 2},\left(i_{t-j}\right)_{1 \leq j \leq 4}, \mathbb{E}_{t-1}\left\{\pi_{t+1}\right\}, \eta_{t}$, and $\epsilon_{t}$.
} 
answered by applying the general results of the previous section, as I now do. As it seems relevant to assume that $\mathcal{C B}$ directly observes the output level, not the output gap, let me first note $y_{t}$ the output level at date $t$ and postulate, without any loss in generality, a relationship between $x_{t}$ and $y_{t}$ of type $x_{t}=y_{t}+\delta_{\eta}(L) \eta_{t}+\delta_{\epsilon}(L) \epsilon_{t}$, where $\left(\delta_{\eta}(X), \delta_{\epsilon}(X)\right) \in \mathbb{R}[X]^{2}{ }^{53}$ Then, the system made of (32) and (33) can easily be rewritten in a form of type (6) and (7) with $M_{1}=M_{2}=N^{a}=N^{e}=2, \boldsymbol{\xi}_{t}=\varepsilon_{t}=\left[\begin{array}{lll}\eta_{t} & \epsilon_{t}\end{array}\right]^{\prime}$, $\mathbf{Y}_{t}^{a}=\left[\begin{array}{ll}\pi_{t} & y_{t}\end{array}\right]^{\prime}, \mathbf{Y}_{t}^{e}=\left[\begin{array}{ll}\mathbb{E}_{t}\left\{\pi_{t+1}\right\} & \mathbb{E}_{t}\left\{\pi_{t+2}\right\}\end{array}\right]^{\prime}, \mathbf{A}_{1}(L)=\mathbf{A}_{2}(L)=\mathbf{I}_{2}$

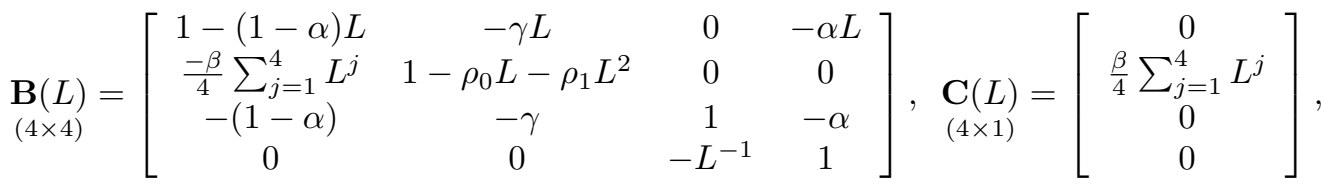

$$
\begin{aligned}
& \text { and } \underset{(4 \times 2)}{\mathbf{D}(L)}=\left[\begin{array}{cc}
-\gamma L \delta_{\eta}(L) & -1-\gamma L \delta_{\epsilon}(L) \\
-1+\left(1-\rho_{0} L-\rho_{1} L^{2}\right) \delta_{\eta}(L) & \left(1-\rho_{0} L-\rho_{1} L^{2}\right) \delta_{\epsilon}(L) \\
-\gamma \delta_{\eta}(L) & -\gamma \delta_{\epsilon}(L) \\
0 & 0
\end{array}\right]
\end{aligned}
$$

It is easy to check that $\mathbf{B}(L)$ and $\mathbf{C}(L)$ satisfy Assumptions 1 to 4 for all admissible values of the structural parameters $\alpha, \beta, \gamma, \rho_{0}$, and $\rho_{1}$, except when $\alpha=0$. In the latter case, the system of structural equations is not forward-looking, so that all feasible paths are trivially implementable for any $\mathcal{C} B$ 's observation set. Let me focus, in the following, on the alternative case in which $\alpha \neq 0$. It is also easy to check that (i)

$$
\begin{aligned}
& \Delta_{1}(X)=\frac{-\beta \gamma}{4}(X+1)(X-i)(X+i) P_{\alpha}(X), \\
& \Delta_{2}(X)=\frac{-\beta}{4}(X+1)(X-i)(X+i) Q_{\alpha}(X), \\
& \Delta_{5}(X)=\Delta_{1}(X)-X^{2}\left(X^{2}-\rho_{0} X-\rho_{1}\right) Q_{\alpha}(X),
\end{aligned}
$$

where $P_{\alpha}(X) \equiv \alpha X^{2}-\alpha X+1$ and $Q_{\alpha}(X) \equiv \alpha(2-\alpha) X^{2}-\left(1+\alpha-\alpha^{2}\right) X+(1-\alpha)$, (ii) $\forall \alpha \in \mathbb{R}^{*}$, $P_{\alpha}(X)$ and $Q_{\alpha}(X)$ have no root in common, (iii) $\forall \alpha \in \mathbb{R}^{*}$, neither $i$ nor $-i$ is a root of $Q_{\alpha}(X)$, and (iv) $\forall\left(\rho_{0}, \rho_{1}\right) \in \mathbb{R}^{2}$, neither $i$ nor $-i$ is a root of $X^{2}-\rho_{0} X-\rho_{1}$ unless $\left(\rho_{0}, \rho_{1}\right)=(0,-1)$. Therefore, a necessary and sufficient condition for $\mathcal{D}(X)$ to have at most $m=1$ unstable root is $\left(\rho_{0}, \rho_{1}\right) \neq(0,-1)$. As a consequence, Leitemo's (2003) model meets Proposition 7's implementability condition for all admissible values of its structural parameters, except the set of values of measure zero characterized by $\left(\rho_{0}, \rho_{1}\right)=(0,-1)$. Proposition 7 then implies that all feasible paths, in particular the constant-interestrate forecast targeting path, are implementable in this model, for all admissible values of its structural parameters except possibly a set of values of measure zero, when $\mathcal{C B}$ observes past actions and current and past shocks. Moreover, Leitemo's (2003) concept of constant-interest-rate forecast targeting path can easily be extended to cases in which $\mathcal{C B}$ observes actions and shocks with a lag (and in particular does not observe current shocks), as well as to cases in which it observes actions and expectations (but no shocks), again possibly with a lag. Since $\operatorname{rank}\left[\mathbf{A}_{2}(X)\right]=M_{2}$ and $\operatorname{rank}[\mathbf{D}(X)]=M_{1}$ over $\mathbb{R}[X]$ whatever $\left(\delta_{\eta}(X), \delta_{\epsilon}(X)\right) \in \mathbb{R}[X]^{2}$, Propositions 7 and 9 imply that all feasible paths, in particular the constantinterest-rate forecast targeting path, are implementable in these cases too, for all admissible values of the model's structural parameters except possibly again the set of values of measure zero characterized by $\left(\rho_{0}, \rho_{1}\right)=(0,-1)$.

To sum up, for all admissible values of the model's structural parameters (except possibly a set of values of measure zero), a central bank that observes actions and shocks, or actions and expectations,

\footnotetext{
${ }^{53}$ This relationship is a generalization of the relationships between the welfare-relevant output gap $x_{t}$ and the output level $y_{t}$ that were obtained in Section 2 and Subsection 5.1 in the context of micro-founded models.
} 
possibly with lags, can conduct monetary policy on the basis of constant-interest-rate forecasts without generating local equilibrium indeterminacy. This result implies that Leitemo's (2003) point is not against monetary policy based on constant-interest-rate forecasts per se, but only against one specific way of implementing this policy. Basing monetary policy on constant-interest-rate forecasts may well have a number of inherent drawbacks, as argued by, e.g., Leitemo (2003) and Woodford (2007). But, at least in the context of this model and those observation sets, generating local equilibrium indeterminacy is not one of them.

\subsection{Debt-Stabilizing Tax Policy}

Schmitt-Grohé and Uribe (1997) consider, in the standard neoclassical growth model, a labor-income tax-rate rule that balances the government's budget (in the absence of policy implementation lags) or equalizes the expected future value of the stock of public debt to its initial value (in the presence of policy implementation lags). ${ }^{54}$ They find that this rule leads to local equilibrium indeterminacy in both cases for many admissible and empirically relevant values of the structural parameters, whether the labor-income tax is accompanied or not by a capital-income tax that varies in the same proportion. They conclude by warning against the use of labor-income taxes to balance the budget or stabilize the expected future stock of public debt. In this subsection, I apply the general results of the previous section to their framework in order to investigate the robustness of their results and conclusion.

Consider, for instance, Schmitt-Grohé and Uribe's (1997) model in the case where the labor-income tax is accompanied by a capital-income tax that varies in the same proportion, so that it amounts to an income tax. ${ }^{55}$ In this model, at each date $t, \mathcal{P S}$ sets the output level $y_{t}$, the capital stock $k_{t}$, hours worked $h_{t}$, the consumption level $c_{t}$, the (after-tax) rental price of capital $u_{t}$, and the (after-tax) wage $w_{t}$, according to the following structural equations:

$$
\begin{aligned}
y_{t} & =a_{t}+\left(1-s_{h}\right) k_{t}+s_{h} h_{t}, \\
k_{t} & =(1-\delta) k_{t-1}+\frac{\delta}{s_{i}} y_{t-1}-\frac{\delta s_{c}}{s_{i}} c_{t-1}-\frac{\delta\left(1-s_{c}-s_{i}\right)}{s_{i}} g_{t-1}, \\
h_{t} & =\frac{w_{t}}{\gamma}-\frac{c_{t}}{\gamma}, \\
c_{t} & =\mathbb{E}_{t}\left\{c_{t+1}-\chi u_{t+1}\right\} \\
u_{t} & =a_{t}-s_{h}\left(k_{t}-h_{t}\right)-\frac{\tau}{1-\tau} \tau_{t-l}, \\
w_{t} & =a_{t}+\left(1-s_{h}\right)\left(k_{t}-h_{t}\right)-\frac{\tau}{1-\tau} \tau_{t-l},
\end{aligned}
$$

where $\chi \equiv \frac{\delta\left(1-s_{h}\right)(1-\tau)}{\delta\left(1-s_{h}\right)(1-\tau)+(1-\delta) s_{i}} ; \gamma, \delta, s_{c}, s_{h}, s_{i}$, and $\tau$ are such that $\gamma>0,0<\delta<1,0<s_{c}<1$, $0<s_{i}<1,0<s_{c}+s_{i}<1,0<s_{h}<1$, and $0<\tau<1 ; \tau_{t}$ denotes the income tax rate set by the tax authority $\mathcal{T} \mathcal{A}$ at date $t$; and $l \in \mathbb{N}$ denotes the length of the tax-policy-implementation lags. The exogenous productivity and government-purchase disturbances $a_{t}$ and $g_{t}$ follow the $\operatorname{AR}(1)$ processes characterized by

$$
\begin{aligned}
& a_{t}=\theta^{a} a_{t-1}+\epsilon_{t}^{a}, \\
& g_{t}=\theta^{g} g_{t-1}+\epsilon_{t}^{g},
\end{aligned}
$$

\footnotetext{
${ }^{54}$ Most of Schmitt-Grohé and Uribe's (1997) analysis is conducted in continuous time. I refer here to the discrete-time analysis conducted at the end of Section III, in Section IV, and in the Appendix of their paper.

${ }^{55}$ This model amounts to the one detailed in the Appendix of their paper, augmented with technology and governmentpurchase disturbances and policy implementation lags. They use this model without disturbances at the end of Section III, and without policy implementation lags in Section IV.
} 
where $\theta^{a}$ and $\theta^{g}$ are such that $0 \leq \theta^{a}<1$ and $0 \leq \theta^{g}<1$, and $\epsilon_{t}^{a}$ and $\epsilon_{t}^{g}$ are i.i.d. exogenous shocks such that $\mathbb{E}\left\{\epsilon_{t}^{a} \epsilon_{t-k}^{g}\right\}=0$ for any $k \in \mathbb{Z}$. Schmitt-Grohé and Uribe (1997) consider, when $l=0$, an implicit income-tax-rate rule that balances the budget (both in and out of equilibrium) and, when $l \geq 1$, an implicit income-tax-rate rule that stabilizes the expected future stock of public debt (again, both in and out of equilibrium). ${ }^{56}$ They find that both rules lead to local equilibrium indeterminacy for many admissible and empirically relevant values of the structural parameters.

However, the fact that these income-tax-rate rule fail to ensure local equilibrium determinacy does not imply that all the income-tax-rate rules that balance the budget or stabilize the expected future stock of public debt in equilibrium fail to ensure local equilibrium determinacy. So the question remains open as to whether the feasible paths along which the government's budget is balanced or the expected future stock of public debt is stabilized are implementable in this model for empirically plausible $\mathcal{T} \mathcal{A}$ 's observation sets. To answer this question, consider the system made of equations (34) to (41) in the absence of policy implementation lags, i.e. when $l=0$, and rewrite this system in a form of type (6) and (7) with $M_{1}=M_{2}=2, N^{a}=6, N^{e}=0, \boldsymbol{\xi}_{t}=\left[\begin{array}{ll}a_{t} & g_{t}\end{array}\right]^{\prime}, \boldsymbol{\varepsilon}_{t}=\left[\begin{array}{ll}\epsilon_{t}^{a} & \epsilon_{t}^{g}\end{array}\right]^{\prime}, \mathbf{Y}_{t}^{a}=\left[\begin{array}{llllll}y_{t} & k_{t} & h_{t} & c_{t} & u_{t} & w_{t}\end{array}\right]^{\prime}$,

$$
\begin{aligned}
& \mathbf{A}_{1}(L)=\left[\begin{array}{cc}
1-\theta^{a} L & 0 \\
0 & 1-\theta^{g} L
\end{array}\right], \quad \mathbf{A}_{2}(L)=\left[\begin{array}{ll}
1 & 0 \\
0 & 1
\end{array}\right], \quad \mathbf{C}(L)=\left[\begin{array}{llllll}
0 & 0 & 0 & 0 & \frac{\tau}{1-\tau} & \frac{\tau}{1-\tau}
\end{array}\right]^{\prime},
\end{aligned}
$$

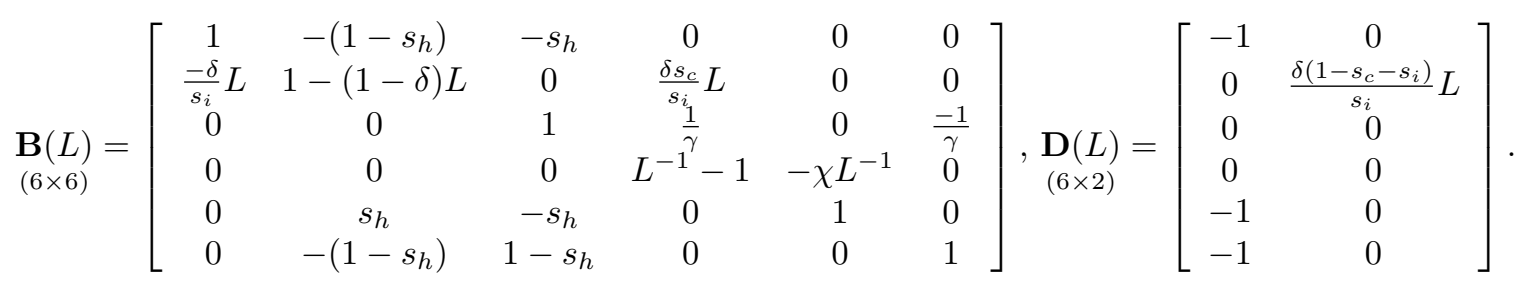

It is easy to check that $\mathbf{B}(L)$ and $\mathbf{C}(L)$ satisfy Assumptions 1 to 4 for all admissible values of the structural parameters $\gamma, \delta, s_{c}, s_{h}, s_{i}$, and $\tau$. Moreover, it is also easy to check that (i) $\Delta_{2}(X)$ is of degree one, which implies that $\mathcal{D}(X)$ has at most $m=1$ unstable root, and (ii) $\operatorname{rank}\left[\mathbf{A}_{2}(X)\right]=M_{2}$ and $\operatorname{rank}[\mathbf{D}(X)]=M_{1}$ over $\mathbb{R}[X]$. Therefore, Schmitt-Grohé and Uribe's (1997) model without policy implementation lags meets Propositions 7 and 9's implementability conditions for all admissible values of its structural parameters. As a consequence, all feasible paths, in particular all feasible paths along which the budget is balanced or the expected future stock of public debt is stabilized, are implementable in this model, for all admissible values of its structural parameters, when $\mathcal{T A}$ observes past actions and shocks, or past actions and expectations, possibly with lags. ${ }^{57}$ Moreover, since implementation lags can be equivalently rewritten as observation lags (as already mentioned in Footnote 33), the statement of the previous sentence is also valid in the presence of policy implementation lags, i.e. when $l \geq 1$.

So, to sum up, for all admissible values of the model's structural parameters, whether in the absence or in the presence of policy implementation lags, a tax authority that observes actions and shocks, or actions and expectations, possibly with lags, can conduct an income-tax policy that balances the budget or stabilizes the expected future stock of public debt in equilibrium without generating local equilibrium

\footnotetext{
${ }^{56}$ The first rule involves $y_{t}$ and $g_{t}$, and the second one $y_{t}, u_{t}, b_{t-1},\left(\mathbb{E}_{t}\left\{y_{t+k}\right\}\right)_{1 \leq k \leq l},\left(\mathbb{E}_{t}\left\{u_{t+k}\right\}\right)_{1 \leq k \leq l},\left(\mathbb{E}_{t}\left\{\tau_{t+k}\right\}\right)_{1 \leq k \leq l}$, and $g_{t}$ (where $b_{t-1}$ denotes the stock of public debt at date $t-1$ ).

${ }^{57}$ It seems natural to assume that $\mathcal{T} \mathcal{A}$ directly observes government-purchase shocks, but not productivity shocks. Therefore, one choice of $\mathcal{T} \mathcal{A}$ 's observation set that seems reasonable is, for instance, $O_{t}^{\mathcal{T} \mathcal{A}}=\left\{\mathbf{Y}^{a, t-1}, \tau^{t-1}, \epsilon^{g, t-1}\right\}$. This observation set is not of a kind considered in Subsection 4.2, since it does not include $\epsilon^{a, t-1}$. However, using the production function (34) and the $\operatorname{AR}(1)$ process (40), $\mathcal{T A}$ can infer past productivity shocks $\epsilon^{a, t-1}$ from its observation of $y^{t-1}, k^{t-1}$, and $h^{t-1}$. Therefore, the set of implementable paths is the same whether $O_{t}^{\mathcal{T} \mathcal{A}}=\left\{\mathbf{Y}^{a, t-1}, \tau^{t-1}, \epsilon^{g, t-1}\right\}$ or $O_{t}^{\mathcal{T} \mathcal{A}}=\left\{\mathbf{Y}^{a, t-1}, \tau^{t-1}, \epsilon^{a, t-1}, \epsilon^{g, t-1}\right\}$. Since the latter observation set is of a kind considered in Subsection 4.2, this set of implementable paths is characterized by Proposition 7's implementability condition.
} 
indeterminacy. This result implies that Schmitt-Grohé and Uribe's (1997) point is not against debtstabilizing labor-income-tax policy per se, but only against one specific way of implementing this policy - one that achieves the policy's goal not only in, but also out of equilibrium. Debt-stabilizing incometax policy may have the inherent drawback of amplifying business cycles, as recalled by Schmitt-Grohé and Uribe (1997). But, at least in the context of their model and those observation sets, and provided that it is not required to achieve its goal also out of equilibrium, this policy does not have the inherent drawback of generating local equilibrium indeterminacy.

\subsection{Optimal Tax Policy}

Correia, Farhi, Nicolini, and Teles (2013) have recently shown, in the context of various models, that an adequate mix of consumption, labor-income, capital-income, profit, and lump-sum taxes can support the first-best allocation even when the (short-term nominal) interest rate is at its zero lower bound. However, they do not address the issue of the local implementation of this optimal tax policy, i.e. the issue of how to set these tax instruments in order to implement the first-best allocation as the unique local equilibrium in the neighborhood of the efficient steady state. Nor do they address the issue of the optimal tax policy and its implementability when the first-best allocation is not feasible, whether because this allocation is not consistent with the observation set of the tax authority $(\mathcal{T A})$, or because policy implementation lags make the tax instruments not flexible enough. Addressing these issues is not trivial, since setting all the tax instruments at their optimal values as functions of only exogenous shocks would lead to local equilibrium indeterminacy in the models that they consider. In this subsection, I address these issues by applying, when necessary, the general results of the previous section to their framework.

Take, for instance, the first and simplest model that they consider, which is Section 2's basic New Keynesian model with (i) constant returns to scale $(\alpha=1)$, (ii) a single kind of labor $\left(\mathbb{1}_{W}=0\right.$ ), (iii) no $u_{t}$ disturbances, (iv) $\nu_{t}=\nu_{1, t}+\nu_{2, t}$, where $\nu_{1, t}$ is a government-purchase disturbance and $\nu_{2, t}$ a preference disturbance, (v) productivity disturbances $a_{t}$, (vi) a consumption tax and a labor-income tax. Assume for simplicity that the interest rate is permanently at its zero lower bound. Assume further that the preference disturbance $\nu_{2, t}$, whose nature is not specified in Correia, Farhi, Nicolini, and Teles (2013), comes from shifts in the consumption-utility function. Next, allow for tax-policy-implementation lags of length $l \in \mathbb{N}$, as in Schmitt-Grohé and Uribe (1997) and the previous subsection. Finally, note $\tau_{t}^{c}$ and $\tau_{t}^{n}$ the consumption-tax and labor-income-tax rates (in levels, not in log-deviations from some steady state), and rewrite equivalently the two policy instruments as $I_{1, t} \equiv \frac{1+\tau_{t}^{c}}{1+\tau_{t-1}^{c}}$ and $I_{2, t} \equiv \frac{1+\tau_{t}^{c}}{1-\tau_{t}^{n}}$ in levels, and as $i_{1, t}$ and $i_{2, t}$ in log-deviations from the efficient steady state. ${ }^{58}$ Then, in the resulting model, at each date $t, \mathcal{P S}$ sets hours worked $n_{t}$, the output level $y_{t}$, the consumption level $c_{t}$, the (pre-tax)

\footnotetext{
${ }^{58}$ I make this change of variables because, when $\mathcal{T} \mathcal{A}$ does not observe any current (exogenous or endogenous) variable, the first-best allocation is feasible only if the log-linearized tax rates follow random walks, whereas it is feasible with stationary processes for $i_{1, t}$ and $i_{2, t}$.
} 
inflation rate $\pi_{t}$, and the (pre-tax) real wage $w_{t}$, according to the following structural equations:

$$
\begin{aligned}
n_{t} & =y_{t}-a_{t} \\
y_{t} & =(1-s) c_{t}+\nu_{1, t} \\
c_{t} & =\mathbb{E}_{t}\left\{c_{t+1}\right\}+\gamma \mathbb{E}_{t}\left\{\pi_{t+1}\right\}+\gamma \mathbb{E}_{t}\left\{i_{1, t+1-l}\right\}-\mathbb{E}_{t}\left\{\nu_{2, t+1}\right\}+\nu_{2, t} \\
\pi_{t} & =\beta \mathbb{E}_{t}\left\{\pi_{t+1}\right\}+\psi\left(w_{t}-a_{t}\right) \\
w_{t} & =\frac{1}{\gamma} c_{t}+\chi n_{t}+i_{2, t-l}-\frac{1}{\gamma} \nu_{2, t}
\end{aligned}
$$

where $\psi \equiv \frac{(1-\theta)(1-\beta \theta)}{\theta}$, and $\beta, \gamma, \chi, s$, and $\theta$ are such that $0<\beta<1, \gamma>0, \chi>0,0<s<1$, and $0<\theta<1 .^{59} \mathrm{I}$ assume that the disturbances $a_{t}, \nu_{1, t}$, and $\nu_{2, t}$, whose stochastic process is not specified in Correia, Farhi, Nicolini, and Teles (2013), follow a VARMA process characterized by

$$
\mathbf{O}(L)\left[\begin{array}{lll}
a_{t} & \nu_{1, t} & \nu_{2, t}
\end{array}\right]^{\prime}=\mathbf{P}(L)\left[\begin{array}{ccc}
\epsilon_{t}^{a} & \epsilon_{t}^{\nu_{1}} & \epsilon_{t}^{\nu_{2}}
\end{array}\right]^{\prime},
$$

where $\mathbf{O}(X)$ and $\mathbf{P}(X)$ are $3 \times 3$ matrices whose elements belong to $\mathbb{R}[X]$, and $\epsilon_{t}^{a}, \epsilon_{t}^{\nu_{1}}$, and $\epsilon_{t}^{\nu_{2}}$ are i.i.d. exogenous shocks such that $\mathbb{E}\left\{\epsilon_{t}^{a} \epsilon_{t-k}^{\nu_{1}}\right\}=\mathbb{E}\left\{\epsilon_{t}^{a} \epsilon_{t-k}^{\nu_{2}}\right\}=\mathbb{E}\left\{\epsilon_{t}^{\nu_{1}} \epsilon_{t-k}^{\nu_{2}}\right\}=0$ for any $k \in \mathbb{Z}$. I also assume, for simplicity and without any loss in generality, that $\mathbf{O}(0)=\mathbf{I}_{3}$.

As shown by Correia, Farhi, Nicolini, and Teles (2013), the first-best allocation is characterized by the production function (42), the binding resource constraint (43), the efficiency condition $\frac{1}{\gamma} c_{t}+\chi n_{t}=$ $a_{t}+\frac{1}{\gamma} \nu_{2, t}$, and the condition that output and consumption levels should be identical across differentiated goods. To be met in a decentralized equilibrium, the latter condition requires $\pi_{t}=0$, and therefore $w_{t}=a_{t}$. It is then easy to check that, when $\mathcal{T} \mathcal{A}$ does not observe any current (exogenous or endogenous) variable, this allocation is feasible if and only if there are no policy implementation lags $(l=0)$ and the policy instruments take the following values in equilibrium:

$$
\begin{gathered}
i_{1, t}=\frac{1+\chi}{1+(1-s) \gamma \chi} a_{t-1}-\frac{\chi}{1+(1-s) \gamma \chi} \nu_{1, t-1}-\frac{(1-s) \chi}{1+(1-s) \gamma \chi} \nu_{2, t-1} \\
\text { and } i_{2, t}=0 .
\end{gathered}
$$

Now, it seems reasonable to assume, for instance, that $\mathcal{T} \mathcal{A}$ directly observes, at date $t, \mathcal{P} \mathcal{S}$ 's actions $n_{t-1}$, $y_{t-1}, c_{t-1}, \pi_{t-1}$, and $w_{t-1}$, its own actions $i_{1, t-1}$ and $i_{2, t-1}$, and the government-purchase disturbance $\nu_{1, t-1}$, but not the productivity disturbance $a_{t-1}$, nor the preference disturbance $\nu_{2, t-1}$. One pair of policy-instrument rules that is consistent with the first-best allocation and such an observation set is the pair made of (49) and the rule that is obtained by (i) using the production function (42) and the first-order condition (46), taken at date $t-1$, to express $a_{t-1}$ and $\nu_{2, t-1}$ as functions of $n_{t-1}, y_{t-1}$, $c_{t-1}, w_{t-1}$, and $i_{2, t-1}$, and (ii) replacing $a_{t-1}$ and $\nu_{2, t-1}$ in (48) by these expressions. However, this pair of rules necessarily leads to local equilibrium indeterminacy, since (i) it has the same local-equilibriumdeterminacy properties as the pair made of (48) and (49), (ii) the system made of the structural equations (42) to (46) and the rules (48) and (49) has the same structure (except for exogenous terms) as the system of equilibrium conditions in Section 2's basic New Keynesian model when the central bank sets the interest rate as a function of only exogenous shocks, and (iii) as is well known, the latter model has the Sargent-Wallace property discussed in Subsection 4.2 for all admissible values of its structural parameters. Now, in this simple framework, local equilibrium determinacy can be easily achieved by

\footnotetext{
${ }^{59}$ For consistency reasons, I use Table 1's notations for these structural parameters, rather than the notations of Correia, Farhi, Nicolini, and Teles (2013).
} 
adding a term $\varphi \pi_{t-1}$ with $\varphi>1$ to the $i_{1, t}$ rule to get

$i_{1, t}=\varphi \pi_{t-1}+\frac{1+\chi}{1+(1-s) \gamma \chi}\left(y_{t-1}-n_{t-1}\right)-\frac{\chi}{1+(1-s) \gamma \chi} \nu_{1, t-1}-\frac{(1-s) \chi}{1+(1-s) \gamma \chi}\left(c_{t-1}+\gamma \chi n_{t-1}-\gamma w_{t-1}\right)$.

Indeed, the system made of the structural equations (42) to (46) and the rules (49) and (50) has the same structure (except for exogenous terms) as the system of equilibrium conditions in Section 2's basic New Keynesian model when the central bank follows the implicit interest-rate rule $i_{t}=\varphi \pi_{t}$, and the latter rule is well known for ensuring local equilibrium determinacy for all admissible values of the structural parameters of this model (as shown by, e.g., Woodford, 2003a, Chapter 4). Moreover, given that $\pi_{t-1}=0$ is consistent with the first-best allocation, this allocation is one, and therefore the unique, local equilibrium under rules (49) and (50). Therefore, the first-best allocation is implementable in this model when there are no policy implementation lags $(l=0)$ and $\mathcal{T} \mathcal{A}$ observes, at date $t, \mathcal{P} \mathcal{S}$ 's actions $n_{t-1}, y_{t-1}, c_{t-1}, \pi_{t-1}$, and $w_{t-1}$, its own actions $i_{1, t-1}$ and $i_{2, t-1}$, and the government-purchase disturbance $\nu_{1, t-1} \cdot{ }^{60}$

The issue of the implementability of optimal tax policy becomes more complex when optimal tax policy cannot support the first-best allocation, i.e. when this allocation is not feasible, for instance because of observation or implementation lags. In such cases, however, it can be easily addressed by applying the general results of the previous section to this specific framework, as I now do. Since these results have been obtained under the assumption of a single policy instrument, let me first write, without any loss in generality, the dynamics of $i_{2, t}$ on the feasible path considered in the VARMA form

$$
Q(L) i_{2, t}=\mathbf{R}(L)\left[\begin{array}{lll}
\epsilon_{t}^{a} & \epsilon_{t}^{\nu_{1}} & \epsilon_{t}^{\nu_{2}}
\end{array}\right]^{\prime}
$$

where $Q(X) \in \mathbb{R}[X]$ and $\mathbf{R}(X)$ is a $1 \times 3$ matrix whose elements belong to $\mathbb{R}[X] .{ }^{61}$ Now consider the model without policy implementation lags $(l=0)$, and rewrite the system made of equations (42) to (47) and (51) in a form of type (6) and (7) with $M_{1}=5, M_{2}=3, N^{a}=5, N^{e}=0, \boldsymbol{\xi}_{t}=$ $\left[\begin{array}{lllll}a_{t} & \nu_{1, t} & \nu_{2, t} & \mathbb{E}_{t}\left\{\nu_{2, t+1}\right\} & i_{2, t}\end{array}\right]^{\prime}, \varepsilon_{t}=\left[\begin{array}{llll}\epsilon_{t}^{a} & \epsilon_{t}^{\nu_{1}} & \epsilon_{t}^{\nu_{2}}\end{array}\right]^{\prime}, \mathbf{Y}_{t}^{a}=\left[\begin{array}{lllll}n_{t} & y_{t} & c_{t} & \pi_{t} & w_{t}\end{array}\right]^{\prime}$,

$$
\begin{aligned}
& \underset{(5 \times 5)}{\mathbf{A}}(L)=\left[\begin{array}{cc|cc}
\mathbf{O}(L) & 0 & 0 \\
& 0 & 0 \\
\hdashline \frac{\mathbf{J}-\mathbf{J O}(L)}{L} & 1 & 0 \\
\hline 0 & 0 & 0 & Q(L)
\end{array}\right], \underset{(5 \times 3)}{\mathbf{A}_{2}(L)}=\left[\begin{array}{c}
\mathbf{P}(L) \\
\frac{\mathbf{J}[\mathbf{P}(L)-\mathbf{P}(0)]}{L} \\
\mathbf{R}(L)
\end{array}\right], \underset{(5 \times 1)}{\mathbf{C}(L)}=\left[\begin{array}{c}
0 \\
0 \\
-\gamma L^{-1} \\
0 \\
0
\end{array}\right], \\
& \underset{(5 \times 5)}{\mathbf{B}(L)}=\left[\begin{array}{ccccc}
1 & -1 & 0 & 0 & 0 \\
0 & 1 & -(1-s) & 0 & 0 \\
0 & 0 & 1-L^{-1} & -\gamma L^{-1} & 0 \\
0 & 0 & 0 & 1-\beta L^{-1} & -\psi \\
-\chi & 0 & \frac{-1}{\gamma} & 0 & 1
\end{array}\right] \text {, and } \underset{(5 \times 5)}{\mathbf{D}(L)}=\left[\begin{array}{ccccc}
1 & 0 & 0 & 0 & 0 \\
0 & -1 & 0 & 0 & 0 \\
0 & 0 & -1 & 1 & 0 \\
\psi & 0 & 0 & 0 & 0 \\
0 & 0 & \frac{1}{\gamma} & 0 & -1
\end{array}\right] \text {, }
\end{aligned}
$$

where $\mathbf{J} \equiv\left[\begin{array}{lll}0 & 0 & 1\end{array}\right]$. It is easy to check that $\mathbf{B}(L)$ and $\mathbf{C}(L)$ satisfy Assumptions 1 to 4 for all admissible values of the structural parameters $\beta, \gamma, \chi, s$, and $\theta$. Moreover, since Section 2's basic New Keynesian model has the Sargent-Wallace property discussed in Subsection 4.2, Proposition 8 implies that $\mathcal{D}(X)$ has at most $m=2$ unstable roots. Therefore, Correia, Farhi, Nicolini, and Teles' (2013) model

\footnotetext{
${ }^{60}$ Of course, it is also implementable when $\mathcal{T} \mathcal{A}$ does not directly observe $\nu_{1, t-1}$ at date $t$, since $\mathcal{T} \mathcal{A}$ can then use the goods-market-clearing condition (43), taken at date $t-1$, to infer $\nu_{1, t-1}$ from its observation of $y_{t-1}$ and $c_{t-1}$.

${ }^{61}$ More generally, in models with several policy instruments, one can (i) set all of them, except one, according to their expressions in the VARMA specification of the feasible path considered, (ii) treat these policy-instrument rules in the same way as structural equations (if they involve some endogenous variables) or as stochastic processes for disturbances (if they do not), and (iii) apply Proposition 7 or 9 to the remaining policy instrument and the feasible path considered.
} 
without policy implementation lags meets Proposition 7's implementability condition for all admissible values of its structural parameters. As a consequence, all feasible paths, in particular the timelessperspective Ramsey-optimal feasible path, are implementable in this model, for all admissible values of its structural parameters, when $\mathcal{T} \mathcal{A}$ observes past actions and shocks, possibly with lags. ${ }^{62}$ Moreover, since implementation lags can be equivalently rewritten as observation lags (as already mentioned in Footnote 33), the statement of the previous sentence is also valid in the presence of policy implementation lags, i.e. when $l \geq 1$.

\section{Conclusion}

This paper addresses the issue of local path implementation in a broad class of locally linearizable dynamic stochastic discrete-time infinite-horizon rational-expectations models, which includes many, if not most, of the models currently used for macroeconomic-stabilization policy analysis. In this class of models, for various alternative policymaker's observation sets, and for any feasible path (i.e. any local path that is consistent with the structural equations and the policymaker's observation set), I provide conditions for this path to be implementable (i.e. for the existence of a policy-instrument rule that is consistent with the policymaker's observation set and implements this path as the unique local equilibrium). Moreover, for any implementable path, I show how to design a corresponding policyinstrument rule arithmetically, and thus obtain its coefficients as rational functions of the structural and implementable-path parameters. Finally, I apply these general results to, and derive their implications for, the implementation of some specific stabilization policies, namely monetary policy, monetary policy based on constant-interest-rate forecasts, debt-stabilizing tax policy, and optimal tax policy, in some specific models.

Overall, the view that should emerge from the paper is that the policymaker's observation set is a key ingredient of the environment that should be explicitly stated alongside other ingredients such as preferences, technology, and markets. Not only does the set of feasible paths depend on this observation set, but, for a given set of feasible paths, so does the (possibly strict) subset of implementable paths. Consider, for instance, the timeless-perspective Ramsey-optimal feasible path, which is often considered as a benchmark in normative analyses. The literature that considers this path typically focuses on observation sets for the policymaker that make her observation constraint slack (e.g., observation sets that include all current and past exogenous shocks, possibly among other variables). In many instances, however, there is an alternative, empirically more relevant observation set for the policymaker, which makes her observation constraint binding (e.g., an observation set that does not include current endogenous or exogenous variables). So, another natural benchmark would be the timeless-perspective Ramsey-optimal feasible path corresponding to this alternative observation set. And still another natural benchmark would be the corresponding timeless-perspective Ramsey-optimal implementable path. I have shown that the first benchmark may provide, in the basic New Keynesian model, a new explanation for the observed smoothness of interest-rate paths chosen by central banks over time, and that the second

\footnotetext{
${ }^{62}$ As already mentioned, one choice of $\mathcal{T} \mathcal{A}$ 's observation set that seems reasonable is, for instance, $O_{t}^{\mathcal{T} \mathcal{A}=}$ $\left\{\mathbf{Y}^{a, t-1}, i_{1}^{t-1}, i_{2}^{t-1}, \epsilon^{\nu_{1}, t-1}\right\}$. This observation set is not of a kind considered in Subsection 4.2, since it does not include $\epsilon^{a, t-1}$ nor $\epsilon^{\nu_{2}, t-1}$. However, using the production function $(42)$ and the first-order condition (46), $\mathcal{T} \mathcal{A}$ can infer $\epsilon^{a, t-1}$ and $\epsilon^{\nu_{2}, t-1}$ from this observation set. Therefore, the set of implementable paths is the same whether $O_{t}^{\mathcal{T} \mathcal{A}}=\left\{\mathbf{Y}^{a, t-1}, i_{1}^{t-1}, i_{2}^{t-1}, \epsilon^{\nu_{1}, t-1}\right\}$ or $O_{t}^{\mathcal{T} \mathcal{A}}=\left\{\mathbf{Y}^{a, t-1}, i_{1}^{t-1}, i_{2}^{t-1}, \epsilon^{a, t-1}, \epsilon^{\nu_{1}, t-1}, \epsilon^{\nu_{2}, t-1}\right\}$. Since the latter observation set is of a kind considered in Subsection 4.2, this set of implementable paths is characterized by Proposition 7's implementability condition.
} 
benchmark may differ from the first one in Svensson and Woodford's (2005) model even when there is one more endogenous variable in the central bank's observation set than exogenous shocks in the model.

The analysis conducted in this paper could be extended in at least two interesting directions. First, I have focused on some specific kinds of observation sets for the policymaker. Other kinds of observation sets could be considered (in particular sets that include, among other variables, some but not all exogenous shocks), and the set of implementable paths could be characterized for each of them. Second, none of the policy-instrument rules that I have designed in this paper is unique. In fact, for each observation set and each implementable path, there exists an infinity of rules that are consistent with this observation set and implement that path as the unique local equilibrium. ${ }^{63}$ This multiplicity opens the door to the design of rules with additional properties. This degree of freedom could be illustrated by designing, for instance, non-superinertial policy-instrument rules. ${ }^{64}$ I leave these two extensions for future research.

\section{Appendix B}

In this appendix, I prove Lemma 1, Proposition 7, and Proposition 9. Before proceeding, let me introduce the following notations. For any system of equations $(S)$, let $\mathbb{E}_{t}\{(S)\}$ (respectively $L(S)$ ) denote the system obtained by applying operator $\mathbb{E}_{t}$ (respectively $L$ ) on both the left- and the right-hand sides of each equation of $(S)$. For any non-zero integers $n$ and $p$, any $n \times p$ matrix $K$ and any $p$-equation system $(S)$, let $K(S)$ denote the $n$-equation system obtained by applying $K$ to both the $p$-element vector made of the left-hand sides of the equations of $(S)$ and the $p$-element vector made of their right-hand sides - so that, in particular, for any $N$-equation system $(S)$ and any $j \in\{1, \ldots, N\}$, $\mathbf{e}_{j}^{\prime}(S)$ denotes the $j^{\text {th }}$ equation of $(S)$. For any non-zero integer $n$, let $\mathbf{I}_{n}$ denote the $n \times n$ identity matrix. For any polynomial $\mathcal{H}(X) \in \mathbb{R}[X]$, let $d_{\mathcal{H}}$ denote the degree of $\mathcal{H}(X)$. Lastly, let $|$.$| denote the modulus operator (when$ applied to a complex number), the determinant operator (when applied to a matrix), or the cardinality operator (when applied to a set).

\section{B.1 Proof of Lemma 1}

Consider a system of structural equations $(S)$ of type $(7)$ and a policy-instrument rule $(R)$ of type (8) satisfying (11). I proceed in two steps: first, I show that the system made of $(S)$ and $(R)$ can be written in Blanchard and Kahn's (1980) form with $m$ non-predetermined variables; second, I show that its non-zero eigenvalues are the non-zero roots of (12).

Step 1: for each $j \in\{1, \ldots, N\}$, if $j \in J_{C}$ then replace sequentially, for $k=0, \ldots, m_{j}^{c}+m, \mathbb{E}_{t}\left\{i_{t+m_{j}^{c}-k}\right\}$ (if it appears) in $\mathbf{e}_{j}^{\prime}(S)$ by its expression in $\mathbb{E}_{t}\left\{L^{k-m_{j}^{c}}(R)\right\}$. Let $(\widetilde{S})$ denote the resulting system, and let $\left(\widetilde{m}_{j}^{b}\right)_{1 \leq j \leq N}$ and $\widehat{\widetilde{\mathbf{B}}}(L)$ denote the counterparts of $\left(m_{j}^{b}\right)_{1 \leq j \leq N}$ and $\widehat{\mathbf{B}}(L)$ for $(\widetilde{S})$. Note that $\widehat{\widetilde{\mathbf{B}}}(0)=\widehat{\mathbf{B}}(0)$, since $(R)$ satisfies $(11)$. The rest of Step 1 is essentially devoted to the rewriting of $(\widetilde{S})$. For simplicity, I will keep the same notations $(\widetilde{S}),\left(\widetilde{m}_{j}^{b}\right)_{1 \leq j \leq N}$ and $\widehat{\widetilde{\mathbf{B}}}(L)$ throughout the rewriting process. The previous

\footnotetext{
${ }^{63}$ These rules may differ, for instance, in terms of the unstable eigenvalues of the implied $(N+1)$-equation system (made of the structural equations and the rule considered).

${ }^{64}$ The term "superinertial" was coined by Woodford (1999b). In my framework, rules of type (8) satisfying (10) are said to be superinertial when the polynomial $X^{n^{g}} G\left(X^{-1}\right)$ has at least one unstable root. In the literature on optimal monetary policy, superinertial interest-rate rules are common. They can be found in, e.g., Giannoni and Woodford (2002, 2003, 2005), Rotemberg and Woodford (1999), and Woodford (1999b, 2003a, Chapter 8). Their pervasiveness has led Woodford (1999b, p. 32) to call for "further investigation of the robustness of the conclusion that the largest autoregressive root should exceed one under an optimal policy" in models of the monetary transmission mechanism. This extension would answer his call.
} 
operation ensures that none of the rewritten systems $(\widetilde{S})$ will include any variable of type $\mathbb{E}_{t}\left\{i_{t+k}\right\}$ for $k \in \mathbb{N}$. Now re-order the lines of $(\widetilde{S})$ so that $\widetilde{m}_{1}^{b} \geq \ldots \geq \widetilde{m}_{N}^{b}$. Let $K \in\{1, \ldots, N\}$ and $\left(j_{1}, \ldots, j_{K}\right) \in$ $\{1, \ldots, N\}^{K}$ be such that $\widetilde{m}_{1}^{b}=\ldots=\widetilde{m}_{j_{1}}^{b}>\widetilde{m}_{j_{1}+1}^{b}=\ldots=\widetilde{m}_{j_{2}}^{b}>\ldots>\widetilde{m}_{j_{K-1}+1}^{b}=\ldots=\widetilde{m}_{j_{K}}^{b}=\widetilde{m}_{N}^{b}$. Re-order the columns of $\widetilde{\mathbf{B}}(L)$ so that $\forall j \in\{1, \ldots, N-1\}$, the $(N-j) \times(N-j)$ matrix noted $\mathbf{M}_{j}$ obtained by removing the first $j$ lines and the first $j$ columns from $\widehat{\widetilde{\mathbf{B}}}(0)$ is invertible, this re-ordering being made possible by Assumption 3. Re-order the elements of $\mathbf{Y}_{t}$ accordingly and note $\widetilde{\mathbf{Y}}_{t}$ the resulting vector. For each $j \in\left\{1, \ldots, j_{1}\right\}$, replace $\mathbf{e}_{j}^{\prime}(\widetilde{S})$ by

$$
\mathbf{e}_{j}^{\prime} \widehat{\widetilde{\mathbf{B}}}(0)^{-1} \mathbb{E}_{t}\left[\begin{array}{cccc}
1 & 0 & \cdots & 0 \\
0 & L^{\widetilde{m}_{2}^{b}-\widetilde{m}_{1}^{b}} & \ddots & \vdots \\
\vdots & \ddots & \ddots & 0 \\
0 & \cdots & 0 & L^{\widetilde{m}_{N}^{b}-\widetilde{m}_{1}^{b}}
\end{array}\right](\widetilde{S})
$$

This operation makes the first $j_{1}$ lines of $\widehat{\widetilde{\mathbf{B}}}(0)$ identical to the first $j_{1}$ lines of the $N \times N$ identity matrix. If $K=1$, then the system made of $(\widetilde{S})$ and $(R)$, which is equivalent to the system made of $(S)$ and $(R)$, can easily be written in Blanchard and Kahn's (1980) form with $j_{1} \widetilde{m}_{j_{1}}^{b}=\sum_{j=1}^{N} m_{j}^{b}=m$ non-predetermined variables. Alternatively, if $K \geq 2$, then for each $i \in\left\{j_{1}+1, \ldots, N\right\}$ and each $j \in\left\{1, \ldots, j_{1}\right\}$, replace sequentially, for $k=1, \ldots, \widetilde{m}_{j_{1}}^{b}-\widetilde{m}_{j_{2}}^{b}, \mathbb{E}_{t}\left\{\mathbf{e}_{i}^{\prime} \widetilde{\mathbf{Y}}_{t+\widetilde{m}_{j_{1}}^{b}-k}\right\}$ (if it appears) in $\mathbf{e}_{j}^{\prime}(\widetilde{S})$ by its expression in

$$
\mathbf{M}_{j_{1}}^{-1} \mathbb{E}_{t}\left\{\left[\begin{array}{cccc|cccc}
0 & 0 & \cdots & 0 & L^{\widetilde{m}_{j_{1}+1}^{b}-\widetilde{m}_{j_{1}}^{b}+k} & 0 & \cdots & 0 \\
0 & \ddots & \ddots & \vdots & 0 & \ddots & \ddots & \vdots \\
\vdots & \ddots & \ddots & 0 & \vdots & \ddots & \ddots & 0 \\
0 & \cdots & 0 & 0 & 0 & \cdots & 0 & L^{\widetilde{m}_{N}^{b}-\widetilde{m}_{j_{1}}^{b}+k}
\end{array}\right](\widetilde{S})\right\}
$$

This operation does not affect the first $j_{1}$ lines of $\widehat{\widetilde{\mathbf{B}}}(0)$, and ensures in particular that for each $i \in$ $\left\{j_{1}+1, \ldots, j_{2}\right\}$, (i) $(\widetilde{S})$ does not include any variable of type $\mathbb{E}_{t}\left\{\mathbf{e}_{i}^{\prime} \widetilde{\mathbf{Y}}_{t+k}\right\}$ with $\widetilde{m}_{j_{2}}^{b}<k<\widetilde{m}_{j_{1}}^{b}$, and (ii) $\mathbf{e}_{j_{1}+1}^{\prime}(\widetilde{S}), \ldots, \mathbf{e}_{j_{2}}^{\prime}(\widetilde{S})$ are the only lines of $(\widetilde{S})$ in which $\mathbb{E}_{t}\left\{\mathbf{e}_{i}^{\prime} \widetilde{\mathbf{Y}}_{t+\widetilde{m}_{j_{2}}^{b}}\right\}$ may appear. Proceed in a similar way as previously to transform $\mathbf{e}_{j}^{\prime}(\widetilde{S})$ for $j \in\left\{j_{1}+1, \ldots, j_{2}\right\}$, then (if $K \geq 3$ ) $\mathbf{e}_{j}^{\prime}(\widetilde{S})$ for $j \in\left\{j_{2}+1, \ldots, j_{3}\right\}$, and so on up to $\mathbf{e}_{j}^{\prime}(\widetilde{S})$ for $j \in\left\{j_{K-1}+1, \ldots, j_{K}\right\}$. Then, for each $i \in\{1, \ldots, N\}$, (i) the only variable of type $\mathbb{E}_{t}\left\{\mathbf{e}_{i}^{\prime} \widetilde{\mathbf{Y}}_{t+k}\right\}$ with $k \geq \min \left\{\widetilde{m}_{j}^{b} \mid 1 \leq j \leq N\right\}$ that appears in $(\widetilde{S})$ is $\mathbb{E}_{t}\left\{\mathbf{e}_{i}^{\prime} \widetilde{\mathbf{Y}}_{t+\widetilde{m}_{i}^{b}}\right\}$, and (ii) this variable appears only in line $\mathbf{e}_{i}^{\prime}(\widetilde{S})$, and its coefficient is one (as $\widehat{\widetilde{\mathbf{B}}}(0)$ is the $N \times N$ identity matrix). Therefore, the system made of $(\widetilde{S})$ and $(R)$, which is equivalent to the system made of $(S)$ and $(R)$, can then easily be written in Blanchard and Kahn's (1980) form with $\sum_{k=1}^{K} j_{k} \widetilde{m}_{j_{k}}^{b}=\sum_{j=1}^{N} m_{j}^{b}=m$ non-predetermined variables.

Step 2: let $I_{C} \equiv\left\{j \in J_{C} \mid m_{j}^{c} \geq m_{j}^{b}\right\}$. For each $j \in I_{C}, \exists K_{j}(X) \in \mathbb{R}[X]$ such that $L^{m_{j}^{b}} \mathbf{e}_{j}^{\prime} \mathbf{C}(L)$ $L^{m_{j}^{b}-m_{j}^{c}} K_{j}(L) G(L)$ is of the form $\sum_{k=1}^{n^{\Phi_{j}}} \Phi_{j, k} L^{k}$ where $n^{\Phi_{j}} \in \mathbb{N}^{*}$ and all $\Phi_{j, k}$ are real numbers. Now, the non-zero eigenvalues of the system made of $(S)$ and $(R)$ are those of the corresponding perfect-foresight deterministic system

$$
\left[\begin{array}{l|l}
\mathbf{B}(L) & \mathbf{C}(L) \\
\hline \mathbf{F}(L) & G(L)
\end{array}\right]\left[\begin{array}{c}
\mathbf{Y}_{t} \\
i_{t}
\end{array}\right]=0
$$

which are in turn those of $\mathbf{\Psi}_{1}(L)\left[\begin{array}{cc}\mathbf{Y}_{t}^{\prime} & i_{t}\end{array}\right]^{\prime}=0$ with

$$
\mathbf{\Psi}_{1}(L) \equiv\left[\begin{array}{c|c} 
& L^{m_{1}^{b}} \mathbf{e}_{1}^{\prime} \mathbf{C}(L) \\
\widehat{\mathbf{B}}(L) & \vdots \\
& L^{m_{N}^{b}} \mathbf{e}_{N}^{\prime} \mathbf{C}(L) \\
\hline \mathbf{F}(L) & G(L)
\end{array}\right]
$$


or equivalently those of $\mathbf{\Psi}_{2}(L)\left[\begin{array}{cc}\mathbf{Y}_{t}^{\prime} & i_{t}\end{array}\right]^{\prime}=0$ with $\underset{((N+1) \times(N+1))}{\mathbf{\Psi}_{2}(L)} \equiv \sum_{k=0}^{n^{\psi_{2}}} \mathbf{\Psi}_{2, k} L^{k}=$

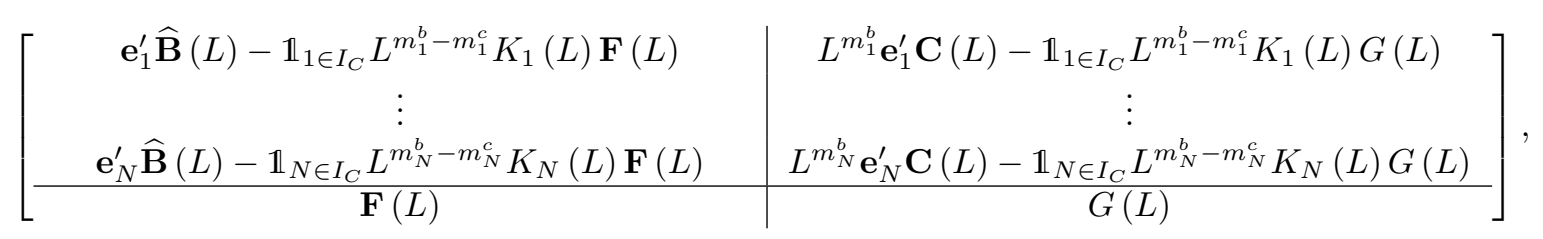

where $n^{\psi_{2}} \in \mathbb{N}$ and all $\boldsymbol{\Psi}_{2, k}$ have real numbers as elements. Since $(R)$ satisfies (11),

$$
\boldsymbol{\Psi}_{2,0}=\left[\begin{array}{ccc|c} 
& & & 0 \\
& \widehat{\mathbf{B}}(0) & & \vdots \\
& & & 0 \\
\hline 0 & \cdots & 0 & g_{0}
\end{array}\right] .
$$

Since $|\widehat{\mathbf{B}}(0)| \neq 0$ and $g_{0} \neq 0, \Psi_{2,0}$ is invertible, so that according to a standard result of time-series analysis (see, e.g., Hamilton, 1994, Chapter 10, Proposition 10.1) the non-zero eigenvalues of the system made of $(S)$ and $(R)$ are the non-zero roots of polynomial $\left|X^{n^{\psi_{2}}} \boldsymbol{\Psi}_{2}\left(X^{-1}\right)\right| \in \mathbb{R}[X]$. Note that $\boldsymbol{\Psi}_{2}(L)$ has been obtained by adding $-L^{m_{j}^{b}-m_{j}^{c}} K_{j}(L) \mathbf{v}_{N+1}^{\prime} \boldsymbol{\Psi}_{1}(L)$ to $\mathbf{v}_{j}^{\prime} \boldsymbol{\Psi}_{1}(L)$ for each $j \in I_{C}$. Now, adding a scalar multiple of one row to another row leaves the determinant of a matrix unchanged. Therefore, $\left|X^{n^{\psi_{2}}} \boldsymbol{\Psi}_{2}\left(X^{-1}\right)\right|=\left|X^{n^{\psi_{2}}} \boldsymbol{\Psi}_{1}\left(X^{-1}\right)\right|$. Finally, $\left|X^{n^{\psi_{2}}} \boldsymbol{\Psi}_{1}\left(X^{-1}\right)\right|$ is equal to (12) up to a multiplicative factor of type $X^{z}$ with $z \in \mathbb{Z}$. Therefore, the non-zero roots of $\left|X^{n^{\psi_{2}}} \boldsymbol{\Psi}_{1}\left(X^{-1}\right)\right|$ are those of (12). Lemma 1 follows.

\section{B.2 Proof of Proposition 7}

Assume that $\mathcal{D}(X)$ has at most $m$ unstable roots (taking into account their multiplicity). Consider a given path of type (9) satisfying (16), (17), and (18), and note it $(P)$. I proceed in two steps: first, I design some $\mathbf{F}(L)$ satisfying (13) and (14) and some $G(L)$ such that, whatever $\mathbf{H}(L)$, the system made of the structural equations (7) and the corresponding policy-instrument rule (8) satisfying (10) has at most one stationary solution; second, I design $\mathbf{H}(L)$ satisfying (15) such that $(P)$ is one, and hence the unique, stationary solution of this system.

Step 1: Bézout's identity implies that there exists $\left(\mathcal{U}_{i}(X)\right)_{i \in\left\{1, \ldots, N^{a}\right\} \cup\{N+1\}} \in \mathbb{R}[X]^{N^{a}+1}$ such that

$$
\sum_{i \in\left\{1, \ldots, N^{a}\right\} \cup\{N+1\}} \mathcal{U}_{i}(X) \Delta_{i}(X)=\mathcal{D}(X) .
$$

Let $\Theta(X) \equiv\left|X^{n^{s}} \mathbf{S}\left(X^{-1}\right)\right| \in \mathbb{R}[X]$. Let $\mathcal{Z}(X)$ be a given polynomial such that (i) $\mathcal{Z}(X) \mathcal{D}(X)$ has exactly $m$ unstable roots (taking into account their multiplicity), and (ii) $\Theta(X)$ is a divisor of $\mathcal{Z}(X) \mathcal{D}(X)$. The existence of $\mathcal{Z}(X)$ is a consequence of the assumptions that (i) $\mathcal{D}(X)$ has at most $m$ unstable roots (taking into account their multiplicity), and (ii) all the roots of $\Theta(X)$ are stable. Let $n \in \mathbb{N}$ be such that

$$
n \geq 2 d_{\Delta_{N+1}}-d_{\mathcal{D}}-d_{\mathcal{Z}}+\max \left\{d_{\mathcal{U}_{N+1}}, \max _{i \in\left\{1, \ldots, N^{a}\right\}}\left(d_{\mathcal{U}_{i}}\right)+\max \left\{l_{Y}, \max _{j \in J_{C}}\left(m_{j}^{c}-m_{j}^{b}\right)\right\}\right\} .
$$

Finally, let $\mathcal{Q}(X) \in \mathbb{R}[X]$ and $\mathcal{R}(X) \in \mathbb{R}[X]$ denote respectively the quotient and the remainder of the Euclidean division of $X^{n} \mathcal{Z}(X)$ by $\Delta_{N+1}(X)$, i.e. the unique polynomials such that $X^{n} \mathcal{Z}(X)=$ $\Delta_{N+1}(X) \mathcal{Q}(X)+\mathcal{R}(X)$ with $d_{\mathcal{R}}<d_{\Delta_{N+1}}$. Multiplying the left- and right-hand sides of (B.1) by $\mathcal{R}(X)$, I obtain $\mathcal{R}(X) \sum_{i \in\left\{1, \ldots, N^{a}\right\}} \mathcal{U}_{i}(X) \Delta_{i}(X)=\mathcal{R}(X) \mathcal{D}(X)$ and therefore

$$
\begin{gathered}
\sum_{i \in\left\{1, \ldots, N^{a}\right\}}\left[\mathcal{R}(X) \mathcal{U}_{i}(X)\right] \Delta_{i}(X)+\left[\mathcal{R}(X) \mathcal{U}_{N+1}(X)+\mathcal{Q}(X) \mathcal{D}(X)\right] \Delta_{N+1}(X) \\
=X^{n} \mathcal{Z}(X) \mathcal{D}(X) .
\end{gathered}
$$


Let $\mathcal{F}_{i}(X) \equiv \mathcal{R}(X) \mathcal{U}_{i}(X)$ for $i \in\left\{1, \ldots, N^{a}\right\}, \mathcal{F}_{i}(X) \equiv 0$ for $i \in\left\{N^{a}+1, \ldots, N\right\}$ (if $N^{e} \geq 1$ ), and $\mathcal{G}(X) \equiv \mathcal{R}(X) \mathcal{U}_{N+1}(X)+\mathcal{Q}(X) \mathcal{D}(X)$. Let $A_{1, j}(L)$ denote the $j^{\text {th }}$ element of the diagonal of $\mathbf{A}_{1}(L)$ for each $j \in\left\{1, \ldots, M_{1}\right\}$, and let $A_{1}(L) \equiv \prod_{j=1}^{M_{1}} A_{1, j}(L)$. The choice of $G(L) \equiv A_{1}(L) L^{d_{\mathcal{G}}} \mathcal{G}\left(L^{-1}\right)$ is admissible since it implies that $G(L)$ is of type $\sum_{k=0}^{n^{g}} g_{k} L^{k}$, where $n^{g} \in \mathbb{N}$, all $g_{k}$ are real numbers, and $g_{0} \neq 0$. Moreover, given that

$$
\begin{aligned}
& \left\{\begin{array}{l}
n \geq 2 d_{\Delta_{N+1}}-d_{\mathcal{D}}-d_{\mathcal{Z}}+\max \left\{d_{\mathcal{U}_{N+1},} \max _{i \in\left\{1, \ldots, N^{a}\right\}}\left(d_{\mathcal{U}_{i}}\right)+\max \left\{l_{Y}, \max _{j \in J_{C}}\left(m_{j}^{c}-m_{j}^{b}\right)\right\}\right\} \\
n=d_{\Delta_{N+1}}+d_{\mathcal{Q}}-d_{\mathcal{Z}} \\
d_{\Delta_{N+1}}>d_{\mathcal{R}}
\end{array}\right. \\
& \Longrightarrow d_{\mathcal{Q}}+d_{\mathcal{D}}>d_{\mathcal{R}}+\max \left\{d_{\mathcal{U}_{N+1},} \max _{i \in\left\{1, \ldots, N^{a}\right\}}\left(d_{\mathcal{U}_{i}}\right)+\max \left\{l_{Y}, \max _{j \in J_{C}}\left(m_{j}^{c}-m_{j}^{b}\right)\right\}\right\} \\
& \Longrightarrow\left\{\begin{array}{l}
d_{\mathcal{Q}}+d_{\mathcal{D}}>d_{\mathcal{R}}+d_{\mathcal{U}_{N+1}} \\
d_{\mathcal{Q}}+d_{\mathcal{D}}>d_{\mathcal{R}}+\max _{i \in\left\{1, \ldots, N^{a}\right\}}\left(d_{\mathcal{U}_{i}}\right)+\max \left\{l_{Y}, \max _{j \in J_{C}}\left(m_{j}^{c}-m_{j}^{b}\right)\right\}
\end{array}\right. \\
& \Longrightarrow d_{\mathcal{G}}=d_{\mathcal{Q}}+d_{\mathcal{D}}>d_{\mathcal{F}_{i}}+\max \left\{l_{Y}, \max _{j \in J_{C}}\left(m_{j}^{c}-m_{j}^{b}\right)\right\} \text { for } i \in\left\{1, \ldots, N^{a}\right\},
\end{aligned}
$$

the choice of $\mathbf{F}(L) \equiv A_{1}(L) L^{d_{\mathcal{G}}} \sum_{i=1}^{N}(-1)^{N+1-i} \mathcal{F}_{i}\left(L^{-1}\right) \mathbf{e}_{i}^{\prime}$ is admissible too since it implies that (i) $\mathbf{F}(L)$ is of type $\sum_{k=1}^{n^{f}} \mathbf{F}_{k} L^{k}$, where $n^{f} \in \mathbb{N}^{*}$ and all $\mathbf{F}_{k}$ are $1 \times N$ matrices whose elements are real numbers, and (ii) $\mathbf{F}(L)$ satisfies (13) and (14). Finally, this choice is such that $\mathbf{F}(L)$ also satisfies (11). Lemma 1 then implies that any rule of type (8) with $\mathbf{E}_{1}(L)=\mathbf{0}$ and these $\mathbf{F}(L)$ and $G(L)$ is such that (i) the system made of the structural equations (7) and this rule can be written in Blanchard and Kahn's (1980) form with $m$ non-predetermined variables, and (ii) the non-zero eigenvalues of this system are the non-zero roots of (12) and hence, given (B.2), the non-zero roots of $X^{d_{A_{1}}} A_{1}\left(X^{-1}\right) \mathcal{Z}(X) \mathcal{D}(X)$. Now, $X^{d_{A_{1}}} A_{1}\left(X^{-1}\right) \mathcal{Z}(X) \mathcal{D}(X)$ has exactly $m$ unstable roots (taking into account their multiplicity). As a consequence, the system made of the structural equations (7) and any policy-instrument rule (8) with $\mathbf{E}_{1}(L)=\mathbf{0}$ and these $\mathbf{F}(L)$ and $G(L)$ meets Blanchard and Kahn's (1980) order condition and has either one or zero stationary solution, depending on whether it meets Blanchard and Kahn's (1980) rank condition.

Step 2: consider first the case in which $\max \left\{m_{j}^{c}-m_{j}^{b} \mid j \in J_{C}\right\} \leq 0$. Along the path $(P)$, there exist (i) a unique $1 \times M_{2}$ matrix $\boldsymbol{\Xi}(L) \equiv \sum_{k=l_{\varepsilon}}^{+\infty} \boldsymbol{\Xi}_{k} L^{k}$, where all $\boldsymbol{\Xi}_{k}$ have real numbers as elements, such that

$$
\mathbf{F}(L) \mathbf{Y}_{t}+G(L) i_{t}+\boldsymbol{\Xi}(L) \varepsilon_{t}=\mathbf{0}
$$

where $\mathbf{F}(L)$ and $G(L)$ are the ones designed in Step 1, and (ii) a unique $N \times M_{2}$ matrix $\Pi(L) \equiv$ $\left[\begin{array}{lll}\sum_{k=0}^{m_{1}^{b}-1} \Pi_{1, k} L^{k} & \cdots & \sum_{k=0}^{m_{N}^{b}-1} \boldsymbol{\Pi}_{N, k} L^{k}\end{array}\right]^{\prime}$, where all $\boldsymbol{\Pi}_{j, k}$ have real numbers as elements, such that

$$
\left[\begin{array}{c|c} 
& L^{m_{1}^{b}} \mathbf{e}_{1}^{\prime} \mathbf{C}(L) \\
\widehat{\mathbf{B}}(L) & \vdots \\
& L^{m_{N}^{b}} \mathbf{e}_{N}^{\prime} \mathbf{C}(L)
\end{array}\right]\left[\begin{array}{c}
\mathbf{Y}_{t} \\
i_{t}
\end{array}\right]+\left[\begin{array}{c}
L^{m_{1}^{b}} \mathbf{e}_{1}^{\prime} \mathbf{D}(L) \\
\vdots \\
L^{m_{N}^{b}} \mathbf{e}_{N}^{\prime} \mathbf{D}(L)
\end{array}\right] \boldsymbol{\xi}_{t}+\boldsymbol{\Pi}(L) \boldsymbol{\varepsilon}_{t}=\mathbf{0} .
$$


Multiplying the left- and right-hand sides of (B.4) by $A_{1}(L)$ leads to

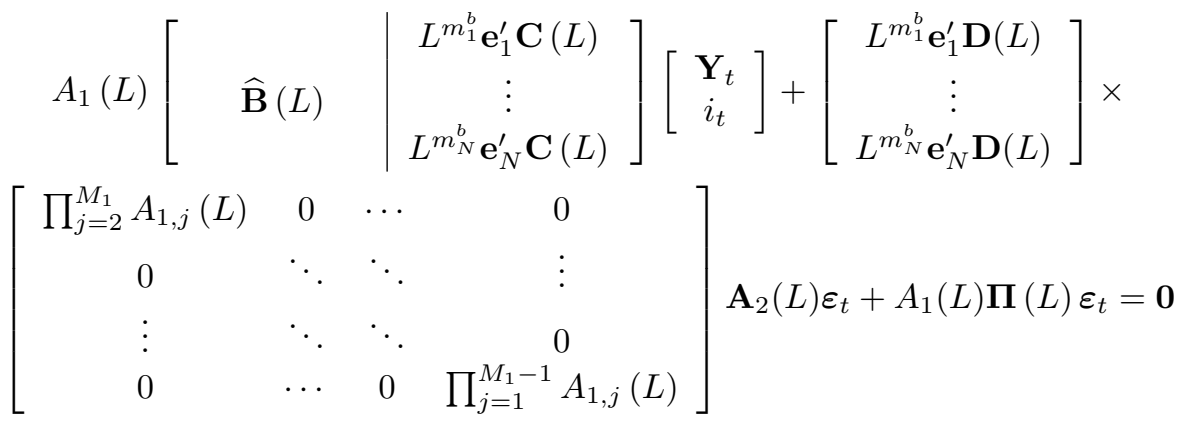

since, as a scalar, $A_{1}(L)$ is such that $A_{1}(L) \mathbf{K}=\mathbf{K} A_{1}(L)$ for any matrix $\mathbf{K}$. The system made of (B.3) and (B.5) is of VARMA type $\boldsymbol{\Lambda}_{1}(L)\left[\begin{array}{ll}\mathbf{Y}_{t}^{\prime} & i_{t}\end{array}\right]^{\prime}=\boldsymbol{\Lambda}_{2}(L) \boldsymbol{\varepsilon}_{t}$ with $\left|\boldsymbol{\Lambda}_{1}(0)\right| \neq 0$, since $\left|\mathbf{A}_{1}(0)\right| \neq 0$, $|\widehat{\mathbf{B}}(0)| \neq 0, \max \left\{m_{j}^{c}-m_{j}^{b} \mid j \in J_{C}\right\} \leq 0, \mathbf{F}(0)=\mathbf{0}$, and $G(0) \neq 0$. Cramer's rule then implies that there exist $\left(n_{1}, \ldots, n_{N+1}\right) \in \mathbb{N}^{N+1}$, where $n_{j} \geq d_{\Delta_{j}}$ for $j \in\{1, \ldots, N+1\}$, and an $(N+1) \times M_{2}$ matrix $\boldsymbol{\Gamma}(L) \equiv \sum_{k=0}^{n^{\gamma}} \boldsymbol{\Gamma}_{k} L^{k}$, where $n^{\gamma} \in \mathbb{N}$ and all $\boldsymbol{\Gamma}_{k}$ have real numbers as elements, such that this system can be rewritten

$$
\begin{aligned}
& A_{1}(L) L^{d_{\mathcal{Z}}+d_{\mathcal{D}}} \mathcal{Z}\left(L^{-1}\right) \mathcal{D}\left(L^{-1}\right)\left[\begin{array}{ll}
\mathbf{Y}_{t}^{\prime} & i_{t}
\end{array}\right]^{\prime} \\
& =\boldsymbol{\Gamma}(L) \varepsilon_{t}+\left[\begin{array}{lll}
L^{n_{1}} \Delta_{1}\left(L^{-1}\right) & \cdots & L^{n_{N+1}} \Delta_{N+1}\left(L^{-1}\right)
\end{array}\right]^{\prime} \boldsymbol{\Xi}(L) \varepsilon_{t},
\end{aligned}
$$

given Step 1. But Cramer's rule also implies that there exists an $(N+1) \times M_{2}$ matrix $\mathbf{\Upsilon}(L) \equiv \sum_{k=0}^{n^{v}} \Upsilon_{k} L^{k}$, where $n^{v} \in \mathbb{N}$ and all $\Upsilon_{k}$ have real numbers as elements, such that the path $(P)$ can be rewritten $\left.L^{d_{\Theta} \Theta\left(L^{-1}\right)\left[\mathbf{Y}_{t}^{\prime} \quad i_{t}\right.}\right]^{\prime}=\mathbf{\Upsilon}(L) \varepsilon_{t}$, which implies

$$
\begin{aligned}
& A_{1}(L) L^{d_{\mathcal{Z}}+d_{\mathcal{D}}} \mathcal{Z}\left(L^{-1}\right) \mathcal{D}\left(L^{-1}\right)\left[\begin{array}{ll}
\mathbf{Y}_{t}^{\prime} & i_{t}
\end{array}\right]^{\prime} \\
& =A_{1}(L) \frac{L^{d_{\mathcal{Z}}+d_{\mathcal{D}}} \mathcal{Z}\left(L^{-1}\right) \mathcal{D}\left(L^{-1}\right)}{\left.L^{d_{\Theta} \Theta\left(L^{-1}\right.}\right)} \Upsilon(L) \varepsilon_{t}
\end{aligned}
$$

where $\frac{X^{d} \mathcal{Z}+d_{\mathcal{D}} \mathcal{Z}\left(X^{-1}\right) \mathcal{D}\left(X^{-1}\right)}{X^{d} \Theta \Theta\left(X^{-1}\right)}$ is of finite degree by construction of $\mathcal{Z}(X)$. Given that Assumption 3 implies $\Delta_{N+1}(X) \neq 0$, the identification of (B.6) with (B.7) shows that $\boldsymbol{\Xi}(X)$ is of finite degree too. Thus, the choice of $\mathbf{H}(L) \equiv \boldsymbol{\Xi}(L)$ is admissible, since it implies that (i) $\mathbf{H}(L)$ is of type $\sum_{k=0}^{n^{h}} \mathbf{H}_{k} L^{k}$, where $n^{h} \in \mathbb{N}$ and all $\mathbf{H}_{k}$ are $1 \times M_{2}$ matrices whose elements are real numbers, and (ii) $\mathbf{H}(L)$ satisfies (15). In the alternative case in which $\max \left\{m_{j}^{c}-m_{j}^{b} \mid j \in J_{C}\right\}>0$, an admissible $\mathbf{H}(L)$ can be designed in a similar way by using the fact that the $\mathbf{F}(L)$ designed in Step 1 satisfies (11). Therefore, the rule of type (8) with $\mathbf{E}_{1}(L)=\mathbf{0}$ and the $\mathbf{F}(L), G(L)$, and $\mathbf{H}(L)$ designed in Steps 1 and 2 satisfies (10), (13), (14), and (15), and is such that (i) the system made of (7) and this rule has at most one stationary solution, and (ii) the path $(P)$ is one stationary solution of that system. Proposition 7 follows.

\section{B.3 Proof of Proposition 9}

Assume that $\mathcal{D}(X)$ has at most $m$ unstable roots (taking into account their multiplicity). Consider a given $l_{Y} \in \mathbb{N}$ and a given path of type (9) satisfying (22) and (23). This path can be rewritten in an equivalent form of type (9) satisfying (16), (17), and (18) with $l_{\varepsilon}=l_{Y}+1$. Then, Proposition 7 applied to this path implies that there exists an arithmetically designable policy-instrument rule of type

$$
\widetilde{\mathbf{F}}(L) \mathbf{Y}_{t}+\widetilde{G}(L) i_{t}+\widetilde{\mathbf{H}}(L) \varepsilon_{t}=0
$$




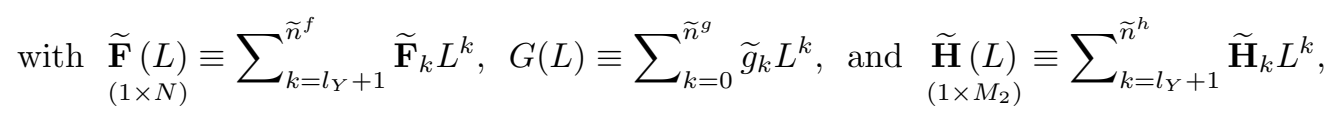

where $\left(\widetilde{n}^{f}, \widetilde{n}^{h}\right) \in\left[\mathbb{N} \backslash\left\{0, \ldots, l_{Y}\right\}\right]^{2}, \widetilde{n}^{g} \in \mathbb{N}$, all $\widetilde{g}_{k}$ are real numbers, $\widetilde{g}_{0} \neq 0$, and all $\widetilde{\mathbf{F}}_{k}, \widetilde{\mathbf{H}}_{k}$ have real numbers as elements, such that this path is the unique stationary solution of the system made of the structural equations (7) and that rule (B.8). Assume moreover that $\operatorname{rank}\left[\mathbf{A}_{2}(X)\right]=M_{2}$ over $\mathbb{R}[X]$, i.e. that $\forall\left(P_{1}(X), \ldots, P_{M_{2}}(X)\right) \in \mathbb{R}[X]^{M_{2}} \backslash(0, \ldots, 0), \mathbf{A}_{2}(X)\left[\begin{array}{lll}P_{1}(X) & \ldots & P_{M_{2}}(X)\end{array}\right]^{\prime} \neq \mathbf{0}$. Then, (6) implies that there exist

$$
\underset{\left(M_{2} \times M_{1}\right)}{\widetilde{\mathbf{A}}_{1}(L)} \equiv \sum_{k=-\widetilde{m}^{a_{1}}}^{\widetilde{\mathbf{n}}^{a_{1}}} \widetilde{\mathbf{A}}_{1, k} L^{k} \text { and } \underset{\left(M_{2} \times M_{2}\right)}{\widetilde{\mathbf{A}}_{2}(L)} \equiv\left[\begin{array}{cccc}
\widetilde{A}_{2,1}(L) & 0 & \ldots & 0 \\
0 & \ddots & \ddots & \vdots \\
\vdots & \ddots & \ddots & 0 \\
0 & \ldots & 0 & \widetilde{A}_{2, M_{2}}(L)
\end{array}\right] \text {, }
$$

where $\widetilde{A}_{2, j}(L) \equiv \sum_{k=0}^{\widetilde{n}^{a_{2}}} \widetilde{a}_{2, j, k} L^{k}$ for $1 \leq j \leq M_{2},\left(\widetilde{m}^{a_{1}}, \widetilde{n}^{a_{1}}, \widetilde{n}^{a_{2}}\right) \in \mathbb{N}^{3}$, all $\widetilde{\mathbf{A}}_{1, k}$ have real numbers as elements, all $\widetilde{a}_{2, j, k}$ are real numbers, and $\widetilde{\mathbf{A}}_{2}(0)=\mathbf{I}_{M_{2}}$, such that $\widetilde{\mathbf{A}}_{2}(L) \boldsymbol{\varepsilon}_{t}=\widetilde{\mathbf{A}}_{1}(L) \boldsymbol{\xi}_{t}$. Assume finally that $\operatorname{rank}[\mathbf{D}(X)]=M_{1}$ over $\mathbb{R}[X]$, i.e. that $\forall\left(P_{1}(X), \ldots, P_{M_{1}}(X)\right) \in \mathbb{R}[X]^{M_{1}} \backslash(0, \ldots, 0)$, $\mathbf{D}(X)\left[\begin{array}{lll}P_{1}(X) & \ldots & P_{M_{1}}(X)\end{array}\right]^{\prime} \neq \mathbf{0}$. Then, (7) implies that there exist

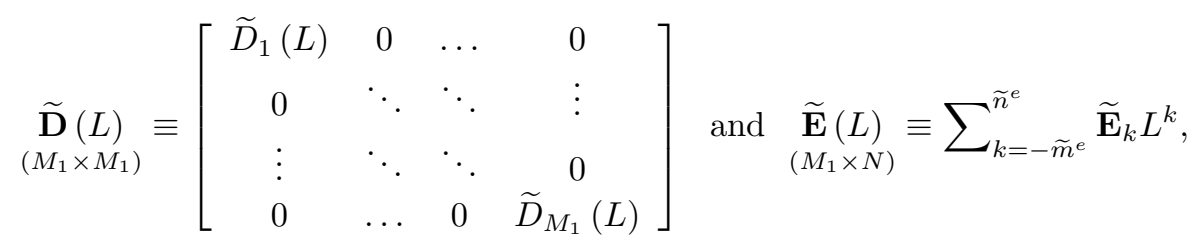

where $\widetilde{D}_{j}(L) \equiv \sum_{k=0}^{\widetilde{n}^{d}} \widetilde{d}_{j, k} L^{k}$ for $1 \leq j \leq M_{1},\left(\widetilde{m}^{e}, \widetilde{n}^{d}, \widetilde{n}^{e}\right) \in \mathbb{N}^{3}$, all $\widetilde{\mathbf{E}}_{k}$ have real numbers as elements, all $\widetilde{d}_{j, k}$ are real numbers, and $\widetilde{\mathbf{D}}(0)=\mathbf{I}_{M_{1}}$, such that $\widetilde{\mathbf{D}}(L) \boldsymbol{\xi}_{t}=\widetilde{\mathbf{E}}(L) \mathbb{E}_{t}\left\{\mathbf{B}(L) \mathbf{Y}_{t}+\mathbf{C}(L) i_{t}\right\}$. Multiplying the left- and right-hand sides of (B.8) by $P(L) \equiv \prod_{j=1}^{M_{1}} \widetilde{D}_{j}(L) \prod_{j=1}^{M_{2}} \widetilde{A}_{2, j}(L)$ leads to the rule

$$
\begin{aligned}
& P(L) \widetilde{\mathbf{F}}(L) \mathbf{Y}_{t}+P(L) \widetilde{G}(L) i_{t}+\widetilde{\mathbf{H}}(L)\left[\begin{array}{cccc}
\prod_{j=2}^{M_{2}} \widetilde{A}_{2, j}(L) & 0 & \ldots & 0 \\
0 & \ddots & \ddots & \vdots \\
\vdots & \ddots & \ddots & 0 \\
0 & \ldots & 0 & \prod_{j=1}^{M_{2}-1} \widetilde{A}_{2, j}(L)
\end{array}\right] \widetilde{\mathbf{A}}_{1}(L) \\
& {\left[\begin{array}{cccc}
\prod_{j=2}^{M_{1}} \widetilde{D}_{j}(L) & 0 & \ldots & 0 \\
0 & \ddots & \ddots & \vdots \\
\vdots & \ddots & \ddots & 0 \\
0 & \ldots & 0 & \prod_{j=1}^{M_{1}-1} \widetilde{D}_{j}(L)
\end{array}\right] \widetilde{\mathbf{E}}(L) \mathbb{E}_{t}\left\{\mathbf{B}(L) \mathbf{Y}_{t}+\mathbf{C}(L) i_{t}\right\}=0,}
\end{aligned}
$$

since $P(L) \varepsilon_{t}=\mathbb{E}_{t}\left\{P(L) \varepsilon_{t}\right\}$. The rule (B.9) is of type (8) and satisfies (19), (20), and (21). Moreover, the transformation of (B.8) into (B.9) is neutral from the local-equilibrium-determinacy point of view. Proposition 9 follows.

\section{References}

[1] Atkeson, A., Chari, V.V., and Kehoe, P.J. (2010), "Sophisticated Monetary Policies," Quarterly Journal of Economics, 125(1), 47-89.

[2] d'Autume, A. (1990), "On the Solution of Linear Difference Equations with Rational Expectations," Review of Economic Studies, 57(4), 677-688. 
[3] Bassetto, M. (2002), "A Game-Theoretic View of the Fiscal Theory of the Price Level," Econometrica, 70(6), 2167-2195.

[4] Bassetto, M. (2004), "Negative Nominal Interest Rates," American Economic Review, 94(2), 104108.

[5] Bassetto, M. (2005), "Equilibrium and Government Commitment," Journal of Economic Theory, 124(1), 79-105.

[6] Benhabib, J. (2004), "Interest Rate Policy in Continuous Time with Discrete Delays," Journal of Money, Credit and Banking, 36(1), 1-15.

[7] Benhabib, J., Schmitt-Grohé, S., and Uribe, M. (2002), "Avoiding Liquidity Traps," Journal of Political Economy, 110(3), 535-563.

[8] Blanchard, O.J., and Kahn, C.M. (1980), "The Solution of Linear Difference Models under Rational Expectations," Econometrica, 48(5), 1305-1311.

[9] Bullard, J., and Mitra, K. (2002), "Learning About Monetary Policy Rules," Journal of Monetary Economics, 49(6), 1105-1129.

[10] Carlstrom, C.T., and Fuerst, T.S. (2002), "Taylor Rules in a Model that Satisfies the Natural-Rate Hypothesis," American Economic Review, Papers and Proceedings, 92(2), 79-84.

[11] Christiano, L.J., Eichenbaum, M., and Evans, C.L. (2005), "Nominal Rigidities and the Dynamic Effects of a Shock to Monetary Policy," Journal of Political Economy, 113(1), 1-45.

[12] Christiano, L.J., Motto, R., and Rostagno, M. (2013), "Risk Shocks," forthcoming in American Economic Review.

[13] Christiano, L.J., and Rostagno, M. (2001), "Money Growth Monitoring and the Taylor Rule," NBER Working Paper No. 8539.

[14] Clarida, R., Galí, J., and Gertler, M. (1999), "The Science of Monetary Policy: A New Keynesian Perspective," Journal of Economic Literature, 37(4), 1661-1707.

[15] Clarida, R., Galí, J., and Gertler, M. (2000), "Monetary Policy Rules and Macroeconomic Stability: Evidence and Some Theory," Quarterly Journal of Economics, 115(1), 147-180.

[16] Cochrane, J.H. (2011), "Determinacy and Identification with Taylor Rules," Journal of Political Economy, 119(3), 565-615.

[17] Correia, I., Farhi, E., Nicolini, J.P., and Teles, P. (2013), "Unconventional Fiscal Policy at the Zero Bound," American Economic Review, 103(4), 1172-1211.

[18] Eggertsson, G.B., and Swanson, E.T. (2008), "Optimal Time-Consistent Monetary Policy in the New Keynesian Model with Repeated Simultaneous Play," mimeo, Federal Reserve Bank of New York and Federal Reserve Bank of San Francisco.

[19] Evans, G.W., and Honkapohja, S. (2006), "Monetary Policy, Expectations and Commitment," Scandinavian Journal of Economics, 108(1), 15-38.

[20] Galí, J. (2008), Monetary Policy, Inflation and the Business Cycle: An Introduction to the New Keynesian Framework, Princeton: Princeton University Press.

[21] Galí, J. (2011), “Are Central Banks' Projections Meaningful?," Journal of Monetary Economics, $58(6-8), 537-550$.

[22] Giannoni, M.P., and Woodford, M. (2002), "Optimal Interest-Rate Rules: I. General Theory," NBER Working Paper No. 9419.

[23] Giannoni, M.P., and Woodford, M. (2003), "How Forward-Looking Is Optimal Monetary Policy?," Journal of Money, Credit and Banking, 35(6), 1425-1469. 
[24] Giannoni, M.P., and Woodford, M. (2005), "Optimal Inflation-Targeting Rules," in Bernanke, B.S., and Woodford, M. (eds) The Inflation-Targeting Debate, Chicago: NBER and University of Chicago Press, 93-162.

[25] Giannoni, M.P., and Woodford, M. (2010), "Optimal Target Criteria for Stabilization Policy," NBER Working Paper No. 15757.

[26] Hamilton, J.D. (1994), Time Series Analysis, Princeton: Princeton University Press.

[27] King, R.G., and Wolman, A.L. (2004), "Monetary Discretion, Pricing Complementarity, and Dynamic Multiple Equilibria," Quarterly Journal of Economics, 119(4), 1513-1553.

[28] Klein, P. (2000), "Using the Generalized Schur Form to Solve a Multivariate Linear Rational Expectations Model," Journal of Economic Dynamics and Control, 24(10), 1405-1423.

[29] Leeper, E.M., Sims, C.A., and Zha, T. (1996), "What Does Monetary Policy Do?," Brooking Papers on Economic Activity, No. 2, 1-78.

[30] Leitemo, K. (2003), "Targeting Inflation by Constant-Interest-Rate Forecasts," Journal of Money, Credit and Banking, 35(4), 609-626.

[31] Loisel, O. (2009), "Bubble-Free Policy Feedback Rules," Journal of Economic Theory, 144(4), 15211559.

[32] McCallum, B.T. (1981), "Price Level Determinacy with an Interest Rate Policy Rule and Rational Expectations," Journal of Monetary Economics, 8(3), 319-329.

[33] McCallum, B.T. (1999), "Issues in the Design of Monetary Policy Rules," in Taylor, J.B., and Woodford, M. (eds) Handbook of Macroeconomics, Amsterdam: Elsevier Science, 1C, 1483-1530.

[34] Mertens, K., and Ravn, M. (2012), "Empirical Evidence on the Aggregate Effects of Anticipated and Unanticipated U.S. Tax Policy Shocks," American Economic Journal: Economic Policy, 4(2), 145-181.

[35] Orphanides, A. (2001), "Monetary Policy Rules Based on Real-Time Data," American Economic Review, 91(4), 964-985.

[36] Poole, W. (1970), "Optimal Choice of Monetary Policy Instruments in a Simple Stochastic Macro Model," Quarterly Journal of Economics, 84(2), 197-216.

[37] Prasolov, V.V. (2004), Polynomials, in Bronstein, M., Cohen, A.M., Cohen, H., Eisenbud, D., and Sturmfels, B. (eds) Algorithms and Computation in Mathematics, Berlin: Springer-Verlag, 11.

[38] Rotemberg, J.J., and Woodford, M. (1999), "Interest Rate Rules in an Estimated Sticky Price Model," in Taylor, J.B. (ed) Monetary Policy Rules, Chicago: NBER and University of Chicago Press, 57-119.

[39] Sargent, T.J., and Wallace, N. (1975), "Rational Expectations, the Optimal Monetary Intrument and the Optimal Money Supply Rule," Journal of Political Economy, 83(2), 241-254.

[40] Schmitt-Grohé, S., and Uribe, M. (1997), "Balanced-Budget Rules, Distortionary Taxes, and Aggregate Instability," Journal of Political Economy, 105(5), 976-1000.

[41] Sims, C.A. (2002), "Solving Linear Rational Expectations Models," Computational Economics, 20(1-2), 1-20.

[42] Smets, F., and Wouters, R. (2007), "Shocks and Frictions in US Business Cycles: A Bayesian DSGE Approach," American Economic Review, 97(3), 586-606.

[43] Svensson, L.E.O. (1997), "Inflation Forecast Targeting: Implementing and Monitoring Inflation Targets," European Economic Review, 41(6), 1111-1146. 
[44] Svensson, L.E.O. (1999), "Inflation Targeting as a Monetary Policy Rule," Journal of Monetary Economics, 43(3), 607-654.

[45] Svensson, L.E.O. (2003), "What is Wrong with Taylor Rules? Using Judgment in Monetary Policy through Targeting Rules," Journal of Economic Literature, 41(2), 426-477.

[46] Svensson, L.E.O. (2011), "Inflation targeting," in Friedman, B.M., and Woodford, M. (eds) Handbook of Monetary Economics, Amsterdam: Elsevier Science, 3B, 1237-1302.

[47] Svensson, L.E.O., and Woodford, M. (2005), "Implementing Optimal Policy Through InflationForecast Targeting," in Bernanke, B.S., and Woodford, M. (eds) The Inflation-Targeting Debate, Chicago: NBER and University of Chicago Press, 19-83.

[48] Taylor, J.B. (1993), "Discretion Versus Policy Rules in Practice," Carnegie-Rochester Conference Series on Public Policy, 39, 195-214.

[49] Woodford, M. (1999a), "Commentary: How Should Monetary Policy Be Conducted in an Era of Price Stability?," in New Challenges for Monetary Policy, Kansas City: Federal Reserve Bank of Kansas City, 277-316.

[50] Woodford, M. (1999b), "Optimal Monetary Policy Inertia," The Manchester School 67(S), 1-35.

[51] Woodford, M. (2000), "Pitfalls of Forward-Looking Monetary Policy," American Economic Review, Papers and Proceedings, 90(2), 100-104.

[52] Woodford, M. (2003a), Interest and Prices: Foundations of a Theory of Monetary Policy, Princeton: Princeton University Press.

[53] Woodford, M. (2003b), "Optimal Interest-Rate Smoothing," Review of Economic Studies, 70(4), 861-886.

[54] Woodford, M. (2007), "Forecast Targeting as a Monetary Policy Strategy: Policy Rules in Practice," NBER Working Paper No. 13716. 


\section{Appendix A (Not For Publication)}

In this appendix, I prove Propositions 2, 3, 4, 6, and 11.

\section{A.1 Proof of Proposition 2}

Consider an interest-rate rule of type

$$
i_{t}=a \pi_{t-1}+b y_{t-1}+c \nu_{t}+d u_{t}+e \nu_{t-1}+f u_{t-1},
$$

where $(a, b, c, d, e, f) \in \mathbb{R}^{6}$. I proceed in three steps: first, I derive a sufficient condition on $(a, b, c, d, e, f)$ for the system made of (1), (2) and (A.1) to have a unique stationary solution $\left(\pi_{t}, y_{t}, i_{t}\right)_{t \in \mathbb{Z}}$; second, I derive a necessary and sufficient condition on $(a, b, c, d, e, f)$ for the path (4) to be one stationary solution of this system; third, I show that these two conditions are equivalent to the conditions mentioned in Proposition 2.

Step 1: the system made of (1), (2) and (A.1) is equivalent to the system made of

$$
\mathbb{E}_{t}\left\{\left[\begin{array}{c}
\pi_{t+1} \\
\pi_{t} \\
y_{t+1} \\
y_{t}
\end{array}\right]\right\}=\left[\begin{array}{cccc}
\frac{1}{\beta} & 0 & \frac{-\kappa}{\beta} & 0 \\
1 & 0 & 0 & 0 \\
\frac{-\sigma}{\beta} & \sigma a & 1+\frac{\kappa \sigma}{\beta} & \sigma b \\
0 & 0 & 1 & 0
\end{array}\right]\left[\begin{array}{c}
\pi_{t} \\
\pi_{t-1} \\
y_{t} \\
y_{t-1}
\end{array}\right]+\left[\begin{array}{c}
\frac{\kappa \phi}{\beta} \nu_{t}-\frac{1}{\beta} u_{t} \\
0 \\
\Phi_{t} \\
0
\end{array}\right],
$$

where $\Phi_{t} \equiv\left(\sigma c-\frac{\kappa \phi \sigma}{\beta}-1\right) \nu_{t}+\left(\sigma d+\frac{\sigma}{\beta}\right) u_{t}+\sigma e \nu_{t-1}+\sigma f u_{t-1}$, which characterizes the dynamics of $\pi$ and $y$, and (A.1), which uniquely determines the dynamics of $i$ from those of $\pi$ and $y$. Therefore, this system has a unique stationary solution $\left(\pi_{t}, y_{t}, i_{t}\right)_{t \in \mathbb{Z}}$ if and only if (A.2) has a unique stationary solution $\left(\pi_{t}, y_{t}\right)_{t \in \mathbb{Z}}$. Now, (A.2) is written in Blanchard and Kahn's (1980) form with two non-predetermined variables. Moreover, (A.2) satisfies Blanchard and Kahn's (1980) rank condition, as can be easily checked. As a consequence, (A.2) has a unique stationary solution $\left(\pi_{t}, y_{t}\right)_{t \in \mathbb{Z}}$ if and only if it satisfies Blanchard and Kahn's (1980) order condition, that is to say if and only if it has exactly two unstable eigenvalues, or equivalently if and only if the corresponding characteristic polynomial $X \mathcal{P}(X)$, where $\mathcal{P}(X) \equiv \beta X^{3}+$ $\omega_{2} X^{2}+\omega_{1} X+\omega_{0}$ with $\omega_{2} \equiv-(1+\beta+\kappa \sigma), \omega_{1} \equiv 1-\beta \sigma b$ and $\omega_{0} \equiv \kappa \sigma a+\sigma b$, has exactly two unstable roots (taking into account their multiplicity). Since $\mathcal{P}(X) \rightarrow-\infty$ as $X \rightarrow-\infty$ and $\mathcal{P}(X) \rightarrow+\infty$ as $X \rightarrow+\infty$ for $X \in \mathbb{R}$, one sufficient condition for that is $\mathcal{P}(-1)=-\beta+\omega_{2}-\omega_{1}+\omega_{0}>0$ and $\mathcal{P}(1)=\beta+\omega_{2}+\omega_{1}+\omega_{0}<0$. Given that $\sigma>0$, this condition can be equivalently rewritten

$$
\kappa a+(1+\beta) b>\kappa+\frac{2(1+\beta)}{\sigma} \text { and } \kappa a+(1-\beta) b<\kappa .
$$

Step 2: by construction, (1) and (2) are consistent with (4). Moreover, since $\nu_{t}$ and $u_{t}$ are independent of each other, (A.1) is consistent with the path (4) if and only if $c=\frac{1-\phi}{\sigma}, d=\frac{(\kappa-\lambda \sigma) \mu(1-\mu)}{\lambda \sigma}, e+\phi b=0$, $f+\mu a-\frac{\kappa \mu}{\lambda} b=\frac{(\kappa-\lambda \sigma) \mu^{2}(1-\mu)}{\lambda \sigma}$ and $\frac{\lambda(1-\mu)}{\kappa} a+\mu b=\frac{-(\kappa-\lambda \sigma) \mu^{2}(1-\mu)}{\kappa \sigma}$, i.e. if and only if

$$
b=\frac{-\lambda(1-\mu)}{\kappa \mu} a-\frac{(\kappa-\lambda \sigma) \mu(1-\mu)}{\kappa \sigma}, c=\frac{1-\phi}{\sigma}, d=\frac{(\kappa-\lambda \sigma) \mu(1-\mu)}{\lambda \sigma}, e=-\phi b \text { and } f=-a .
$$

Step 3: from Steps 1 and 2, I get that a sufficient condition for the path (4) to be the unique stationary solution of the system made of (1), (2) and (A.1) is that (A.3) and (A.4) jointly hold. Now, the system 
made of (A.3) and (A.4) is equivalent to the system made of (A.4),

$$
\begin{aligned}
& {\left[\frac{\kappa^{2} \mu-(1+\beta) \lambda(1-\mu)}{\kappa \mu}\right] a>\frac{\kappa^{2} \sigma+(1+\beta)[2 \kappa+(\kappa-\lambda \sigma) \mu(1-\mu)]}{\kappa \sigma}} \\
& \text { and }\left[\frac{\kappa^{2} \mu-(1-\beta) \lambda(1-\mu)}{\kappa \mu}\right] a<\frac{\kappa^{2} \sigma+(1-\beta)(\kappa-\lambda \sigma) \mu(1-\mu)}{\kappa \sigma} .
\end{aligned}
$$

Moreover, using $\beta \lambda \mu^{2}-\left(\lambda+\beta \lambda+\kappa^{2}\right) \mu+\lambda=0$ and $0<\mu<1$, I get

$$
\begin{gathered}
\frac{\kappa^{2} \mu-(1+\beta) \lambda(1-\mu)}{\kappa \mu}=\frac{\left(\lambda+\beta \lambda+\kappa^{2}\right) \mu-\lambda-\beta \lambda}{\kappa \mu}=\frac{-\beta \lambda\left(1-\mu^{2}\right)}{\kappa \mu}<0 \\
\text { and } \frac{\kappa^{2} \mu-(1-\beta) \lambda(1-\mu)}{\kappa \mu}=\frac{\left(\lambda-\beta \lambda+\kappa^{2}\right) \mu-\lambda+\beta \lambda}{\kappa \mu}=\frac{\beta \lambda(1-\mu)^{2}}{\kappa \mu}>0 .
\end{gathered}
$$

Therefore, the system made of (A.3) and (A.4) is equivalent to the system made of (A.4),

$$
a<-\frac{\kappa^{2} \sigma \mu+(1+\beta)\left[2 \kappa \mu+(\kappa-\lambda \sigma) \mu^{2}(1-\mu)\right]}{\beta \lambda \sigma\left(1-\mu^{2}\right)} \text { and } a<\frac{\kappa^{2} \sigma \mu+(1-\beta)(\kappa-\lambda \sigma) \mu^{2}(1-\mu)}{\beta \lambda \sigma(1-\mu)^{2}} .
$$

Proposition 2 follows.

\section{A.2 Proof of Proposition 3}

Given that the timeless-perspective Ramsey-optimal feasible path is defined as the limit of the date$t_{0}$ Ramsey-optimal feasible path as $t_{0} \rightarrow-\infty$, I proceed in four steps: in the first step, I write the optimization problem that defines the date- $t_{0}$ Ramsey-optimal feasible path; in the second and third steps, I solve this problem and determine the date- $t_{0}$ Ramsey-optimal feasible path; in the fourth step, I deduce the timeless-perspective Ramsey-optimal feasible path from the date- $t_{0}$ Ramsey-optimal feasible path.

Step 1: the date- $t_{0}$ Ramsey-optimal feasible path is defined as the state-contingent path for the endogenous variables that maximizes welfare at date $t_{0}$ subject to the structural equations and $\mathcal{C B}$ 's observation constraint. To determine this path, I follow the undetermined-coefficients method and specify the inflation rate and the output level in the following general linear way: $\pi_{t_{0}+k}=\sum_{j=0}^{k} a_{j, k}^{\pi} \nu_{t_{0}+k-j}+$ $\sum_{j=0}^{k} b_{j, k}^{\pi} u_{t_{0}+k-j}$ and $y_{t_{0}+k}=\phi \nu_{t_{0}+k}+\sum_{j=0}^{k} a_{j, k}^{y} \nu_{t_{0}+k-j}+\sum_{j=0}^{k} b_{j, k}^{y} u_{t_{0}+k-j}$ for $k \geq 0$. I seek the values of coefficients $\left(a_{j, k}^{\pi}, b_{j, k}^{\pi}, a_{j, k}^{y}, b_{j, k}^{y}\right)_{k \geq 0,0 \leq j \leq k}$ that minimize

$$
\begin{aligned}
L_{t_{0}}=\mathbb{E}_{t_{0}}\left\{\sum _ { k = 0 } ^ { + \infty } \beta ^ { k } \left[\left(\sum_{j=0}^{k} a_{j, k}^{\pi} \nu_{t_{0}+k-j}+\sum_{j=0}^{k} b_{j, k}^{\pi} u_{t_{0}+k-j}\right)^{2}\right.\right. & \\
& \left.\left.+\lambda\left(\sum_{j=0}^{k} a_{j, k}^{y} \nu_{t_{0}+k-j}+\sum_{j=0}^{k} b_{j, k}^{y} u_{t_{0}+k-j}\right)^{2}\right]\right\}
\end{aligned}
$$

subject to the following constraints:

$$
\begin{aligned}
& \phi+a_{0, k}^{y}-a_{1, k+1}^{y}-\sigma a_{1, k+1}^{\pi}-1=0 \text { for } k \geq 0, \\
& a_{j, k}^{\pi}-\beta a_{j+1, k+1}^{\pi}-\kappa a_{j, k}^{y}=0 \text { for } k \geq 0 \text { and } j \in\{0, \ldots, k\}, \\
& b_{0, k}^{y}-b_{1, k+1}^{y}-\sigma b_{1, k+1}^{\pi}=0 \text { for } k \geq 0, \\
& b_{0, k}^{\pi}-\beta b_{1, k+1}^{\pi}-\kappa b_{0, k}^{y}-1=0 \text { for } k \geq 0, \\
& b_{j, k}^{\pi}-\beta b_{j+1, k+1}^{\pi}-\kappa b_{j, k}^{y}=0 \text { for } k \geq 1 \text { and } j \in\{1, \ldots, k\} .
\end{aligned}
$$


These constraints come from (i) the structural equations (1) and (2), (ii) the assumption that $\mathbb{E}\left\{\nu_{t} u_{t-k}\right\}$ $=0$ for any $k \in \mathbb{Z}$, and (iii) the assumption on $\mathcal{C B}$ 's observation set, which implies that $i_{t}$ cannot depend on $\left(\nu_{t}, u_{t}\right)$. I note respectively $\left(\Omega_{k}^{I S, a}\right)_{k \geq 0},\left(\Omega_{j, k}^{P C, a}\right)_{k \geq 0,0 \leq j \leq k},\left(\Omega_{k}^{I S, b}\right)_{k \geq 0},\left(\Omega_{0, k}^{P C, b}\right)_{k \geq 0}$ and $\left(\Omega_{j, k}^{P C, b}\right)_{k \geq 1,1 \leq j \leq k}$ the Lagrange multipliers associated with these constraints. Since $\mathbb{E}\left\{\nu_{t} u_{t-k}\right\}=0$ for any $k \in \mathbb{Z}$, coefficients $\left(a_{j, k}^{\pi}, a_{j, k}^{y}\right)_{k \geq 0,0 \leq j \leq k}$ are determined separately from coefficients $\left(b_{j, k}^{\pi}, b_{j, k}^{y}\right)_{k \geq 0,0 \leq j \leq k}$.

Step 2: let me first focus on the determination of coefficients $\left(a_{j, k}^{\pi}, a_{j, k}^{y}\right)_{k \geq 0,0 \leq j \leq k}$. The first-order conditions of the Lagrangian's minimization with respect to these coefficients are

$$
\begin{aligned}
2 V_{\nu} \beta^{k} a_{0, k}^{\pi}-\Omega_{0, k}^{P C, a} & =0 \text { for } k \geq 0, \\
2 V_{\nu} \beta^{k} a_{1, k}^{\pi}+\sigma \Omega_{k-1}^{I S, a}-\Omega_{1, k}^{P C, a}+\beta \Omega_{0, k-1}^{P C, a} & =0 \text { for } k \geq 1, \\
2 V_{\nu} \beta^{k} a_{j, k}^{\pi}-\Omega_{j, k}^{P C, a}+\beta \Omega_{j-1, k-1}^{P C, a} & =0 \text { for } k \geq 2 \text { and } j \in\{2, \ldots, k\}, \\
2 V_{\nu} \beta^{k} \lambda a_{0, k}^{y}-\Omega_{k}^{I S, a}+\kappa \Omega_{0, k}^{P C, a} & =0 \text { for } k \geq 0, \\
2 V_{\nu} \beta^{k} \lambda a_{1, k}^{y}+\Omega_{k-1}^{I S, a}+\kappa \Omega_{1, k}^{P C, a} & =0 \text { for } k \geq 1, \\
2 V_{\nu} \beta^{k} \lambda a_{j, k}^{y}+\kappa \Omega_{j, k}^{P C, a} & =0 \text { for } k \geq 2 \text { and } j \in\{2, \ldots, k\},
\end{aligned}
$$

where $V_{\nu}$ denotes the variance of $\nu_{t}$. After some algebra to get rid of the Lagrange multipliers, I obtain that these first-order conditions amount to the following conditions on coefficients $\left(a_{j, k}^{\pi}, a_{j, k}^{y}\right)_{k \geq 0,0 \leq j \leq k}$ :

$$
\begin{gathered}
\beta \lambda a_{1, k}^{y}+(1+\kappa \sigma) \lambda a_{0, k-1}^{y}+\beta \kappa a_{1, k}^{\pi}+(1+\beta+\kappa \lambda \sigma) \kappa a_{0, k-1}^{\pi}=0 \text { for } k \geq 1 \\
\beta \kappa a_{2, k}^{\pi}+\beta \lambda a_{2, k}^{y}+\beta \kappa a_{1, k-1}^{\pi}+\kappa \lambda \sigma a_{0, k-2}^{y}+(\beta+\kappa \lambda \sigma) \kappa a_{0, k-2}^{\pi}=0 \text { for } k \geq 2 \\
\kappa a_{j, k}^{\pi}+\lambda a_{j, k}^{y}-\lambda a_{j-1, k-1}^{y}=0 \text { for } k \geq 3 \text { and } j \in\{3, \ldots, k\}
\end{gathered}
$$

Noting $v \equiv k-j, A_{j, v}^{\pi} \equiv a_{j, k}^{\pi}$, and $A_{j, v}^{y} \equiv a_{j, k}^{y}$ for $k \geq 0$ and $0 \leq j \leq k$, I can rewrite (A.5), (A.6), (A.10), (A.11) and (A.12) respectively as

$$
\begin{aligned}
A_{0, v}^{y}-A_{1, v}^{y}-\sigma A_{1, v}^{\pi}-(1-\phi) & =0 \text { for } v \geq 0, \\
A_{j, v}^{\pi}-\beta A_{j+1, v}^{\pi}-\kappa A_{j, v}^{y} & =0 \text { for } j \geq 0 \text { and } v \geq 0, \\
\beta \lambda A_{1, v}^{y}+(1+\kappa \sigma) \lambda A_{0, v}^{y}+\beta \kappa A_{1, v}^{\pi}+(1+\beta+\kappa \lambda \sigma) \kappa A_{0, v}^{\pi} & =0 \text { for } v \geq 0, \\
\beta \kappa A_{2, v}^{\pi}+\beta \lambda A_{2, v}^{y}+\beta \kappa A_{1, v}^{\pi}+\kappa \lambda \sigma A_{0, v}^{y}+(\beta+\kappa \lambda \sigma) \kappa A_{0, v}^{\pi} & =0 \text { for } v \geq 0, \\
\kappa A_{j, v}^{\pi}+\lambda A_{j, v}^{y}-\lambda A_{j-1, v}^{y} & =0 \text { for } j \geq 3 \text { and } v \geq 0,
\end{aligned}
$$

which implies that $\forall j \geq 0, A_{j, v}^{\pi}$ and $A_{j, v}^{y}$ do not depend on $v$, so that I can note them $a_{j}^{\pi}$ and $a_{j}^{y}$ respectively. Equations (A.14) and (A.17) imply the recurrence equation $\beta \lambda a_{j+2}^{\pi}-\left(\beta \lambda+\kappa^{2}+\lambda\right) a_{j+1}^{\pi}+$ $\lambda a_{j}^{\pi}=0$ for $j \geq 2$. The roots of the corresponding characteristic polynomial are the following two strictly positive real numbers:

$$
\begin{aligned}
\mu & \equiv \frac{\left(\beta \lambda+\kappa^{2}+\lambda\right)-\sqrt{\left(\beta \lambda+\kappa^{2}+\lambda\right)^{2}-4 \beta \lambda^{2}}}{2 \beta \lambda}<1 \\
\mu^{\prime} & \equiv \frac{\left(\beta \lambda+\kappa^{2}+\lambda\right)+\sqrt{\left(\beta \lambda+\kappa^{2}+\lambda\right)^{2}-4 \beta \lambda^{2}}}{2 \beta \lambda}>1 .
\end{aligned}
$$

Since $\beta \mu^{\prime 2} \geq 1$, as can be readily checked, the solution of the recurrence equation that minimizes $L_{t_{0}}$ is of the form $a_{j}^{\pi}=a_{2}^{\pi} \mu^{j-2}$ for $j \geq 2$. Equation (A.14) then implies that $a_{j}^{y}=\frac{(1-\beta \mu) a_{2}^{\pi}}{\kappa} \mu^{j-2}$ for $j \geq 2$. Coefficients $a_{0}^{\pi}, a_{1}^{\pi}, a_{2}^{\pi}, a_{0}^{y}$ and $a_{1}^{y}$ are then determined by the linear system made of (A.13), (A.14) for $j \in\{0,1\},(\mathrm{A} .15),(\mathrm{A} .16)$ and $a_{2}^{y}=\frac{(1-\beta \mu) a_{2}^{\pi}}{\kappa}$. I thus eventually obtain $a_{0}^{\pi}=a_{0}$, 
$a_{1}^{\pi}=a_{1}, a_{j}^{\pi}=a_{2} \mu^{j-2}$ for $j \geq 2, a_{0}^{y}=\frac{a_{0}-\beta a_{1}}{\kappa}, a_{1}^{y}=\frac{a_{1}-\beta a_{2}}{\kappa}$ and $a_{j}^{y}=\frac{(1-\beta \mu) a_{2}}{\kappa} \mu^{j-2}$ for $j \geq 2$, where $\left[\begin{array}{lll}a_{0} & a_{1} & a_{2}\end{array}\right]^{\prime} \equiv \mathbf{M}^{-1}\left[\begin{array}{ccc}0 & 0 & \kappa(1-\phi)\end{array}\right]^{\prime}$ with

$$
\mathbf{M} \equiv\left[\begin{array}{ccc}
-\left(\beta \kappa^{2}+\kappa \lambda \sigma+\kappa^{2}+\kappa^{3} \lambda \sigma+\lambda\right) & -\beta \kappa(\kappa-\lambda \sigma) & \beta^{2} \lambda \\
\left(\beta \kappa+\kappa^{2} \lambda \sigma+\lambda \sigma\right) \kappa & \beta \kappa(\kappa-\lambda \sigma) & \beta\left(-\beta \lambda \mu+\kappa^{2}+\lambda\right) \\
1 & -(1+\beta+\kappa \sigma) & \beta
\end{array}\right] .
$$

Step 3: let me now turn to the determination of coefficients $\left(b_{j, k}^{\pi}, b_{j, k}^{y}\right)_{k \geq 0,0 \leq j \leq k}$. The first-order conditions of the Lagrangian's minimization with respect to these coefficients are the same as those with respect to coefficients $\left(a_{j, k}^{\pi}, a_{j, k}^{y}\right)_{k \geq 0,0 \leq j \leq k}$ except that $\forall k \geq 0, \forall j \in\{0, \ldots, k\}, a_{j, k}^{\pi}, a_{j, k}^{y}, \Omega_{k}^{I S, a}$ and $\Omega_{j, k}^{P C, a}$ should be respectively replaced by $b_{j, k}^{\pi}, b_{j, k}^{y}, \Omega_{k}^{I S, b}$ and $\Omega_{j, k}^{P C, b}$, and $V_{\nu}$ by $V_{u}$, where the latter denotes the variance of $u_{t}$. Therefore, after some algebra to get rid of the Lagrange multipliers, I obtain that these first-order conditions amount to three conditions on coefficients $\left(b_{j, k}^{\pi}, b_{j, k}^{y}\right)_{k \geq 0,0 \leq j \leq k}$ that are the same as Equations (A.10), (A.11) and (A.12), except that $\forall k \geq 0, \forall j \in\{0, \ldots, k\}, a_{j, k}^{\pi}$ and $a_{j, k}^{y}$ should be respectively replaced by $b_{j, k}^{\pi}$ and $b_{j, k}^{y}$. Noting $v \equiv k-j, B_{j, v}^{\pi} \equiv b_{j, k}^{\pi}$ and $B_{j, v}^{y} \equiv b_{j, k}^{y}$ for $k \geq 0$ and $0 \leq j \leq k$, I can rewrite (A.7), (A.8), (A.9) and these three conditions as

$$
\begin{aligned}
B_{0, v}^{y}-B_{1, v}^{y}-\sigma B_{1, v}^{\pi} & =0 \text { for } v \geq 0, \\
B_{0, v}^{\pi}-\beta B_{1, v}^{\pi}-\kappa B_{0, v}^{y}-1 & =0 \text { for } v \geq 0, \\
B_{j, v}^{\pi}-\beta B_{j+1, v}^{\pi}-\kappa B_{j, v}^{y} & =0 \text { for } j \geq 1 \text { and } v \geq 0, \\
\beta \lambda B_{1, v}^{y}+(1+\kappa \sigma) \lambda B_{0, v}^{y}+\beta \kappa B_{1, v}^{\pi}+(1+\beta+\kappa \lambda \sigma) \kappa B_{0, v}^{\pi} & =0 \text { for } v \geq 0, \\
\beta \kappa B_{2, v}^{\pi}+\beta \lambda B_{2, v}^{y}+\beta \kappa B_{1, v}^{\pi}+\kappa \lambda \sigma B_{0, v}^{y}+(\beta+\kappa \lambda \sigma) \kappa B_{0, v}^{\pi} & =0 \text { for } v \geq 0, \\
\kappa B_{j, v}^{\pi}+\lambda B_{j, v}^{y}-\lambda B_{j-1, v}^{y} & =0 \text { for } j \geq 3 \text { and } v \geq 0,
\end{aligned}
$$

which implies that $\forall j \geq 0, B_{j, v}^{\pi}$ and $B_{j, v}^{y}$ do not depend on $v$, so that I can note them $b_{j}^{\pi}$ and $b_{j}^{y}$ respectively. Equations (A.20) and (A.23) imply the same recurrence equation in $b_{j}^{\pi}$ for $j \geq 2$ as the recurrence equation in $a_{j}^{\pi}$ for $j \geq 2$ obtained above, namely, $\beta \lambda b_{j+2}^{\pi}-\left(\beta \lambda+\kappa^{2}+\lambda\right) b_{j+1}^{\pi}+\lambda b_{j}^{\pi}=0$ for $j \geq 2$. Therefore, in a similar way as above, I get $b_{j}^{\pi}=b_{2}^{\pi} \mu^{j-2}$ for $j \geq 2$. Equation (A.20) then implies that $b_{j}^{y}=\frac{(1-\beta \mu) b_{2}^{\pi}}{\kappa} \mu^{j-2}$ for $j \geq 2$. Coefficients $b_{0}^{\pi}, b_{1}^{\pi}, b_{2}^{\pi}, b_{0}^{y}$ and $b_{1}^{y}$ are then determined by the linear system made of (A.18), (A.19), (A.20) for $j=1,(\mathrm{~A} .21),(\mathrm{A} .22)$ and $b_{2}^{y}=\frac{(1-\beta \mu) b_{2}^{\pi}}{\kappa}$. I thus eventually obtain $b_{0}^{\pi}=b_{0}, b_{1}^{\pi}=b_{1}, b_{j}^{\pi}=b_{2} \mu^{j-2}$ for $j \geq 2, b_{0}^{y}=\frac{b_{0}-\beta b_{1}-1}{\kappa}, b_{1}^{y}=\frac{b_{1}-\beta b_{2}}{\kappa}$ and $b_{j}^{y}=\frac{(1-\beta \mu) b_{2}}{\kappa} \mu^{j-2}$ for $j \geq 2$, where $\left[\begin{array}{lll}b_{0} & b_{1} & b_{2}\end{array}\right]^{\prime} \equiv \mathbf{M}^{-1}\left[\begin{array}{lll}-\lambda(1+\kappa \sigma) & \kappa \lambda \sigma & 1\end{array}\right]^{\prime}$.

Step 4: the coefficients $\left(a_{j, k}^{\pi}, b_{j, k}^{\pi}, a_{j, k}^{y}, b_{j, k}^{y}\right)_{k \geq 0,0 \leq j \leq k}$ that I have obtained in Steps 2 and 3 give me the inflation rate and the output level on the date- $t_{0}$ Ramsey-optimal feasible path as functions of shocks having occurred since date $t_{0}$. By making $t_{0}$ tend towards $-\infty$, I straightforwardly get these two variables on the timeless-perspective Ramsey-optimal feasible path, as functions of all current and past shocks:

$$
\begin{aligned}
{\left[\begin{array}{l}
\pi_{t} \\
y_{t}
\end{array}\right] } & =\left[\begin{array}{cc}
a_{0} & b_{0} \\
\frac{\kappa \phi+a_{0}-\beta a_{1}}{\kappa} & \frac{b_{0}-\beta b_{1}-1}{\kappa}
\end{array}\right]\left[\begin{array}{c}
\nu_{t} \\
u_{t}
\end{array}\right]+\left[\begin{array}{cc}
a_{1} & b_{1} \\
\frac{a_{1}-\beta a_{2}}{\kappa} & \frac{b_{1}-\beta b_{2}}{\kappa}
\end{array}\right]\left[\begin{array}{c}
\nu_{t-1} \\
u_{t-1}
\end{array}\right] \\
& +\sum_{j=2}^{+\infty}\left[\begin{array}{cc}
a_{2} \mu^{j-2} & b_{2} \mu^{j-2} \\
\frac{(1-\beta \mu) a_{2} \mu^{j-2}}{\kappa} & \frac{(1-\beta \mu) b_{2} \mu^{j-2}}{\kappa}
\end{array}\right]\left[\begin{array}{c}
\nu_{t-j} \\
u_{t-j}
\end{array}\right] .
\end{aligned}
$$

Using this result and the IS equation (1), I then residually obtain the interest rate on the timelessperspective Ramsey-optimal feasible path, as a function of all past shocks:

$$
\begin{aligned}
i_{t}= & {\left[\begin{array}{ll}
\frac{(1+\beta-\beta \mu+\kappa \sigma) a_{2}-a_{1}}{\kappa \sigma} & \frac{(1+\beta-\beta \mu+\kappa \sigma) b_{2}-b_{1}}{\kappa \sigma}
\end{array}\right]\left[\begin{array}{c}
\nu_{t-1} \\
u_{t-1}
\end{array}\right] } \\
& +\sum_{j=2}^{+\infty}\left[\begin{array}{ll}
\frac{-(\kappa-\lambda \sigma) a_{2} \mu^{j-1}}{\lambda \sigma} & \frac{-(\kappa-\lambda \sigma) b_{2} \mu^{j-1}}{\lambda \sigma}
\end{array}\right]\left[\begin{array}{c}
\nu_{t-j} \\
u_{t-j}
\end{array}\right] .
\end{aligned}
$$


Finally, using $\beta \lambda \mu^{2}-\left(\lambda+\beta \lambda+\kappa^{2}\right) \mu+\lambda=0$, I can easily rewrite the system made of (A.24) and (A.25) in the VARMA form stated in Proposition 3, where

$$
\begin{aligned}
& \boldsymbol{\Omega}_{0} \equiv\left[\begin{array}{cc}
a_{0} & b_{0} \\
\frac{\kappa \phi+a_{0}-\beta a_{1}}{\kappa} & \frac{b_{0}-\beta b_{1}-1}{\kappa} \\
\hline 0 & 0
\end{array}\right], \boldsymbol{\Omega}_{2} \equiv\left[\begin{array}{cc}
\frac{\lambda(1-\mu)\left(a_{2}-\mu a_{1}\right)}{\kappa^{2} \mu} & \frac{\lambda(1-\mu)\left(b_{2}-\mu b_{1}\right)}{\kappa^{2} \mu} \\
\frac{a_{2}-\mu a_{1}}{\kappa} & \frac{b_{2}-\mu b_{1}}{\kappa} \\
\frac{-(\kappa-\lambda \sigma)(1-\mu)\left(a_{2}-\mu a_{1}\right)}{\kappa^{2} \sigma} & \frac{-(\kappa-\lambda \sigma)(1-\mu)\left(b_{2}-\mu b_{1}\right)}{\kappa^{2} \sigma}
\end{array}\right] \text { and } \boldsymbol{\Omega}_{1} \equiv \\
& {\left[\begin{array}{cc}
\frac{\lambda(1-\mu)\left(\kappa \phi \mu+a_{1}-\mu a_{0}\right)}{\kappa^{2} \mu}-\beta\left(a_{2}-\mu a_{1}\right) & \frac{\lambda(1-\mu)\left(b_{1}-\mu b_{0}+\mu\right)}{\kappa^{2} \mu} \\
\frac{\left(\kappa \phi \mu+a_{1}-\mu a_{0}\right)-\beta\left(b_{2}-\mu b_{1}\right)}{\kappa} & \frac{\left(b_{1}-\mu b_{0}+\mu\right)-\beta\left(b_{2}\right)}{\kappa^{2} \sigma} \\
\frac{\left.-(\kappa-\lambda \sigma)(1-\mu)\left(\kappa \phi \mu+a_{1}-\mu a^{2}\right)+\beta \mu+\kappa \sigma\right)\left(a_{2}-\mu a_{1}\right)}{\kappa^{2} \sigma} & \frac{-(\kappa-\lambda \sigma)(1-\mu)\left(b_{1}-\mu b_{0}+\mu\right)+\kappa(1+\beta-\beta \mu+\kappa \sigma)\left(b_{2}-\mu b_{1}\right)}{\kappa^{2} \sigma}
\end{array}\right] .}
\end{aligned}
$$

Proposition 3 follows.

\section{A.3 Proof of Proposition 4}

Consider an interest-rate rule of type

$$
i_{t}=a \pi_{t-1}+b y_{t-1}+c \nu_{t-1}+d u_{t-1}+e \nu_{t-2}+f u_{t-2}
$$

where $(a, b, c, d, e, f) \in \mathbb{R}^{6}$. I proceed in three steps, along the lines of Appendix A.1.

Step 1: identical to Step 1 of Appendix A.1, except that (i) (A.1) should be replaced by (A.26), and (ii) the third element of the last term in (A.2) should be $-\left(1+\frac{\kappa \phi \sigma}{\beta}\right) \nu_{t}+\frac{\sigma}{\beta} u_{t}+\sigma c \nu_{t-1}+\sigma d u_{t-1}+\sigma e \nu_{t-2}+\sigma f u_{t-2}$ instead of $\Phi_{t}$.

Step 2: by construction, (1) and (2) are consistent with (5). Moreover, since $\nu_{t}$ and $u_{t}$ are independent of each other, I easily get, after some algebra to re-arrange the terms, that (A.26) is consistent with the path (5) if and only if

$$
b=\frac{-\lambda(1-\mu)}{\kappa \mu} a-\frac{(\kappa-\lambda \sigma) \mu(1-\mu)}{\kappa \sigma} \text { and }\left[\begin{array}{l}
c \\
d \\
e \\
f
\end{array}\right]=\left[\begin{array}{l}
\boldsymbol{\Omega}_{0}^{\prime} \\
\boldsymbol{\Omega}_{1}^{\prime}
\end{array}\right]\left[\begin{array}{c}
-a \\
\frac{\lambda(1-\mu)}{\kappa \mu} a \\
0
\end{array}\right]+\left[\begin{array}{l}
\boldsymbol{\Omega}_{1}^{\prime} \\
\boldsymbol{\Omega}_{2}^{\prime}
\end{array}\right]\left[\begin{array}{l}
0 \\
0 \\
1
\end{array}\right]
$$

Step 3: identical to Step 3 of Appendix A.1, except that (4), (A.1) and (A.4) should be respectively replaced by (5), (A.26) and (A.27). Proposition 4 follows.

\section{A.4 Proof of Proposition 6}

I proceed in two steps: first, I determine the unique $\left(\alpha_{j}^{\pi}, \alpha_{j}^{y}, \alpha_{j}^{i}\right)_{1 \leq j \leq 2} \in \mathbb{R}^{6}$ such that the interest-rate rule $i_{t}=\sum_{j=1}^{2}\left(\alpha_{j}^{\pi} \pi_{t-j}+\alpha_{j}^{y} y_{t-j}+\alpha_{j}^{i} i_{t-j}\right)$ is satisfied on the path (5); second, I show that the set of interest-rate rules that (i) involve only past actions, and (ii) are satisfied on the path (5), is the set $\left\{K(L)\left[i_{t}-\sum_{j=1}^{2}\left(\alpha_{j}^{\pi} \pi_{t-j}+\alpha_{j}^{y} y_{t-j}+\alpha_{j}^{i} i_{t-j}\right)\right] \mid K(L) \in \mathbb{R}[L]\right\}$, where $L$ denotes the lag operator and $\mathbb{R}[L]$ the set of polynomials in $L$ with real-number coefficients, and that either all the rules of this set ensure local equilibrium determinacy, or none of them does.

Step 1: the path (5) can be equivalently rewritten as (A.24) and (A.25). Define implicitly the $2 \times 2$ matrices $\mathbf{P}_{0}, \mathbf{P}_{1}$, and $\mathbf{P}_{2}$ by rewriting (A.24) as

$$
\left[\begin{array}{l}
\pi_{t} \\
y_{t}
\end{array}\right]=\mathbf{P}_{0}\left[\begin{array}{l}
\nu_{t} \\
u_{t}
\end{array}\right]+\mathbf{P}_{1}\left[\begin{array}{l}
\nu_{t-1} \\
u_{t-1}
\end{array}\right]+\mathbf{P}_{2} \sum_{j=2}^{+\infty} \mu^{j-2}\left[\begin{array}{c}
\nu_{t-j} \\
u_{t-j}
\end{array}\right]
$$


and the $1 \times 2$ matrices $\mathbf{Q}_{1}$ and $\mathbf{R}_{1}$ by rewriting (A.25) as

$$
i_{t}=\mathbf{Q}_{1}\left[\begin{array}{c}
\nu_{t-1} \\
u_{t-1}
\end{array}\right]+\mathbf{R}_{1} \sum_{j=2}^{+\infty} \mu^{j-2}\left[\begin{array}{c}
\nu_{t-j} \\
u_{t-j}
\end{array}\right]
$$

By recurrence on $k \in\{1, \ldots, 2\}$, using (A.28) and (A.29) at date $t-k$, I easily get that, on the path (5), for any $k \in\{0,1,2\}$ and any $\left(\alpha_{1}^{i}, \alpha_{2}^{i}\right) \in \mathbb{R}^{2}$,

$$
\begin{aligned}
& i_{t}=\mathbb{1}_{k \geq 1} \sum_{j=1}^{k}\left(\mathbf{Q}_{j} \mathbf{P}_{0}^{-1}\left[\begin{array}{ll}
\pi_{t-j} & y_{t-j}
\end{array}\right]+\alpha_{j}^{i} i_{t-j}\right)+\mathbf{Q}_{k+1}\left[\begin{array}{c}
\nu_{t-k-1} \\
u_{t-k-1}
\end{array}\right]+\mathbf{R}_{k+1} \sum_{j=k+2}^{+\infty} \mu^{j-k-2}\left[\begin{array}{c}
\nu_{t-j} \\
u_{t-j}
\end{array}\right]
\end{aligned}
$$

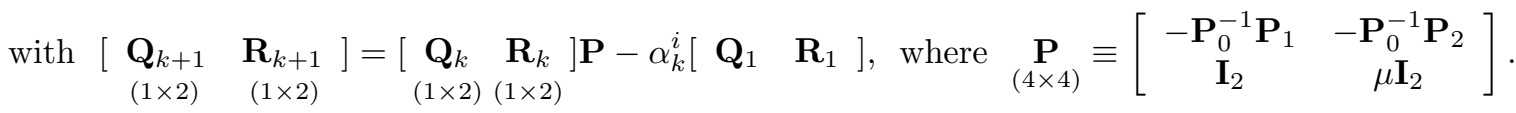

Since $\left[\begin{array}{ll}\mathbf{Q}_{3} & \mathbf{R}_{3}\end{array}\right]=\left[\begin{array}{ll}\mathbf{Q}_{1} & \mathbf{R}_{1}\end{array}\right]\left(\mathbf{P}^{2}-\alpha_{1}^{i} \mathbf{P}-\alpha_{2}^{i} \mathbf{I}_{4}\right)$, the choice of

$$
\left[\begin{array}{ll}
\alpha_{1}^{i} & \alpha_{2}^{i}
\end{array}\right]=\left[\begin{array}{ll}
\mathbf{Q}_{1} & \mathbf{R}_{1}
\end{array}\right] \mathbf{P}^{2} \mathbf{J}\left[\begin{array}{c}
{\left[\begin{array}{cc}
\mathbf{Q}_{1} & \mathbf{R}_{1}
\end{array}\right] \mathbf{P J}} \\
{\left[\begin{array}{ll}
\mathbf{Q}_{1} & \mathbf{R}_{1}
\end{array}\right] \mathbf{J}}
\end{array}\right]^{-1}, \text { where } \mathbf{J}=\left[\begin{array}{llll}
1 & 0 & 0 & 0 \\
0 & 1 & 0 & 0
\end{array}\right]^{\prime}
$$

implies $\mathbf{Q}_{3}=\mathbf{0}$. Moreover, it can be analytically checked, with the help of a symbolic-computation software, that this choice also implies $\mathbf{R}_{3}=\mathbf{0}$. Therefore, there exists a unique $\left(\alpha_{j}^{\pi}, \alpha_{j}^{y}, \alpha_{j}^{i}\right)_{1 \leq j \leq 2} \in \mathbb{R}^{6}$ such that

$$
i_{t}=\sum_{j=1}^{2}\left(\alpha_{j}^{\pi} \pi_{t-j}+\alpha_{j}^{y} y_{t-j}+\alpha_{j}^{i} i_{t-j}\right)
$$

is satisfied on the path (5), and it is characterized by $\left[\begin{array}{cc}\alpha_{k}^{\pi} & \alpha_{k}^{y}\end{array}\right]=\mathbf{Q}_{k} \mathbf{P}_{0}^{-1}$ for $k \in\{1,2\}$ and (A.30). This result implies in particular that when $O_{t}^{\mathcal{C B}}=\left\{\pi^{t-1}, y^{t-1}, i^{t-1}\right\}$, the timeless-perspective Ramseyoptimal feasible path is the path (5).

Step 2: (b) trivially implies (a). To show that (a) implies (b), note first that $\boldsymbol{\Omega}_{0}$ (defined in Step 4 of Appendix A.2) is of rank 2 (over $\mathbb{R}$ ). Therefore, the system made of the first two lines of the path (5), taken at all dates $t \in \mathbb{Z}$, implies no equation without exogenous shocks. As a consequence, any interest-rate rule of type (3) that is (i) such that $\forall(j, k) \in\left\{1, \ldots, n^{e}\right\}^{2}, e_{j, k}^{\pi}=e_{j, k}^{y}=e_{j, k}^{i}=0$, (ii) such that $\forall k \in\left\{1, \ldots, n^{h}\right\}, h_{k}^{\nu}=h_{k}^{u}=0$, and (iii) consistent with the path (5), is necessarily of type

$$
K(L)\left[i_{t}-\sum_{j=1}^{2}\left(\alpha_{j}^{\pi} \pi_{t-j}+\alpha_{j}^{y} y_{t-j}+\alpha_{j}^{i} i_{t-j}\right)\right]=0
$$

with $K(L) \equiv \sum_{j=0}^{n^{k}} k_{j} L^{j}$, where $L$ denotes the lag operator, $n^{k} \in \mathbb{N}$, all $k_{j}$ are real numbers and, without any loss in generality, $k_{0}$ is normalized to one. Now, for any such $K(L)$, (A.32) has a unique stationary solution in $\left[i_{t}-\sum_{j=1}^{2}\left(\alpha_{j}^{\pi} \pi_{t-j}+\alpha_{j}^{y} y_{t-j}+\alpha_{j}^{i} i_{t-j}\right)\right]_{t \in \mathbb{Z}}$, namely $i_{t}-\sum_{j=1}^{2}\left(\alpha_{j}^{\pi} \pi_{t-j}+\alpha_{j}^{y} y_{t-j}+\alpha_{j}^{i} i_{t-j}\right)=0$, which corresponds to the interest-rate rule (A.31). Therefore, for any such $K(L)$, the system made of the IS equation (1), the Phillips curve (2), and the interest-rate rule (A.32) has exactly the same stationary solutions in $\left(\pi_{t}, y_{t}, i_{t}\right)_{t \in \mathbb{Z}}$ as the system made of the IS equation (1), the Phillips curve (2), and the interest-rate rule (A.31). Proposition 6 follows.

\section{A.5 Proof of Proposition 11}

Svensson and Woodford (2005) provide the following expressions for $\mathbb{E}_{t}\left\{\pi_{t+1}\right\}, \mathbb{E}_{t}\left\{x_{t+1}\right\}$, and $i_{t}$, as functions of $r_{t}^{n}$ and $u^{t}$, on the timeless-perspective Ramsey-optimal feasible path (when $\mathcal{C B}$ 's observation 
set is such that $\mathcal{C B}$ 's observation constraint is slack in the optimization programme defining this path): ${ }^{65}$

$$
\begin{gathered}
\mathbb{E}_{t}\left\{\pi_{t+1}\right\}=\frac{\rho \mu}{1-\beta \rho \mu} u_{t}-\frac{(1-\mu) \rho \mu}{1-\beta \rho \mu} \sum_{j=1}^{+\infty} \mu^{j-1} u_{t-j}, \\
\mathbb{E}_{t}\left\{x_{t+1}\right\}=\frac{-\kappa \rho \mu}{\lambda(1-\beta \rho \mu)} \sum_{j=0}^{+\infty} \mu^{j} u_{t-j}, \\
i_{t}=\omega r_{t-1}^{n}+\frac{(\lambda \sigma-\kappa) \rho^{2} \mu}{\lambda \sigma(1-\beta \rho \mu)} u_{t-1}-\frac{(\lambda \sigma-\kappa)(1-\mu) \rho \mu}{\lambda \sigma(1-\beta \rho \mu)} \sum_{j=1}^{+\infty} \mu^{j-1} u_{t-j} .
\end{gathered}
$$

Using these expressions, the IS equation (25), the Phillips curve (26), $x_{t}=y_{t}-\phi \nu_{t}$, and $\nu_{t}=\frac{\sigma}{1-\phi} r_{t}^{n}+$ $\frac{\sigma \omega^{2}}{(1-\phi)(1-\omega)} r_{t-1}^{n}$, I easily get $\pi_{t}$ and $y_{t}$ on this path as functions of $r_{t}^{n}, r_{t-1}^{n}$, and $u^{t}$ :

$$
\begin{aligned}
& \pi_{t}=u_{t}+\left(\frac{\rho \mu}{1-\beta \rho \mu}-\rho\right) u_{t-1}-\frac{(1-\mu) \rho \mu}{1-\beta \rho \mu} \sum_{j=2}^{+\infty} \mu^{j-2} u_{t-j} \\
& y_{t}=\frac{\sigma}{1-\phi} r_{t}^{n}-\frac{(1-\phi-\omega) \sigma \omega}{(1-\phi)(1-\omega)} r_{t-1}^{n}-\frac{\kappa \rho \mu}{\lambda(1-\beta \rho \mu)} \sum_{j=1}^{+\infty} \mu^{j-1} u_{t-j} .
\end{aligned}
$$

Multiplying the left- and right-hand sides of (A.36) by $(1-\rho L)(1-\mu L)$, and the left- and right-hand sides of (A.37) by $(1-\rho L)(1-\omega L)(1-\mu L)$, leads to the first two lines of (31). Now replace $r_{t-1}^{n}$ and $r_{t-2}^{n}$, in (A.37) taken at date $t-1$, by their expressions in (A.35) taken at dates $t$ and $t-1$ respectively. Then replace $u_{t-1}$, in the resulting equation, by its expression in (A.36) taken at date $t-1$. Finally, multiply the left- and right-hand sides of the resulting equation by $\frac{(1-\phi) \omega}{\sigma}$, and thus get

$$
z_{t}=q_{2} u_{t-2}+r_{2} \sum_{j=3}^{+\infty} \mu^{j-3} u_{t-j}
$$

where $z_{t} \equiv i_{t}-\frac{(1-\phi-\omega) \omega}{1-\omega} i_{t-1}-\frac{(1-\phi) \omega}{\sigma} y_{t-1}+\frac{(\lambda \sigma-\kappa)(1-\rho-\mu) \rho \mu}{\lambda \sigma(1-\beta \rho \mu)} \pi_{t-1}$,

$$
\begin{aligned}
q_{2} & \equiv \frac{(\lambda \sigma-\kappa) \rho \mu}{\lambda \sigma(1-\beta \rho \mu)}\left\{(1-\rho-\mu)\left[\frac{(1-\phi-\omega) \omega}{1-\omega}+\frac{\rho \mu}{1-\beta \rho \mu}-\rho\right]-(1-\mu) \mu\right\}+\frac{\kappa(1-\phi) \rho \omega \mu}{\lambda \sigma(1-\beta \rho \mu)}, \\
r_{2} & \equiv \frac{(\lambda \sigma-\kappa)(1-\mu) \rho \mu^{2}}{\lambda \sigma(1-\beta \rho \mu)}\left[\frac{(1-\phi-\omega) \omega}{1-\omega}-\frac{(1-\rho-\mu) \rho}{1-\beta \rho \mu}-\mu\right]+\frac{\kappa(1-\phi) \rho \omega \mu^{2}}{\lambda \sigma(1-\beta \rho \mu)} .
\end{aligned}
$$

Let $p_{1} \equiv \frac{\rho \mu}{1-\beta \rho \mu}-\rho$ and $p_{2} \equiv \frac{-(1-\mu) \rho \mu}{1-\beta \rho \mu}$, so that (A.36) is written

$$
\pi_{t}=u_{t}+p_{1} u_{t-1}+p_{2} \sum_{j=2}^{+\infty} \mu^{j-2} u_{t-j} .
$$

Replace $u_{t-2}$ in (A.38) by its expression in (A.39) taken at date $t-2$, and subtract the left- and righthand sides of the resulting equation by, respectively, the left- and right-hand sides of (A.38) taken at date $t-1$ and multiplied by an arbitrary real number $\alpha_{1}^{z}$, to get

$$
z_{t}=\alpha_{1}^{z} z_{t-1}+q_{2} \pi_{t-2}+q_{3}\left(\alpha_{1}^{z}\right) u_{t-3}+r_{3}\left(\alpha_{1}^{z}\right) \sum_{j=4}^{+\infty} \mu^{j-4} u_{t-j},
$$

where $q_{3}\left(\alpha_{1}^{z}\right) \equiv-q_{2} \alpha_{1}^{z}+r_{2}-p_{1} q_{2}$ and $r_{3}\left(\alpha_{1}^{z}\right) \equiv-r_{2} \alpha_{1}^{z}+r_{2} \mu-p_{2} q_{2}$. Similarly, replace $u_{t-3}$ in (A.40) by its expression in (A.39) taken at date $t-3$, and subtract the left- and right-hand sides of the resulting equation by, respectively, the left- and right-hand sides of (A.38) taken at date $t-2$ and multiplied by an arbitrary real number $\alpha_{2}^{z}$, to get

$$
z_{t}=\alpha_{1}^{z} z_{t-1}+q_{2} \pi_{t-2}+\alpha_{2}^{z} z_{t-2}+q_{3}\left(\alpha_{1}^{z}\right) \pi_{t-3}+q_{4}\left(\alpha_{1}^{z}, \alpha_{2}^{z}\right) u_{t-4}+r_{4}\left(\alpha_{1}^{z}, \alpha_{2}^{z}\right) \sum_{j=5}^{+\infty} \mu^{j-5} u_{t-j},
$$

${ }^{65}$ There are three differences between Equations (A.33), (A.34), (A.35), and Equations (26), (27), (32) in Svensson and Woodford (2005). First, as already explained in Footnote 47, I have set $\bar{r}=0$ for simplicity. Second, for reasons of consistency with Section 2's notations, I use the notation $\mu$ instead of $c$. Third, I have corrected a typo in Equation (27), i.e. removed the negative sign just after the equality sign. 
where $q_{4}\left(\alpha_{1}^{z}, \alpha_{2}^{z}\right) \equiv-q_{2} \alpha_{2}^{z}+r_{3}\left(\alpha_{1}^{z}\right)-p_{1} q_{3}\left(\alpha_{1}^{z}\right)$ and $r_{4}\left(\alpha_{1}^{z}, \alpha_{2}^{z}\right) \equiv-r_{2} \alpha_{2}^{z}+r_{3}\left(\alpha_{1}^{z}\right) \mu-p_{2} q_{3}\left(\alpha_{1}^{z}\right)$. The choice of

$$
\left[\begin{array}{c}
\alpha_{1}^{z} \\
\alpha_{2}^{z}
\end{array}\right]=\left[\begin{array}{cc}
r_{2}-p_{1} q_{2} & q_{2} \\
r_{2} \mu-p_{2} q_{2} & r_{2}
\end{array}\right]^{-1}\left[\begin{array}{c}
\left(p_{1}^{2}-p_{2}\right) q_{2}+\left(\mu-p_{1}\right) r_{2} \\
\left(p_{1}-\mu\right) p_{2} q_{2}+\left(\mu^{2}-p_{2}\right) r_{2}
\end{array}\right]
$$

implies $q_{4}\left(\alpha_{1}^{z}, \alpha_{2}^{z}\right)=r_{4}\left(\alpha_{1}^{z}, \alpha_{2}^{z}\right)=0$. Replacing $\alpha_{1}^{z}$ and $\alpha_{2}^{z}$ in (A.41) by their expressions in (A.42) leads to the third line of (31) with

$$
\left[\begin{array}{lll}
\alpha_{1}^{\pi} & \alpha_{1}^{y} & \alpha_{1}^{i} \\
\alpha_{2}^{\pi} & \alpha_{2}^{y} & \alpha_{2}^{i} \\
\alpha_{3}^{\pi} & \alpha_{3}^{y} & \alpha_{3}^{i}
\end{array}\right] \equiv\left[\begin{array}{ccc}
\frac{-(\lambda \sigma-\kappa)(1-\rho-\mu) \rho \mu}{\lambda \sigma(1-\beta \mu)} & \frac{(1-\phi) \omega}{\sigma} & \frac{(1-\phi-\omega) \omega}{1-\omega}+\alpha_{1}^{z} \\
\frac{(\lambda \sigma-\kappa)(1-\rho-\mu) \rho \mu}{\lambda \sigma(1-\beta \rho \mu)} \alpha_{1}^{z}+q_{2} & \frac{-(1-\phi) \omega}{\sigma} \alpha_{1}^{z} & \frac{-(1-\phi-\omega) \omega}{1-\omega} \alpha_{1}^{z}+\alpha_{2}^{z} \\
\frac{(\lambda \sigma-\kappa)(1-\rho-\mu) \rho \mu}{\lambda \sigma(1-\beta \rho \mu)} \alpha_{2}^{z}-q_{2} \alpha_{1}^{z}+r_{2}-\left(\frac{\rho \mu}{1-\beta \rho \mu}-\rho\right) q_{2} & \frac{-(1-\phi) \omega}{\sigma} \alpha_{2}^{z} & \frac{-(1-\phi-\omega) \omega}{1-\omega} \alpha_{2}^{z}
\end{array}\right] .
$$

Therefore, the timeless-perspective Ramsey-optimal feasible path determined by Svensson and Woodford (2005) can be rewritten in the form (31), which is consistent with $O_{t}^{\mathcal{C B}}=\left\{\pi^{t-1}, y^{t-1}, i^{t-1}\right\}$. Now, this path is determined by Svensson and Woodford (2005) under the assumption that $\mathcal{C B}^{\prime}$ 's observation constraint is slack. As a consequence, (31) is the timeless-perspective Ramsey-optimal feasible path when $O_{t}^{\mathcal{C B}}=\left\{\pi^{t-1}, y^{t-1}, i^{t-1}\right\}$. Proposition 11 follows. 